\title{
Stereosective Synthesis of the Antiprotozoal Lactone Passifloricin A and Seven Isomers Thereof
}

Juan Murga, ${ }^{\dagger}$ Jorge García-Fortanet, ${ }^{\dagger}$ Miguel Carda, ${ }^{\dagger}$ and J. Alberto Marco ${ }^{\ddagger}$

‘Departamento de Química Inorgánica y Orgánica, Univ. Jaume I, E-12080 Castellón,

Spain; and "Departamento de Química Orgánica, Univ. de Valencia, E-46100 Burjassot, Valencia, Spain.

\section{Supporting Information}

\section{Contents:}

S-2/S-8: $\quad$ General procedures and synthetic schemes

S-9/S-23: Analytical data of intermediate compounds

S-24/S-47: Analytical data of lactones 1-4 and 18a-d and of some derivatives; NMR spectra of lactones 1-4 and 18a-d 
General features. NMR spectra were measured at $25{ }^{\circ} \mathrm{C}$. The signals of the deuterated solvent $\left(\mathrm{CDCl}_{3}\right)$ were taken as the reference. Multiplicity assignments of ${ }^{13} \mathrm{C}$ signals were made by means of the DEPT pulse sequence. High resolution mass spectra were run by the electron impact mode (EIMS, $70 \mathrm{eV}$ ) or by the FAB mode ( $m$-nitrobenzyl alcohol matrix). IR data were measured with oily films on $\mathrm{NaCl}$ plates (oils) or $\mathrm{KBr}$ pellets (solids) and are given only for molecules with relevant functional groups $(\mathrm{OH}, \mathrm{C}=\mathrm{O})$. Optical rotations were measured at $25^{\circ} \mathrm{C}$. Experiments which required an inert atmosphere were carried out under dry $\mathrm{N}_{2}$ in a flame-dried glassware. $\mathrm{Et}_{2} \mathrm{O}$ and THF were freshly distilled from sodium/benzophenone ketyl and transferred via syringe. Dichloromethane was freshly distilled from $\mathrm{CaH}_{2}$. Tertiary amines were freshly distilled from $\mathrm{KOH}$. Commercially available reagents were used as received. Unless detailed otherwise, "work-up" means pouring the reaction mixture into brine, followed by extraction with the solvent indicated in parenthesis. If the reaction medium was acidic (basic), an additional washing with $5 \%$ aq $\mathrm{NaHCO}_{3}\left(\mathrm{aq} \mathrm{NH}_{4} \mathrm{Cl}\right.$ ) was performed. New washing with brine, drying over anhydrous $\mathrm{Na}_{2} \mathrm{SO}_{4}$ and elimination of the solvent under reduced pressure were followed by chromatography on a silica gel column $(60-200 \mu \mathrm{m})$ with the indicated eluent. Where solutions were filtered through a Celite pad, the pad was additionally washed with the same solvent used, and the washings incorporated to the main organic layer. Acronyms used hereafter: DIP-Cl = diisopinocampheylboron chloride; TBS $=t$-butyldimethylsilyl; TBAF $=$ tetra- $n$-butyl ammonium fluoride hydrate; DMAP $=4-(\mathrm{N}, \mathrm{N}-$ dimethylamino)pyridine; PPTS $=$ pyridinium $p$ toluenesulfonate.

\section{General reaction conditions (see schemes)}

General procedure for asymmetric allylations (reaction $+a$ ). Allylmagnesium bromide (commercial $1 \mathrm{M}$ solution in $\mathrm{Et}_{2} \mathrm{O}, 10 \mathrm{~mL}, 10 \mathrm{mmol}$ ) was added dropwise under $\mathrm{N}_{2}$ via syringe to a solution of $(+)$ DIP-Cl (3.85 g, $12 \mathrm{mmol})$ in dry $\mathrm{Et}_{2} \mathrm{O}(50 \mathrm{~mL})$ cooled in a dry ice-acetone bath. After replacing the latter by an ice bath, the mixture was stirred for $1 \mathrm{~h}$. The solution was then allowed to stand, which caused precipitation of magnesium chloride. The supernatant solution was then carefully transferred to another flask via canula. After cooling this flask at $-100^{\circ} \mathrm{C}$, a solution of the appropriate aldehyde (8 $\mathrm{mmol})$ in dry $\mathrm{Et}_{2} \mathrm{O}(25 \mathrm{~mL})$ was added dropwise via syringe. The resulting solution was further stirred at the same temp. for $1 \mathrm{~h}$. The reaction mixture was then quenched through addition of phosphate $\mathrm{pH} 7$ buffer solution $(50 \mathrm{~mL}), \mathrm{MeOH}(50 \mathrm{~mL})$ and $30 \% \mathrm{H}_{2} \mathrm{O}_{2}(25 \mathrm{~mL})$. After stirring for $30 \mathrm{~min}$., the mixture was poured onto satd. aq $\mathrm{NaHCO}_{3}$ and worked up $\left(\mathrm{Et}_{2} \mathrm{O}\right)$. Column chromatography on silica gel (hexanes-EtOAc mixtures) afforded the desired homoallylic alcohol. Reaction $-a$ is performed in the same way except for the use of (-)-DIP-Cl.

General procedure for sequential ozonolysis/asymmetric allylation (reaction $+b$ or $-b$ ). The appropriate olefin $(10 \mathrm{mmol})$ was dissolved in dry $\mathrm{CH}_{2} \mathrm{Cl}_{2}(100 \mathrm{~mL})$ and cooled to $-78^{\circ} \mathrm{C}$. A stream of ozone-oxygen was bubbled through the solution until persistence of the bluish color. Dry $\mathrm{N}_{2}$ was then bubbled through the solution for $10 \mathrm{~min}$. at the same temperature. After addition of $\operatorname{PPh}_{3}(5.25 \mathrm{~g}, 20$ 
mmol), the solution was left to stir at room temp. for $2 \mathrm{~h}$, then worked up (extraction with $\mathrm{CH}_{2} \mathrm{Cl}_{2}$ ). Solvent removal gave a solid material, which was washed three times with cold pentane (3 x $15 \mathrm{~mL})$. The solid $\left(\mathrm{Ph}_{3} \mathrm{PO}\right)$ was discarded, and the organic phase was evaporated in vacuo to yield a colorless oil which was used as such in the asymmetric allylation (for weight calculations, the yield of the ozonolysis step was assumed to be quantitative). The overall process is described as $+b$ or $-b$ according to the use of (+)- or (-)-DIP-Cl in the allylation step.

General procedure for silylations with TBSCl (reaction c). The appropriate alcohol (8 mmol) was dissolved under $\mathrm{N}_{2}$ in dry DMF $(50 \mathrm{~mL})$ and treated sequentially with imidazole $(817 \mathrm{mg}, 12 \mathrm{mmol})$ and TBSCl $(1.74 \mathrm{~g}, 11.5 \mathrm{mmol})$. The reaction mixture was then stirred for $18 \mathrm{~h}$ at room temp. and worked up (extraction with $\mathrm{Et}_{2} \mathrm{O}$ ). Column chromatography on silica gel (hexanes-EtOAc mixtures) afforded the desired silylated derivative.

General procedure for silylations with TBSOTf (reaction $d$ ). The appropriate alcohol (4 mmol) was dissolved under $\mathrm{N}_{2}$ in dry $\mathrm{CH}_{2} \mathrm{Cl}_{2}(20 \mathrm{~mL})$ and treated sequentially with 2,6-lutidine $(700 \mu \mathrm{L}, 6 \mathrm{mmol})$ and TBSOTf $(1.15 \mathrm{~mL}, 5 \mathrm{mmol})$. The reaction mixture was then stirred for $1 \mathrm{~h}$ at room temp. and worked up (extraction with $\mathrm{CH}_{2} \mathrm{Cl}_{2}$ ). Column chromatography on silica gel (hexanes-EtOAc mixtures) afforded the desired silylated derivative. For silylation of diols, the molar amounts of TBSOTf and 2,6lutidine were doubled.

General procedure for acylations with cinnamoyl chloride (reaction e). The appropriate alcohol ( 0.5 mmol) was dissolved under $\mathrm{N}_{2}$ in dry $\mathrm{CH}_{2} \mathrm{Cl}_{2}(10 \mathrm{~mL})$ and treated sequentially with triethyl amine (700 $\mu \mathrm{L}, 5 \mathrm{mmol}$ ) and DMAP (6 mg, $0.05 \mathrm{mmol})$. Cinnamoyl chloride ( $833 \mathrm{mg}, 5 \mathrm{mmol}$ ) was then added at room temp. under stirring in four portions at intervals of $45 \mathrm{~min}$. After a total stirring time of $3 \mathrm{~h}$, the reaction mixture was worked up (extraction with $\mathrm{CH}_{2} \mathrm{Cl}_{2}$ ). Column chromatography on silica gel (hexanes-EtOAc, 19:1) afforded the desired ester.

General procedure for acylations with acryloyl chloride (reaction $f)$. The appropriate alcohol $(0.5$ mmol) was dissolved under $\mathrm{N}_{2}$ in dry $\mathrm{CH}_{2} \mathrm{Cl}_{2}(20 \mathrm{~mL})$, cooled to $-78^{\circ} \mathrm{C}$ and treated sequentially with ethyl diisopropylamine $(1.3 \mathrm{~mL}, 7.5 \mathrm{mmol})$ and acryloyl chloride $(400 \mu \mathrm{L}, 5 \mathrm{mmol})$. The reaction mixture was stirred for $2 \mathrm{~h}$ at $-78^{\circ} \mathrm{C}$ and then worked up (extraction with $\mathrm{CH}_{2} \mathrm{Cl}_{2}$ ). Column chromatography on silica gel (hexanes-EtOAc, 19:1) afforded the desired ester.

General procedure for ring-closing metathesis with ruthenium catalysts (reaction $g$ ). The appropriate diolefin $(1 \mathrm{mmol})$ was dissolved under $\mathrm{N}_{2}$ in dry, degassed $\mathrm{CH}_{2} \mathrm{Cl}_{2}(100 \mathrm{~mL})$ and treated with the appropriate ruthenium catalyst $(80 \mathrm{mg})$. The mixture was heated at reflux until consumption of the starting material (ca. $3 \mathrm{~h}$, TLC monitoring!). Solvent removal in vacuo and column chromatography on silica gel (hexanes-EtOAc, 9:1) furnished the desired metathesis product.

General procedure for desilylations with TBAF (reaction $h$ ). The silylated compound (1 mmol) was dissolved in THF $(10 \mathrm{~mL})$ and treated with TBAF $(314 \mathrm{mg}, 1.2 \mathrm{mmol})$. The reaction mixture was then 
stirred at room temp. for $1.5 \mathrm{~h}$. After addition of an aqueous satd. $\mathrm{NH}_{4} \mathrm{Cl}$ solution $(0.1 \mathrm{~mL})$, the mixture was stirred for $5 \mathrm{~min}$. and evaporated in vacuo. The oily residue was then chromatographed on silica gel (hexanes-EtOAc mixtures) to yield the desired product.

General procedure for acid-catalyzed desilylation (reaction $i)$. The silylated compound $(0.3 \mathrm{mmol})$ was dissolved in $\mathrm{MeOH}(15 \mathrm{~mL})$ and treated with PPTS $(15 \mathrm{mg}, 0.06 \mathrm{mmol})$ and water $(0.15 \mathrm{~mL})$. The mixture was then heated at reflux for $18 \mathrm{~h}$, cooled and neutralized by addition of solid $\mathrm{NaHCO}_{3}$. After filtering, the solution was evaporated in vacuo, and the oily residue was subjected to column chromatography on silica gel (EtOAc-MeOH, 19:1). This provided the desired hydroxy compound.

General procedure for hydroboration/oxidation (reaction $j$ ). A solution of the appropriate olefin (10 mmol) in dry THF $(100 \mathrm{~mL})$ was treated under $\mathrm{N}_{2}$ with 9 -BBN $(30 \mathrm{~mL}$ of a $0.5 \mathrm{M}$ THF solution, 15 $\mathrm{mmol})$. The reaction mixture was stirred for $18 \mathrm{~h}$ at room temp. and then quenched by addition of $\mathrm{MeOH}(20 \mathrm{~mL}), 6 \mathrm{M}$ aq $\mathrm{NaOH}(7 \mathrm{~mL})$ and $30 \% \mathrm{H}_{2} \mathrm{O}_{2}(12 \mathrm{~mL})$. The resulting mixture was then stirred at $50^{\circ} \mathrm{C}$ for $1 \mathrm{~h}$ and worked up (extraction with EtOAc). Column chromatography on silica gel (hexanes-EtOAc, 19:1) afforded the desired primary alcohol.

General procedure for the Swern oxidation (reaction $k$ ). DMSO (1.4 $\mathrm{mL}, 20 \mathrm{mmol}$ ) was dissolved under $\mathrm{N}_{2}$ in dry $\mathrm{CH}_{2} \mathrm{Cl}_{2}(20 \mathrm{~mL})$, cooled to $-78^{\circ} \mathrm{C}$ and treated with oxalyl chloride $(875 \mu \mathrm{L}, 10 \mathrm{mmol})$. After stirring at this temp. for $5 \mathrm{~min}$, a solution of the appropriate alcohol $(8 \mathrm{mmol})$ in dry $\mathrm{CH}_{2} \mathrm{Cl}_{2}(6$ $\mathrm{mL}$ ) was added dropwise, followed by triethyl amine $(5.6 \mathrm{~mL}, 40 \mathrm{mmol})$. The reaction mixture was stirred for $15 \mathrm{~min}$. at $-78^{\circ} \mathrm{C}$ and then for further $60 \mathrm{~min}$. at $0^{\circ} \mathrm{C}$. Work-up (extraction with $\mathrm{CH}_{2} \mathrm{Cl}_{2}$ ) and evaporation in vacuo provided an oily residue which was directly used in the next step. 


\section{Synthetic schemes}

\section{Scheme A}

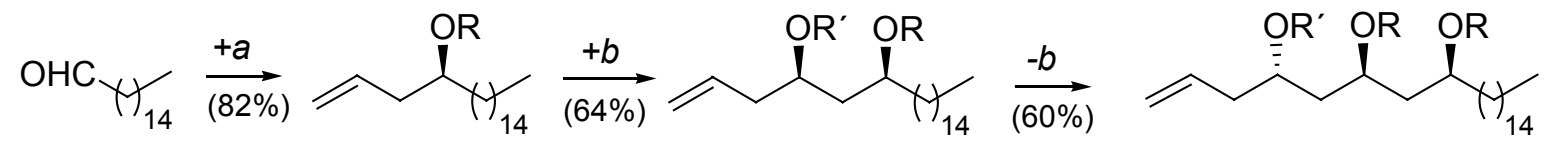

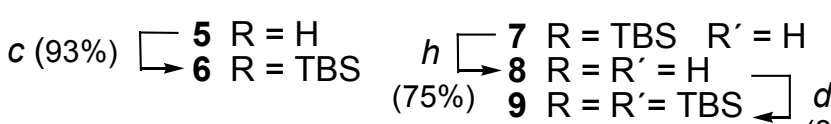
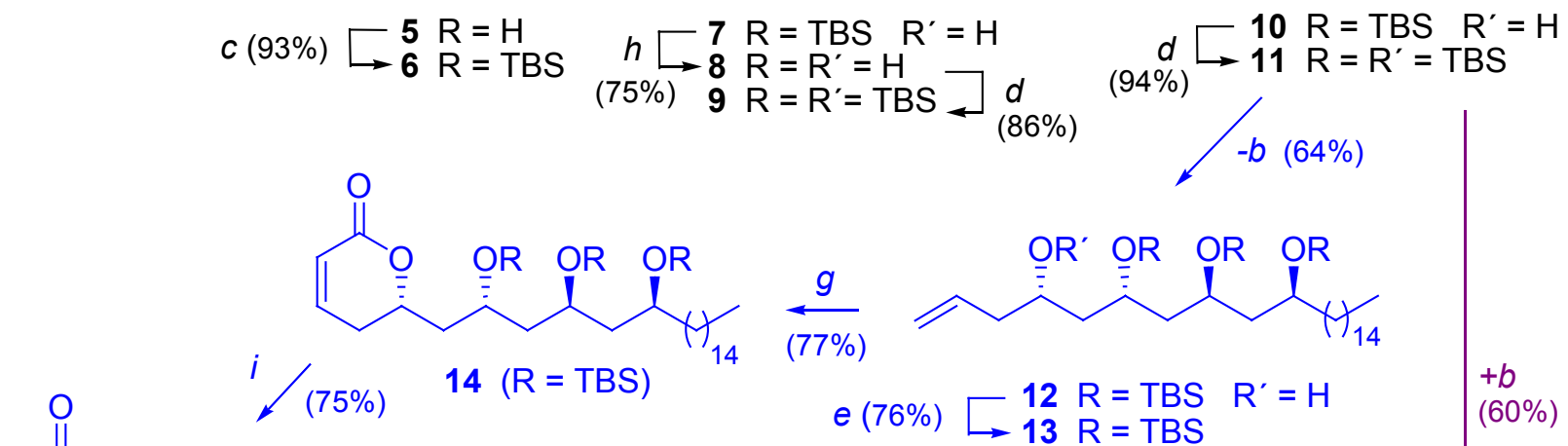<smiles>C=C1C=CC[C@@H](C[C@@H](O)C[C@@H](O)C[C@@H](O)C(C)=[W])O1</smiles>

2 $e(76 \%)$

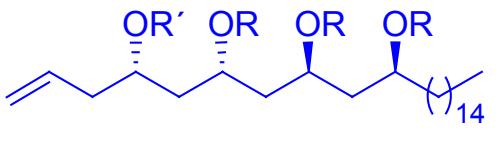

\%) $\square 12 \mathrm{R}=$ TBS $\quad \mathrm{R}^{\prime}=\mathrm{H}$ $\mathrm{R}^{\prime}=(E)-\mathrm{CH}=\mathrm{CHPh}$

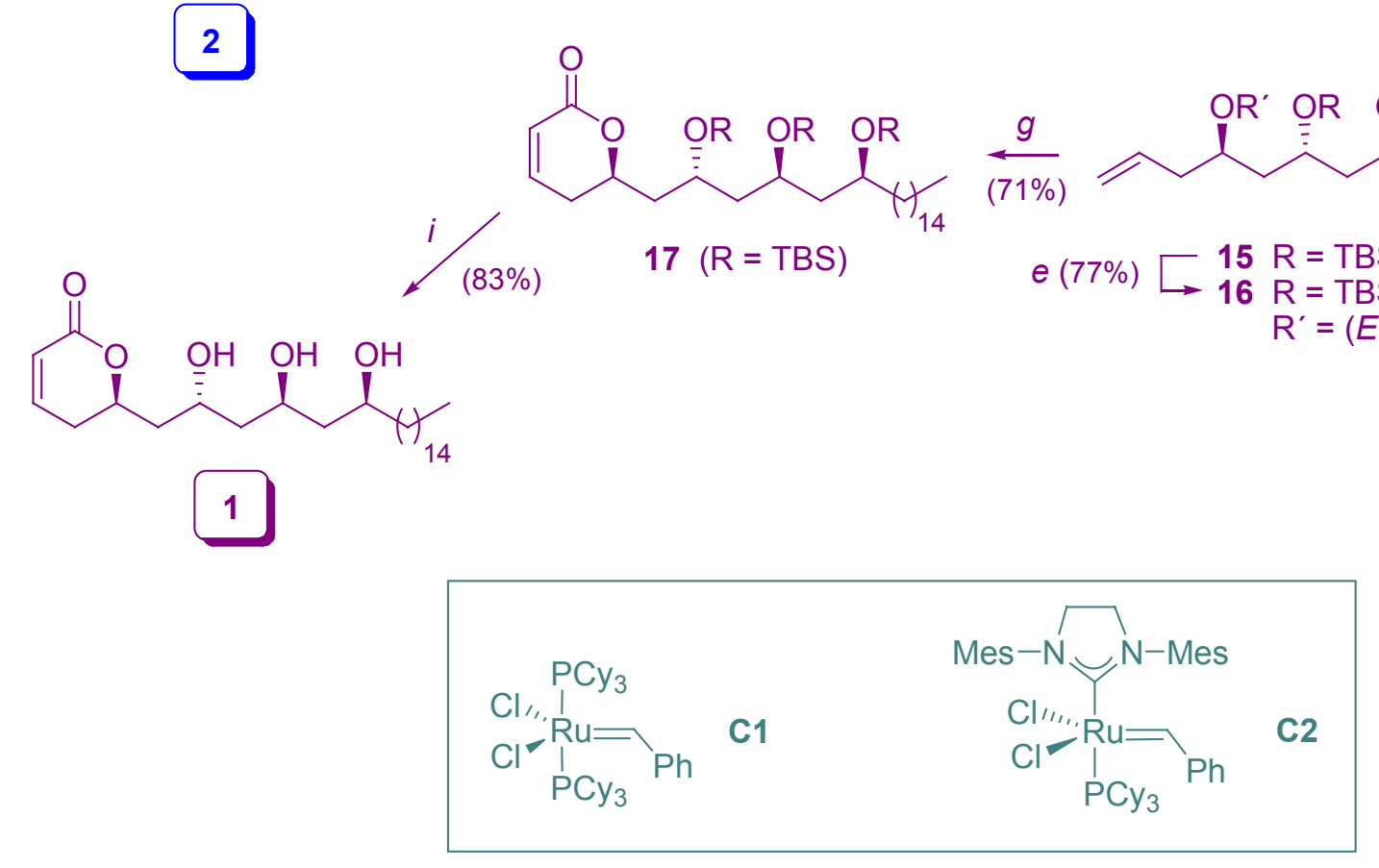




\section{Scheme B}
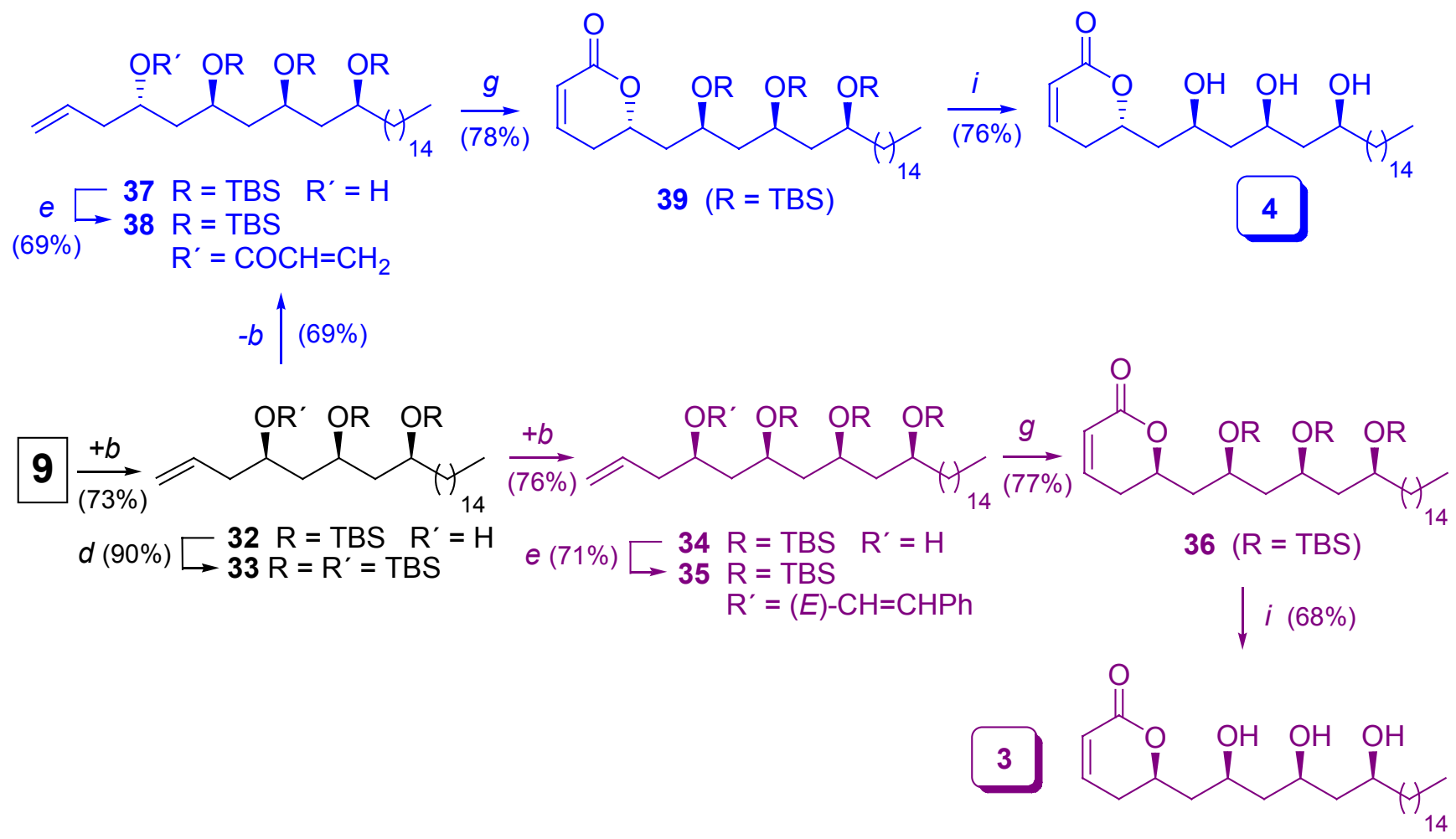


\section{Scheme C}

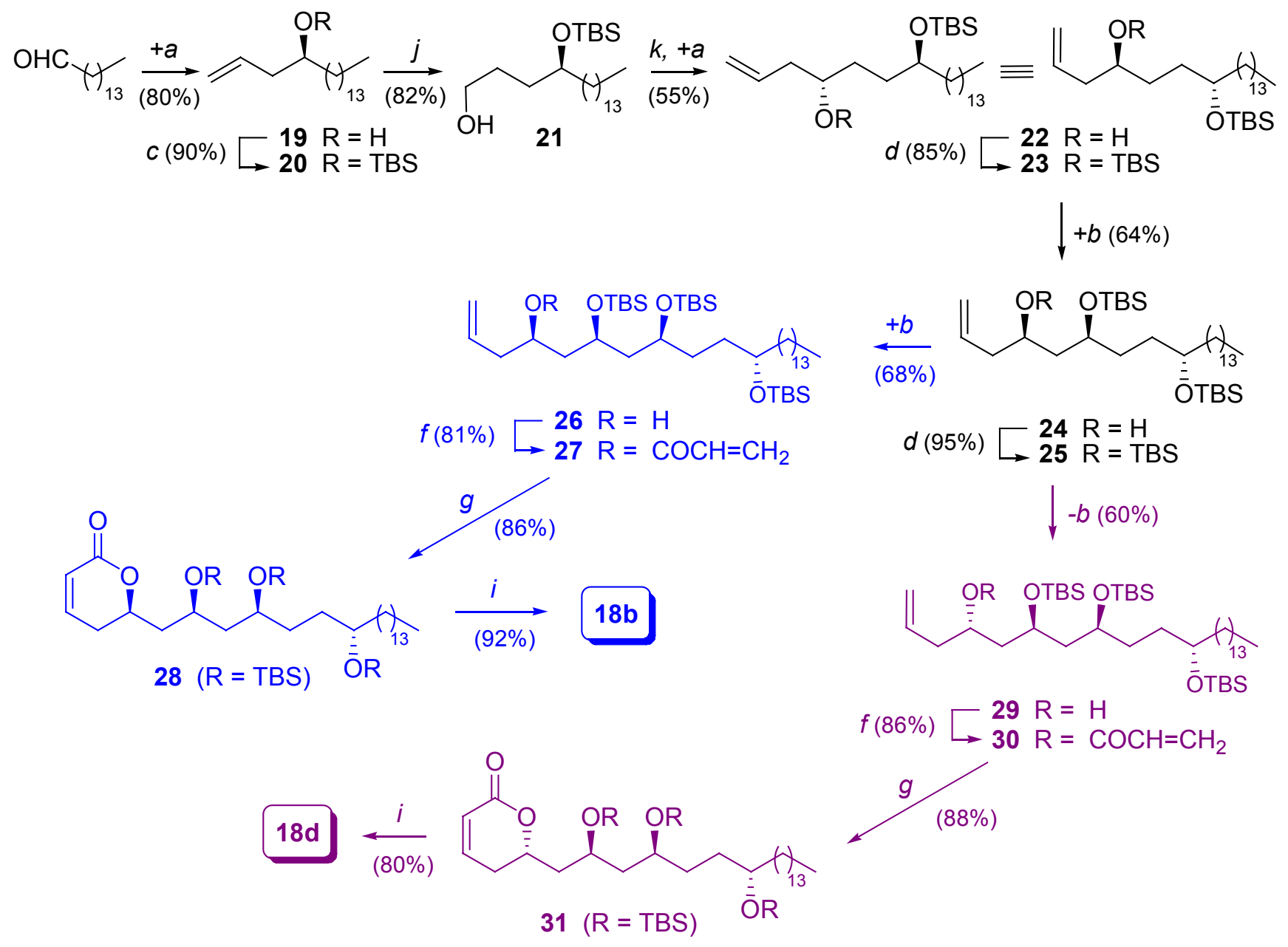




\section{$\underline{\text { Scheme D }}$}

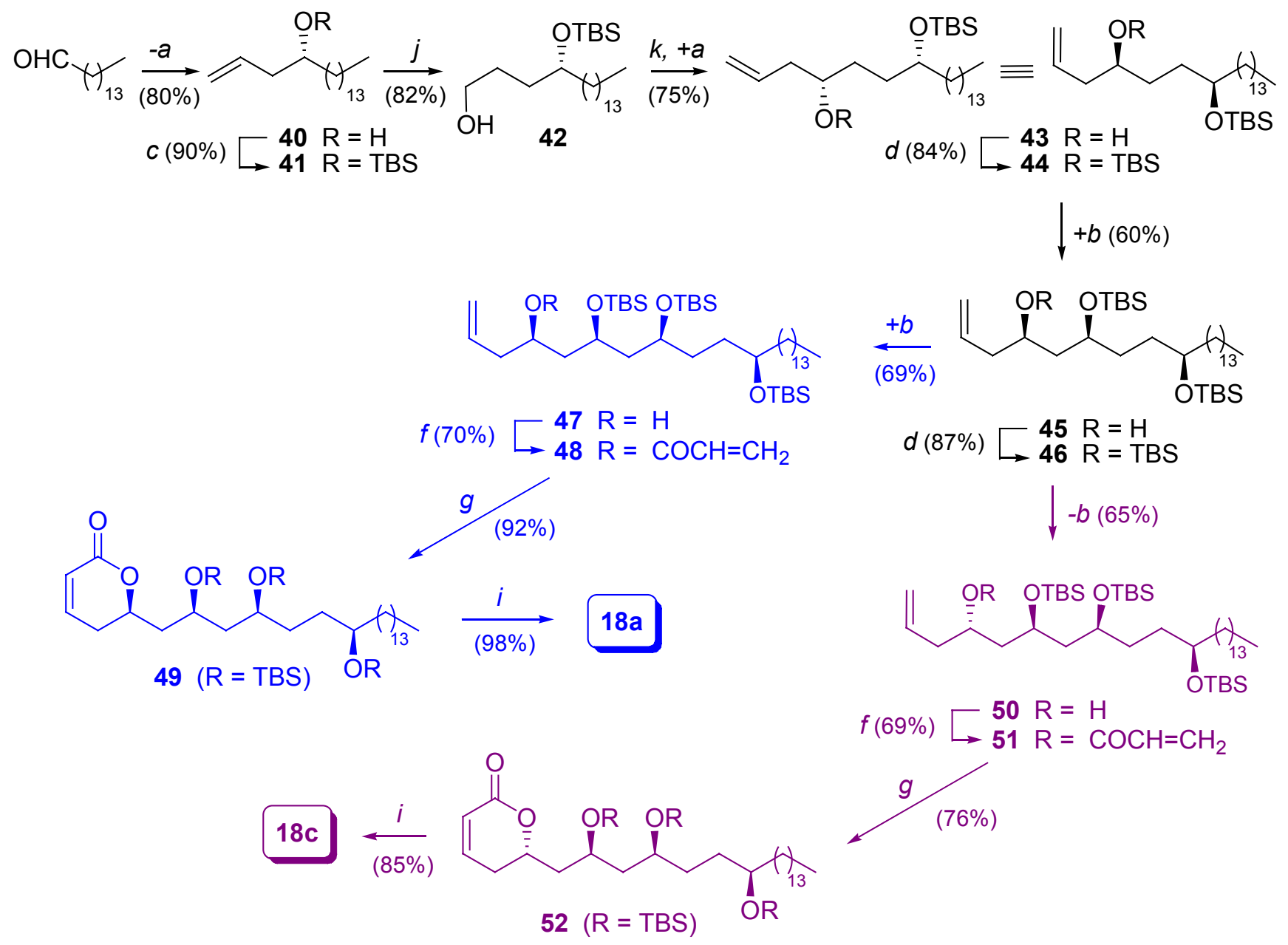




\section{Analytical data of intermediate compounds}

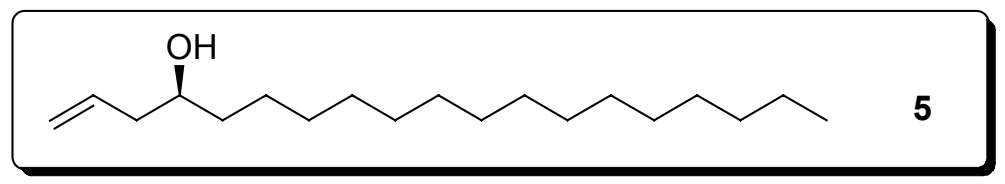

(S)-Nonadec-1-en-4-ol (5): solid, $\mathrm{mp} 42-44^{\circ} \mathrm{C}$ (from hexanes); $[\alpha]_{\mathrm{D}}-2.5$ (c 2.2, $\mathrm{CHCl}_{3}$ ), ${ }^{1} \mathrm{H}$ NMR $(500 \mathrm{MHz}) \delta 5.80(\mathrm{~m}, 1 \mathrm{H}), 5.10(\mathrm{~m}, 2 \mathrm{H}), 3.60(\mathrm{~m}, 1 \mathrm{H}), 2.28(\mathrm{~m}, 1 \mathrm{H}), 2.13(\mathrm{~m}, 1 \mathrm{H}), 2.10(\mathrm{br} \mathrm{s}, 1 \mathrm{H}$, $\mathrm{OH}), 1.50-1.40$ (m, 3H), 1.40-1.20 (br m, 25H), 0.87 (t, $6.5 \mathrm{~Hz}, 3 \mathrm{H}) .{ }^{13} \mathrm{C}$ NMR (125 MHz) $\delta 134.9$, $70.7(\mathrm{CH}), 117.6,41.9,36.8,31.9,29.7$ (several overlapped peaks), 29.3, 25.6, $22.6\left(\mathrm{CH}_{2}\right), 14.0\left(\mathrm{CH}_{3}\right)$. IR $v_{\max } 3330$ (br, OH) cm ${ }^{-1}$. HR EIMS $m / z$ (\% rel. int.) $241.2529\left(\mathrm{M}^{+}-\mathrm{C}_{3} \mathrm{H}_{5}, 36\right), 125$ (26), 111 (54), 97 (92), 83 (100). Calcd. for $\mathrm{C}_{19} \mathrm{H}_{38} \mathrm{O}-\mathrm{C}_{3} \mathrm{H}_{5}, 241.2531$.

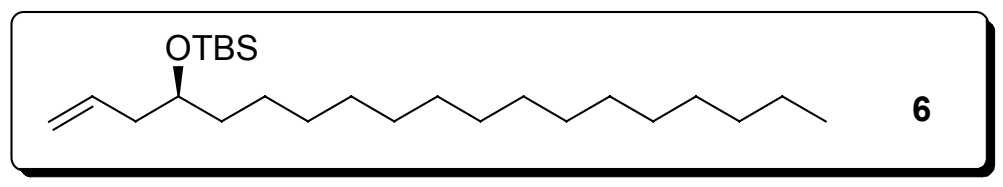

(S)-4-(Tert-butyldimethylsilyloxy)nonadec-1-ene (6): oil; $[\alpha]_{\mathrm{D}}-8.3\left(\right.$ c $\left.2.4, \mathrm{CHCl}_{3}\right) .{ }^{1} \mathrm{H}$ NMR (500 MHz) $\delta 5.80(\mathrm{~m}, 1 \mathrm{H}), 5.05-5.00(\mathrm{~m}, 2 \mathrm{H}), 3.65$ (quint, $6 \mathrm{~Hz}, 1 \mathrm{H}), 2.20(\mathrm{~m}, 2 \mathrm{H}), 1.40(\mathrm{~m}, 3 \mathrm{H}), 1.30-1.20$ (br m, 25H), 0.88 (s, 9H), 0.87 (t, $6.5 \mathrm{~Hz}, 3 \mathrm{H}) ; 0.03$ (s, 6H). ${ }^{13} \mathrm{C}$ NMR $(125 \mathrm{MHz}) \delta 18.2(\mathrm{C}), 135.6$, $72.1(\mathrm{CH}), 116.6,42.0,36.9,32.0,29.8,29.7$ (several overlapped peaks), 29.6, 29.4, 25.4, $22.7\left(\mathrm{CH}_{2}\right)$, 26.0 (x 3), 14.1, -4.3, -4.5 ( $\left.\mathrm{CH}_{3}\right)$. HR EIMS $m / z$ (\% rel. int.) $381.3550\left(\mathrm{M}^{+}-\mathrm{Me}, 3\right), 355(63), 339$ (100). Calcd. for $\mathrm{C}_{25} \mathrm{H}_{52} \mathrm{OSi}-\mathrm{Me}, 381.3552$.

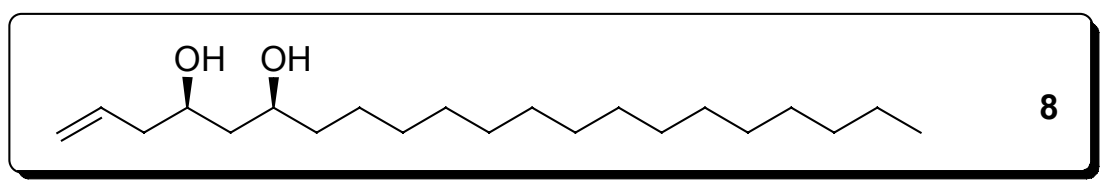

$(4 R, 6 S)$-Henicos-1-ene-4,6-diol (8): obtained in pure form after desilylation of 7 (Scheme A) as a solid, mp 50-52 ${ }^{\circ} \mathrm{C}$ (from hexanes); $[\alpha]_{\mathrm{D}}-2.1\left(c 1.5, \mathrm{CHCl}_{3}\right) .{ }^{1} \mathrm{H}$ NMR $(500 \mathrm{MHz}) \delta 5.80(\mathrm{~m}, 1 \mathrm{H}), 5.10$ (m, 2H), $3.88(\mathrm{~m}, 1 \mathrm{H}), 3.80(\mathrm{~m}, 1 \mathrm{H}), 3.00(\mathrm{br} \mathrm{s}, 2 \mathrm{H}, \mathrm{OH}), 2.20(\mathrm{~m}, 2 \mathrm{H}), 1.50-1.40(\mathrm{~m}, 3 \mathrm{H}), 1.30-1.20$ (br m, 27H), $0.86(\mathrm{t}, 6.5 \mathrm{~Hz}, 3 \mathrm{H}) .{ }^{13} \mathrm{C}$ NMR $(125 \mathrm{MHz}) \delta 134.4,72.9,72.0(\mathrm{CH}), 118.2,42.6,42.5$, 38.2, 32.0, 29.7 (several overlapped peaks), 29.4, 25.4, $22.7\left(\mathrm{CH}_{2}\right), 14.1\left(\mathrm{CH}_{3}\right) . \mathrm{IR} v_{\max } 3330(\mathrm{br}, \mathrm{OH})$ $\mathrm{cm}^{-1}$. HR FABMS $m / z 327.3250\left(\mathrm{M}+\mathrm{H}^{+}\right)$. Calcd. for $\mathrm{C}_{21} \mathrm{H}_{43} \mathrm{O}_{2}, 327.3263$. Anal. Calcd. for $\mathrm{C}_{21} \mathrm{H}_{42} \mathrm{O}_{2}: \mathrm{C}$, 77.24; H, 12.96. Found, C, 77.07; H, 12.83. 


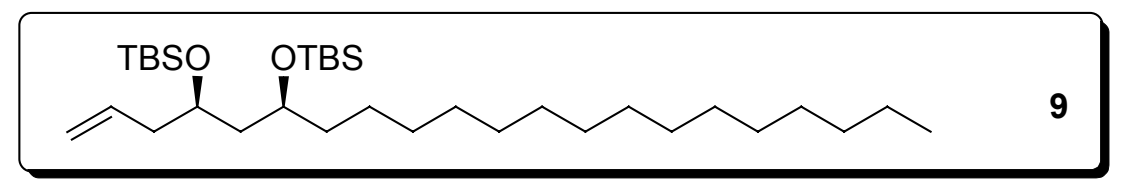

(4R,6S)-4,6-Bis(tert-butyldimethylsilyloxy)henicos-1-ene (9): oil; $[\alpha]_{\mathrm{D}}-6.9$ (c 2.1, $\left.\mathrm{CHCl}_{3}\right) .{ }^{1} \mathrm{H} \mathrm{NMR}$ $(500 \mathrm{MHz}) \delta 5.80(\mathrm{~m}, 1 \mathrm{H}), 5.05-5.00(\mathrm{~m}, 2 \mathrm{H}), 3.80$ (quint, $6 \mathrm{~Hz}, 1 \mathrm{H}), 3.72$ (quint, $6 \mathrm{~Hz}, 1 \mathrm{H}), 2.25(\mathrm{~m}$, $1 \mathrm{H}), 2.15(\mathrm{~m}, 1 \mathrm{H}), 1.60-1.50(\mathrm{~m}, 2 \mathrm{H}), 1.50-1.35(\mathrm{~m}, 3 \mathrm{H}), 1.30-1.20$ (br m, 25H), $0.88(\mathrm{~s}, 18 \mathrm{H}), 0.87(\mathrm{t}$, overlapped, 3H), 0.03 (s, 12H). ${ }^{13} \mathrm{C}$ NMR (125 MHz) $\delta 18.1$ (x 2) (C), 135.2, 69.6, 69.3 (CH), 116.8, 44.6, 41.9, 37.3, 32.0, 29.8, 29.7 (several overlapped peaks), 29.6, 29.4, 25.1, $22.7\left(\mathrm{CH}_{2}\right), 26.0$ (x 6), $14.1,-4.3$ (x 2), -4.4, -4.5 ( $\left.\mathrm{CH}_{3}\right)$. HR EIMS $m / z$ (\% rel. int.) $497.4184\left(\mathrm{M}^{+}-t \mathrm{Bu}, 7\right), 429$ (66), 355 (100), 259 (53). Calcd. for $\mathrm{C}_{33} \mathrm{H}_{70} \mathrm{O}_{2} \mathrm{Si}_{2}-t \mathrm{Bu}, 497.4210$.

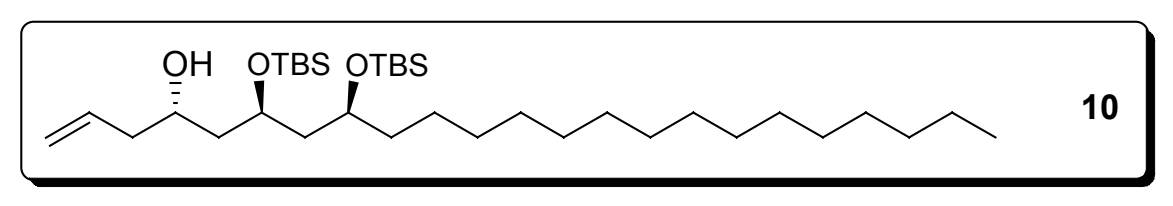

(4S,6R,8S)-6,8-Bis(tert-butyldimethylsilyloxy)tricos-1-en-4-ol (10): oil; $[\alpha]_{\mathrm{D}}+7.3$ (c 2.1, $\left.\mathrm{CHCl}_{3}\right) .{ }^{1} \mathrm{H}$ NMR $(500 \mathrm{MHz}) \delta 5.80(\mathrm{~m}, 1 \mathrm{H}), 5.10-5.05(\mathrm{~m}, 2 \mathrm{H}), 4.16(\mathrm{~m}, 1 \mathrm{H}), 3.96(\mathrm{~m}, 1 \mathrm{H}), 3.59$ (quint, $6 \mathrm{~Hz}$, 1H), 3.50 (br s, 1H, OH), $2.25(\mathrm{~m}, 1 \mathrm{H}), 2.15(\mathrm{~m}, 1 \mathrm{H}), 1.70(\mathrm{~m}, 2 \mathrm{H}), 1.60(\mathrm{~m}, 2 \mathrm{H}), 1.40(\mathrm{~m}, 2 \mathrm{H}), 1.30-$ $1.20($ br m, 26H), $0.88(\mathrm{~s}, 9 \mathrm{H}), 0.87(\mathrm{~s}, 9 \mathrm{H}), 0.87(\mathrm{t}$, overlapped, 3H), $0.08(\mathrm{~s}, 3 \mathrm{H}), 0.07(\mathrm{~s}, 3 \mathrm{H}), 0.03(\mathrm{~s}$, 3H), 0.01 (s, 3H). ${ }^{13} \mathrm{C}$ NMR (125 MHz) $\delta 18.0,17.9$ (C), 135.1, 69.7, 69.3, $67.8(\mathrm{CH}), 117.3,43.0$, 42.4, 40.2, 37.9, 32.0, 29.8, 29.7 (several overlapped peaks), 29.6, 29.4, 25.0, $22.7\left(\mathrm{CH}_{2}\right), 25.9$ (x 3), 25.8 (x 3), 14.1, -4.1, -4.5, -4.6, -4.8 ( $\left(\mathrm{CH}_{3}\right)$. IR $v_{\max } 3510$ (br, OH) cm ${ }^{-1}$. HR EIMS m/z (\% rel. int.) $541.4507\left(\mathrm{M}^{+}-t \mathrm{Bu}, 6\right), 409$ (100), 355 (98), 317 (33), 171 (57), 145 (73). Calcd. for $\mathrm{C}_{35} \mathrm{H}_{74} \mathrm{O}_{3} \mathrm{Si}_{2}-t \mathrm{Bu}_{\text {, }}$ 541.4473.

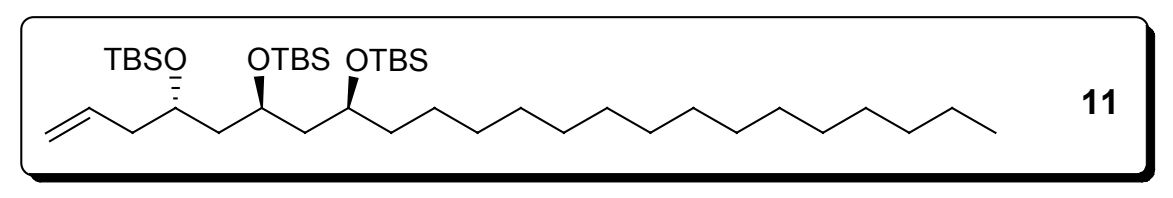

(4S,6S,8S)-4,6,8-Tris(tert-butyldimethylsilyloxy)tricos-1-ene (11): oil; $[\alpha]_{\mathrm{D}}-6.8$ (c 2, $\left.\mathrm{CHCl}_{3}\right) .{ }^{1} \mathrm{H}$ NMR $(500 \mathrm{MHz}) \delta 5.80(\mathrm{~m}, 1 \mathrm{H}), 5.05-5.00(\mathrm{~m}, 2 \mathrm{H}), 3.79(\mathrm{~m}, 3 \mathrm{H}), 2.25(\mathrm{~m}, 1 \mathrm{H}), 2.15(\mathrm{~m}, 1 \mathrm{H}), 1.70-$ $1.40(\mathrm{~m}, 5 \mathrm{H}), 1.40-1.20$ (br m, 27H), $0.88(\mathrm{~s}, 18 \mathrm{H}), 0.87(\mathrm{~s}, 9 \mathrm{H}), 0.87$ (t, overlapped, 3H), $0.05(\mathrm{~s}, 9 \mathrm{H})$, $0.04(\mathrm{~s}, 3 \mathrm{H}), 0.03(\mathrm{~s}, 6 \mathrm{H}) .{ }^{13} \mathrm{C}$ NMR $(125 \mathrm{MHz}) \delta 18.2,18.1,18.0(\mathrm{C}), 135.1,69.6,69.5,67.6(\mathrm{CH})$, 
116.9, 46.1, 45.9, 42.2, 36.7, 32.0, 29.9, 29.7 (several overlapped peaks), 29.4, 25.2, $22.7\left(\mathrm{CH}_{2}\right), 26.0$ (x 9), 14.1, -3.8, -4.1, -4.2 (x 2), -4.3, -4.4 ( $\left.\mathrm{CH}_{3}\right)$. Anal. Calcd. for $\mathrm{C}_{41} \mathrm{H}_{88} \mathrm{O}_{3} \mathrm{Si}_{3}: \mathrm{C}, 69.03 ; \mathrm{H}, 12.43$. Found, C, 69.07; H, 12.66 .

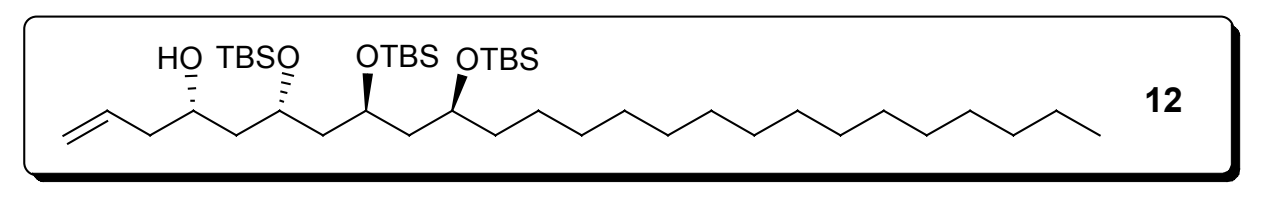

(4S,6S,8S,10S)-6,8,10-Tris(tert-butyldimethylsilyloxy)pentacos-1-en-4-ol (12): oil; $[\alpha]_{\mathrm{D}}-28.4(c$ 2.4, $\left.\mathrm{CHCl}_{3}\right) .{ }^{1} \mathrm{H}$ NMR (500 MHz) $\delta 5.80(\mathrm{~m}, 1 \mathrm{H}), 5.10-5.05(\mathrm{~m}, 2 \mathrm{H}), 3.88(\mathrm{~m}, 1 \mathrm{H}), 3.79(\mathrm{~m}, 2 \mathrm{H}), 3.68$ (m, 1H), 3.10 (br s, 1H, OH), 2.20 (t, $6.5 \mathrm{~Hz}, 2 \mathrm{H}), 1.70-1.40$ (m, 7H), 1.40-1.20 (br m, 27H), 0.88 (s, 18H), 0.87 (s, 9H), 0.87 (t, overlapped, 3H), $0.10(\mathrm{~s}, 6 \mathrm{H}), 0.06(\mathrm{~s}, 3 \mathrm{H}), 0.04(\mathrm{~s}, 6 \mathrm{H}), 0.03(\mathrm{~s}, 3 \mathrm{H}) .{ }^{13} \mathrm{C}$ NMR (125 MHz) $\delta 18.1,18.0,17.9(\mathrm{C}), 134.9,71.0,69.9,69.4,67.4(\mathrm{CH}), 117.5,47.4,45.5,43.1$, 42.1, 36.6, 32.0, 29.9, 29.7 (several overlapped peaks), 29.4, 25.2, $22.7\left(\mathrm{CH}_{2}\right), 26.0$ (x 3), 25.9 (x 3), 25.8 (x 3), 14.1, -3.8, -3.9, -4.2, -4.3, -4.4, $-4.5\left(\mathrm{CH}_{3}\right)$. IR $v_{\max } 3480(\mathrm{br}, \mathrm{OH}) \mathrm{cm}^{-1}$. HR EIMS $\mathrm{m} / z$ (\% rel. int.) $699.5619\left(\mathrm{M}^{+}-t \mathrm{Bu}, 2\right), 567$ (14), 429 (70), 75 (100). Calcd. for $\mathrm{C}_{43} \mathrm{H}_{92} \mathrm{O}_{4} \mathrm{Si}_{3}-t \mathrm{Bu}$, 699.5599 .

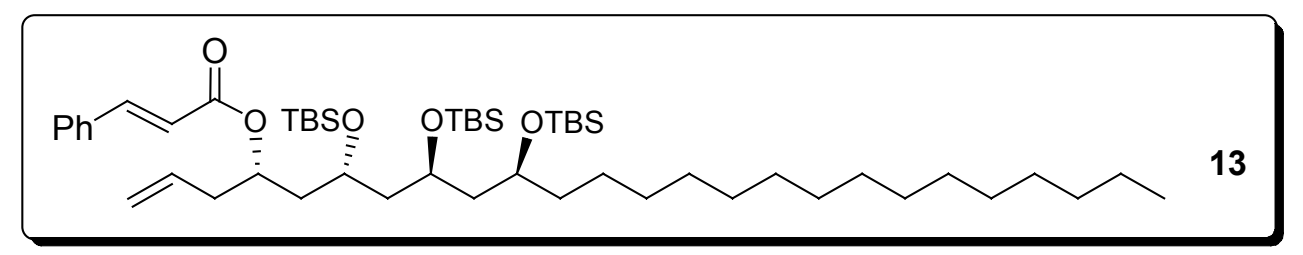

(4S,6S,8S,10S)-6,8,10-Tris(tert-butyldimethylsilyloxy)pentacos-1-en-4-yl (E)-3-phenylacrylate (13): amorphous solid; $[\alpha]_{\mathrm{D}}+1.6\left(\mathrm{c} 2, \mathrm{CHCl}_{3}\right) .{ }^{1} \mathrm{H} \mathrm{NMR}(500 \mathrm{MHz}) \delta 7.66(\mathrm{~d}, 16 \mathrm{~Hz}, 1 \mathrm{H}), 7.50(\mathrm{~m}, 2 \mathrm{H})$, $7.37(\mathrm{~m}, 3 \mathrm{H}), 6.40(\mathrm{~d}, 16 \mathrm{~Hz}, 1 \mathrm{H}), 5.80(\mathrm{~m}, 1 \mathrm{H}), 5.18$ (quint, $6 \mathrm{~Hz}, 1 \mathrm{H}), 5.10-5.05(\mathrm{~m}, 2 \mathrm{H}), 3.90$ (quint, $6 \mathrm{~Hz}, 1 \mathrm{H}), 3.80(\mathrm{~m}, 2 \mathrm{H}), 2.46(\mathrm{~m}, 1 \mathrm{H}), 2.36(\mathrm{~m}, 1 \mathrm{H}), 1.88(\mathrm{~m}, 1 \mathrm{H}), 1.74(\mathrm{~m}, 1 \mathrm{H}), 1.66(\mathrm{~m}, 2 \mathrm{H}), 1.55$ (m, 1H), $1.47(\mathrm{~m}, 1 \mathrm{H}), 1.40-1.20$ (br m, 28H), 0.92 (s, 9H), 0.89 (s, 9H), 0.86 (s, 9H), 0.86 (t, overlapped, 3H), $0.12(\mathrm{~s}, 3 \mathrm{H}), 0.11(\mathrm{~s}, 3 \mathrm{H}), 0.06(\mathrm{~s}, 6 \mathrm{H}), 0.05(\mathrm{~s}, 3 \mathrm{H}), 0.03(\mathrm{~s}, 3 \mathrm{H}) .{ }^{13} \mathrm{C}$ NMR $(125$ MHz) $\delta 166.2,134.6,18.1$ (x 2), 18.0 (C), 144.6, 133.5, 130.2, 128.8 (x 2), 128.1 (x 2), 118.6, 70.9, 69.6, 67.4, 67.1 (CH), 118.0, 46.5, 46.1, 41.8, 38.7, 36.7, 32.0, 29.9, 29.7 (several overlapped peaks), 29.4, 25.2, $22.7\left(\mathrm{CH}_{2}\right), 26.0$ (x 6), 25.9 (x 3), 14.1, -3.8 (x 2), -3.9, -4.1, -4.2, -4.4 $\left(\mathrm{CH}_{3}\right)$. IR $v_{\max }$ $1716(\mathrm{C}=\mathrm{O}), 1639 \mathrm{~cm}^{-1}$. 


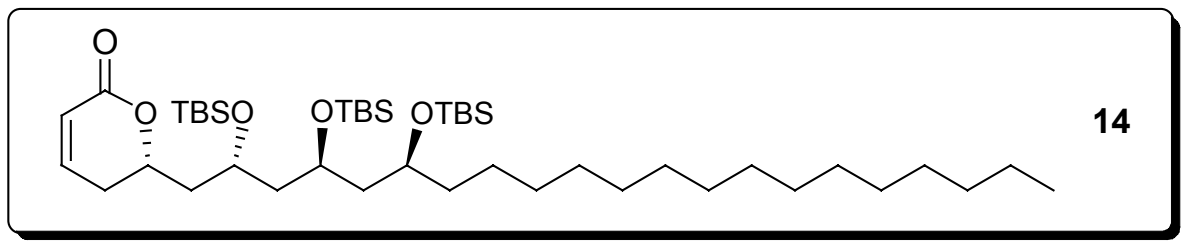

(S)-5,6-Dihydro-6-[(2S,4S,6S)-2,4,6-tris(tert-butyldimethylsilyloxy)henicosyl]pyran-2-one (14): amorphous solid; $[\alpha]_{\mathrm{D}}-37.9\left(c\right.$ 2.4, $\left.\mathrm{CHCl}_{3}\right) .{ }^{1} \mathrm{H} \mathrm{NMR}(500 \mathrm{MHz}) \delta 6.84(\mathrm{~m}, 1 \mathrm{H}), 6.00(\mathrm{br} \mathrm{d}, 9.8 \mathrm{~Hz}$, $1 \mathrm{H}), 4.60(\mathrm{~m}, 1 \mathrm{H}), 3.92$ (quint, $6 \mathrm{~Hz}, 1 \mathrm{H}), 3.78(\mathrm{~m}, 2 \mathrm{H}), 2.40(\mathrm{dt}, 18,4.5 \mathrm{~Hz}, 1 \mathrm{H}), 2.28(\mathrm{~m}, 1 \mathrm{H}), 2.00$ (dt, 14, $7 \mathrm{~Hz}, 1 \mathrm{H}), 1.80-1.40$ (m, 6H), 1.40-1.20 (br m, 27H), 0.88 (s, 9H), 0.87 (s, 9H), 0.86 (s, 9H), $0.86\left(\mathrm{t}\right.$, overlapped, 3H), $0.08(\mathrm{~s}, 3 \mathrm{H}), 0.07(\mathrm{~s}, 3 \mathrm{H}), 0.06(\mathrm{~s}, 3 \mathrm{H}), 0.04(\mathrm{~s}, 6 \mathrm{H}), 0.03(\mathrm{~s}, 3 \mathrm{H}) .{ }^{13} \mathrm{C} \mathrm{NMR}$ $(125 \mathrm{MHz}) \delta 164.2,18.1,18.0$ (x 2) (C), 144.6, 121.7, 75.2, 69.5, 67.5, 66.8 (CH), 46.3, 45.8, 42.4, 36.7, 32.0, 29.9, 29.7 (several overlapped peaks), 29.4, 25.2, $22.7\left(\mathrm{CH}_{2}\right), 25.9$ (x 6), 25.8 (x 3), 14.1, $-3.8,-3.9,-4.0,-4.2,-4.3,-4.4\left(\mathrm{CH}_{3}\right)$. IR $v_{\max } 1740(\mathrm{C}=\mathrm{O}) \mathrm{cm}^{-1}$. HR EIMS $m / z(\%$ rel. int. $)$ $767.5791\left(\mathrm{M}^{+}-\mathrm{Me}, 2\right), 725$ (62), 593 (14), 485 (40), 355 (57), 255 (100).

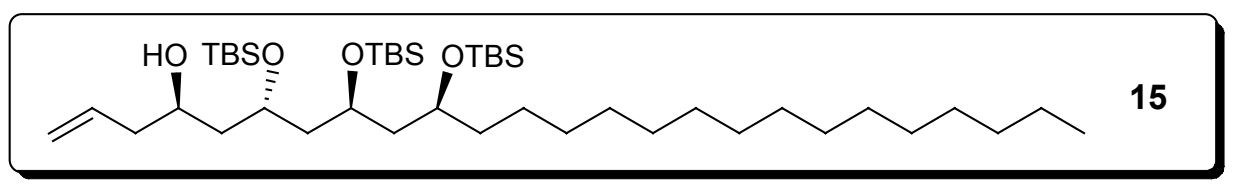

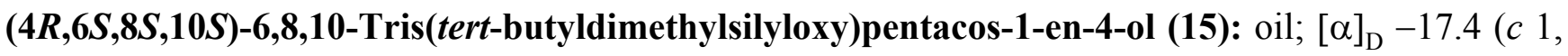
$\left.\mathrm{CHCl}_{3}\right) .{ }^{1} \mathrm{H}$ NMR $(500 \mathrm{MHz}) \delta 5.80(\mathrm{~m}, 1 \mathrm{H}), 5.10-5.05(\mathrm{~m}, 2 \mathrm{H}), 4.10-4.00(\mathrm{~m}, 2 \mathrm{H}), 3.80(\mathrm{~m}, 1 \mathrm{H}), 3.65$ (m, 1H), 3.40 (br s, 1H, OH), $2.25(\mathrm{~m}, 1 \mathrm{H}), 2.16(\mathrm{~m}, 1 \mathrm{H}), 1.85-1.40$ (br m, 7H), 1.40-1.20 (br m, 27H), $0.88(\mathrm{~s}, 27 \mathrm{H}), 0.88(\mathrm{t}$, overlapped, 3H), $0.10(\mathrm{~s}, 3 \mathrm{H}), 0.09(\mathrm{~s}, 3 \mathrm{H}), 0.08(\mathrm{~s}, 3 \mathrm{H}), 0.07(\mathrm{~s}, 3 \mathrm{H}), 0.06(\mathrm{~s}$, $6 \mathrm{H}) .{ }^{13} \mathrm{C}$ NMR $(125 \mathrm{MHz}) \delta 18.1,18.0,17.9$ (C), 134.9, 69.5, 69.4, 67.9, $67.4(\mathrm{CH}), 117.4,45.4,45.0$, 42.4, 41.0, 36.5, 32.0, 29.9, 29.7 (several overlapped peaks), 29.4, 25.2, $22.7\left(\mathrm{CH}_{2}\right), 26.0$ (x 3), 25.9 (x 3), 25.8 (x 3), 14.1, -3.7, -4.2, -4.3, -4.4, -4.5, -4.7 ( $\left.\mathrm{CH}_{3}\right)$. IR $v_{\max } 3500$ (br, OH) cm ${ }^{-1}$. HR EIMS $m / z$ (\% rel. int.) $699.5632\left(\mathrm{M}^{+}-t \mathrm{Bu}, 2\right), 567$ (17), 429 (53), 355 (73), 73 (100). Calcd. for $\mathrm{C}_{43} \mathrm{H}_{92} \mathrm{O}_{4} \mathrm{Si}_{3}-t \mathrm{Bu}, 699.5599$. 


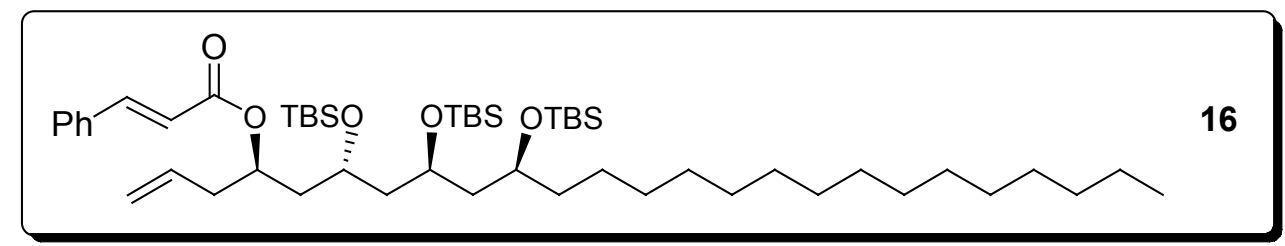

(4R,6S,8S,10S)-6,8,10-Tris(tert-butyldimethylsilyloxy)pentacos-1-en-4-yl (E)-3-phenylacrylate (16): amorphous solid; $[\alpha]_{\mathrm{D}}-24.4\left(c\right.$ 1.2, $\left.\mathrm{CHCl}_{3}\right) .{ }^{1} \mathrm{H}$ NMR $(500 \mathrm{MHz}) \delta 7.67(\mathrm{~d}, 16 \mathrm{~Hz}, 1 \mathrm{H}), 7.54(\mathrm{~m}, 2 \mathrm{H})$, $7.38(\mathrm{~m}, 3 \mathrm{H}), 6.41(\mathrm{~d}, 16 \mathrm{~Hz}, 1 \mathrm{H}), 5.80(\mathrm{~m}, 1 \mathrm{H}), 5.20-5.05(\mathrm{~m}, 3 \mathrm{H}), 3.83(\mathrm{~m}, 2 \mathrm{H}), 3.78$ (quint, $6 \mathrm{~Hz}$, 1H), $2.44(\mathrm{t}, 6 \mathrm{~Hz}, 2 \mathrm{H}), 1.88(\mathrm{~m}, 1 \mathrm{H}), 1.75(\mathrm{~m}, 1 \mathrm{H}), 1.65(\mathrm{~m}, 2 \mathrm{H}), 1.55(\mathrm{~m}, 1 \mathrm{H}), 1.42(\mathrm{~m}, 1 \mathrm{H}), 1.40-$ $1.20($ br m, 28H), $0.92(\mathrm{~s}, 9 \mathrm{H}), 0.90(\mathrm{~s}, 9 \mathrm{H}), 0.89(\mathrm{~s}, 9 \mathrm{H}), 0.88(\mathrm{t}$, overlapped, 3H), $0.12(\mathrm{~s}, 3 \mathrm{H}), 0.10(\mathrm{~s}$, $3 \mathrm{H}), 0.08$ (s, 3H), 0.07 (s, 6H), 0.05 (s, 3H). ${ }^{13} \mathrm{C}$ NMR (125 MHz) $\delta 166.3,134.6,18.1,18.0$ (x 2) (C), $144.5,133.5,130.2,128.8$ (x 2), 128.1 (x 2), 118.6, 70.9, 69.5, 67.6, $67.0(\mathrm{CH}), 117.9,47.2,45.7,41.7$, 39.2, 36.7, 32.0, 29.9, 29.7 (several overlapped peaks), 29.4, 25.1, $22.7\left(\mathrm{CH}_{2}\right), 26.0$ (x 9), 14.1, -3.8, $-3.9,-4.1,-4.2,-4.4,-4.5\left(\mathrm{CH}_{3}\right)$. IR $v_{\max } 1717(\mathrm{C}=\mathrm{O}), 1639 \mathrm{~cm}^{-1}$. HR EIMS m/z (\% rel. int.) $829.6048\left(\mathrm{M}^{+}-t \mathrm{Bu}, 1\right), 267$ (63), 149 (73), 84 (100). Calcd. for $\mathrm{C}_{52} \mathrm{H}_{98} \mathrm{O}_{5} \mathrm{Si}_{3}-t \mathrm{Bu}, 829.6012$.

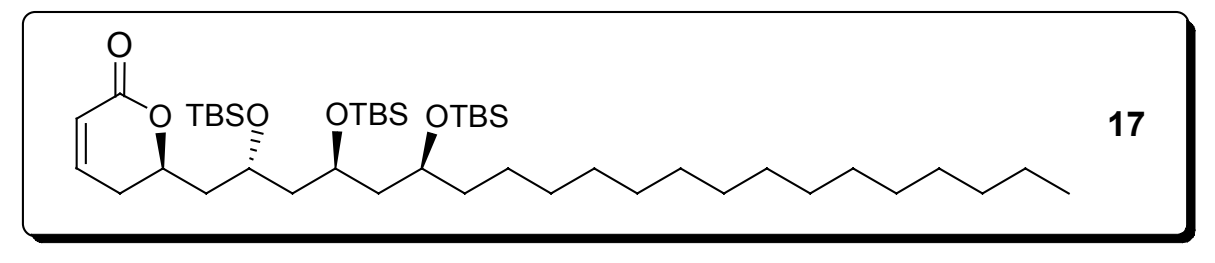

(R)-5,6-Dihydro-6-[(2S,4S,6S)-2,4,6-tris(tert-butyldimethylsilyloxy)henicosyl]pyran-2-one (17): amorphous solid; $[\alpha]_{\mathrm{D}}-11.4\left(c\right.$ 1.8, $\left.\mathrm{CHCl}_{3}\right) .{ }^{1} \mathrm{H} \mathrm{NMR}(500 \mathrm{MHz}) \delta 6.84(\mathrm{~m}, 1 \mathrm{H}), 6.00(\mathrm{br} \mathrm{d}, 9.8 \mathrm{~Hz}$, $1 \mathrm{H}), 4.58(\mathrm{~m}, 1 \mathrm{H}), 4.00(\mathrm{~m}, 1 \mathrm{H}), 3.77(\mathrm{~m}, 2 \mathrm{H}), 2.30(\mathrm{~m}, 2 \mathrm{H}), 2.00(\mathrm{~m}, 1 \mathrm{H}), 1.77(\mathrm{~m}, 1 \mathrm{H}), 1.65-1.55(\mathrm{~m}$, $3 \mathrm{H}), 1.50-1.20$ (br m, 29H), $0.87(\mathrm{~s}, 18 \mathrm{H}), 0.86(\mathrm{~s}, 9 \mathrm{H}), 0.86(\mathrm{t}$, overlapped, 3H), $0.08(\mathrm{~s}, 3 \mathrm{H}), 0.06(\mathrm{~s}$, 3H), 0.05 (s, 3H), 0.04 (s, 6H), 0.02 (s, 3H). ${ }^{13} \mathrm{C}$ NMR (125 MHz) $\delta 163.9,18.1,18.0$ (x 2) (C), 144.8, 121.7, 74.3, 69.5, 67.5, $66.0(\mathrm{CH}), 47.3,45.7,43.0,36.7,32.0,30.0,29.9,29.7$ (several overlapped peaks), 29.6, 25.2, $22.7\left(\mathrm{CH}_{2}\right), 26.0$ (x 3), 25.9 (x 3), 25.8 (x 3), 14.1, -3.9, -4.1, -4.2 (x 2), -4.4, -4.6 $\left(\mathrm{CH}_{3}\right)$. IR $v_{\max } 1740(\mathrm{C}=\mathrm{O}) \mathrm{cm}^{-1}$. HR EIMS m/z (\% rel. int.) $767.5816\left(\mathrm{M}^{+}-\mathrm{Me}, 1\right), 725$ (82), 593 (16), 485 (55), 355 (73), 255 (100). Calcd. for $\mathrm{C}_{44} \mathrm{H}_{90} \mathrm{O}_{5} \mathrm{Si}_{3}-\mathrm{Me}, 767.5855$. 


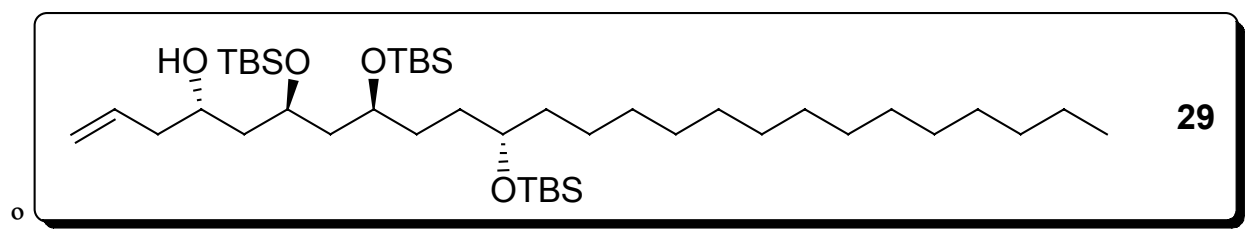

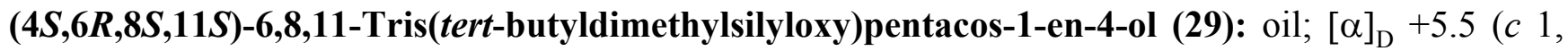
$\left.\mathrm{CHCl}_{3}\right) .{ }^{1} \mathrm{H}$ NMR $(500 \mathrm{MHz}) \delta 5.83(\mathrm{~m}, 1 \mathrm{H}), 5.15-5.05(\mathrm{~m}, 2 \mathrm{H}), 4.17(\mathrm{~m}, 1 \mathrm{H}), 4.00(\mathrm{~m}, 1 \mathrm{H}), 3.62(\mathrm{~m}$, 2H), 3.50 (br s, 1H, OH), 2.27 (dt, 14, 6 Hz, 1H), 2.18 (dt, 14, 6 Hz, 1H), 1.80-1.50 (m, 4H), 1.50-1.40 $(\mathrm{m}, 2 \mathrm{H}), 1.35-1.20$ (br m, 28H), $0.90(\mathrm{~s}, 9 \mathrm{H}), 0.89(\mathrm{~s}, 9 \mathrm{H}), 0.88(\mathrm{~s}, 9 \mathrm{H}), 0.88$ (t, overlapped, $3 \mathrm{H}), 0.11$

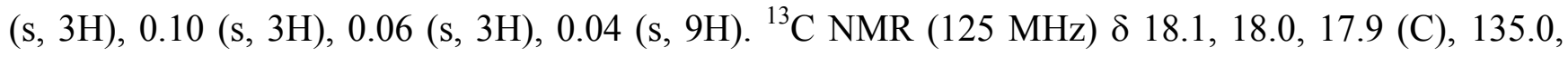
72.4, 69.9, 69.2, $67.8(\mathrm{CH}), 117.3,43.2,42.4$ (x 2), 40.6, 37.0, 33.0, 32.2, 32.0 (x 2), 29.9, 29.7 (several overlapped peaks), 29.4, 25.4, $22.7\left(\mathrm{CH}_{2}\right), 26.0$ (x 3), 25.9 (x 6), 14.1, -4.1, -4.4, -4.5 (x 3), -4.7 $\left(\mathrm{CH}_{3}\right)$. IR $v_{\max } 3500(\mathrm{br}, \mathrm{OH}) \mathrm{cm}^{-1}$. HR EIMS $m / z$ (\% rel. int.) $699.5681\left(\mathrm{M}^{+}-t \mathrm{Bu}, 1\right), 567$ (17), 435 (35), 381 (18), 341 (22), 73 (100). Calcd. for $\mathrm{C}_{43} \mathrm{H}_{92} \mathrm{O}_{4} \mathrm{Si}_{3}-t \mathrm{Bu}, 699.5599$.

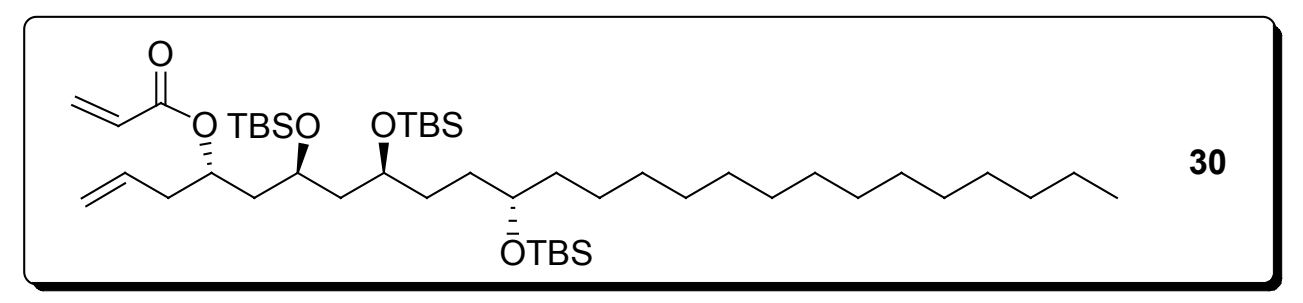

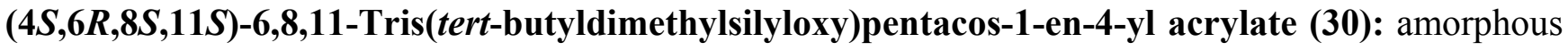
solid; $[\alpha]_{\mathrm{D}}+24\left(c\right.$ 1.2, $\left.\mathrm{CHCl}_{3}\right) .{ }^{1} \mathrm{H}$ NMR $(500 \mathrm{MHz}) \delta 6.39(\mathrm{dd}, 17.3,1.5 \mathrm{~Hz}, 1 \mathrm{H}), 6.09(\mathrm{dd}, 17.3,10.5$ $\mathrm{Hz}, 1 \mathrm{H}), 5.80-5.70(\mathrm{~m}, 2 \mathrm{H}), 5.15-5.05(\mathrm{~m}, 3 \mathrm{H}), 3.88(\mathrm{~m}, 1 \mathrm{H}), 3.71(\mathrm{~m}, 1 \mathrm{H}), 3.63$ (quint, $5.5 \mathrm{~Hz}, 1 \mathrm{H})$, $2.40(\mathrm{~m}, 2 \mathrm{H}), 1.89$ (ddd, 14, 9.5, 3.3 Hz, 1H), 1.75 (ddd, 14, 7.8, $5 \mathrm{~Hz}, 1 \mathrm{H}), 1.60-1.40$ (m, 5H), 1.351.20 (br m, 27H), $0.90(\mathrm{~s}, 9 \mathrm{H}), 0.88(\mathrm{~s}, 18 \mathrm{H}), 0.88(\mathrm{t}$, overlapped, 3H), $0.06(\mathrm{~s}, 6 \mathrm{H}), 0.05(\mathrm{~s}, 6 \mathrm{H}), 0.04$ $(\mathrm{s}, 3 \mathrm{H}), 0.01$ (s, 3H). ${ }^{13} \mathrm{C}$ NMR (125 MHz) $\delta 165.7,18.1,18.0$ (x 2) (C), 133.4, 128.9, 72.5, 71.0, 69.5, $66.4(\mathrm{CH}), 130.2,117.9,45.9,41.3,39.2,37.1,33.1,32.2,32.0,29.9,29.7$ (several overlapped peaks), 29.4, 25.4, $22.7\left(\mathrm{CH}_{2}\right), 26.0$ (x 9), 14.1, -4.1 (x 2), -4.4 (x 2), -4.5,-4.7 ( $\left.\mathrm{CH}_{3}\right)$. IR $v_{\max } 1728(\mathrm{C}=\mathrm{O})$ $\mathrm{cm}^{-1}$. 


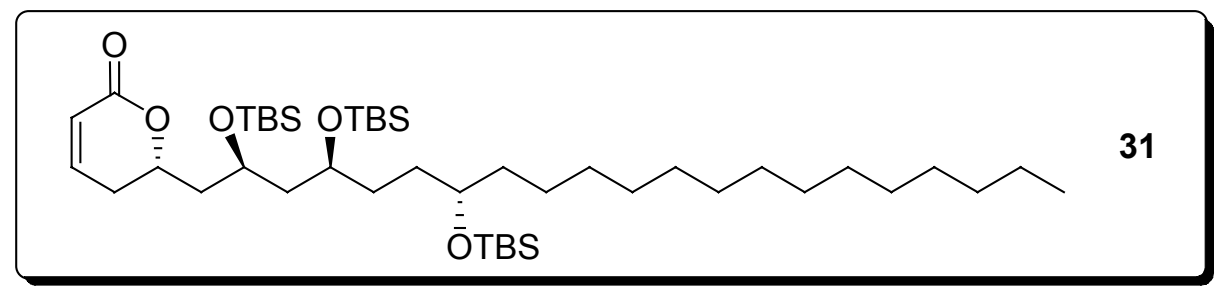

(S)-5,6-Dihydro-6-[(2R,4S,7S)-2,4,7-tris(tert-butyldimethylsilyloxy)henicosyl]pyran-2-one (31): amorphous solid; $[\alpha]_{\mathrm{D}}-2.1\left(c\right.$ 1.3, $\left.\mathrm{CHCl}_{3}\right) .{ }^{1} \mathrm{H}$ NMR $(500 \mathrm{MHz}) \delta 6.85(\mathrm{~m}, 1 \mathrm{H}), 5.99($ br d, $9.7 \mathrm{~Hz}$, 1H), $4.58(\mathrm{~m}, 1 \mathrm{H}), 4.14(\mathrm{~m}, 1 \mathrm{H}), 3.75(\mathrm{~m}, 1 \mathrm{H}), 3.63$ (quint, $5.5 \mathrm{~Hz}, 1 \mathrm{H}), 2.30(\mathrm{~m}, 2 \mathrm{H}), 2.02$ (dd, 14, 9.5, $2.5 \mathrm{~Hz}, 1 \mathrm{H}), 1.74$ (ddd, 14, 7.5, $4.5 \mathrm{~Hz}, 1 \mathrm{H}), 1.61$ (ddd, 14, 9.6, $3 \mathrm{~Hz}, 1 \mathrm{H}), 1.55-1.40(\mathrm{~m}, 3 \mathrm{H})$, 1.35-1.20 (br m, 26H), 0.90 (s, 9H), 0.89 (s, 9H), 0.88 (s, 9H), 0.88 (t, overlapped, 3H), 0.09 (s, 3H), 0.08 (s, 3H), 0.07 (s, 3H), 0.06 (s, 3H), 0.04 (s, 6H). ${ }^{13} \mathrm{C}$ NMR (125 MHz) $\delta 163.9,18.1,18.0$ (x 2) (C), 144.8, 121.7, 74.5, 72.4, 69.4, $65.5(\mathrm{CH}), 45.8,42.9,37.0,33.2,32.3,32.0,30.1,29.8,29.7$ (several overlapped peaks), 29.3, 25.3, $22.7\left(\mathrm{CH}_{2}\right), 25.9$ (x 9), 14.1, -4.2 (x 2), -4.4 (x 2), -4.5, -4.7 ( $\left.\mathrm{CH}_{3}\right) . \mathrm{IR}$ $v_{\max } 1722(\mathrm{C}=\mathrm{O}) \mathrm{cm}^{-1}$.

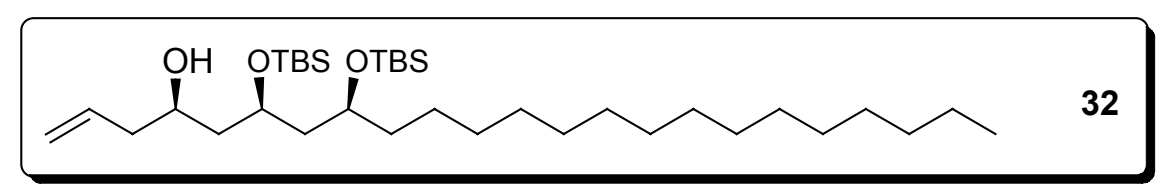

(4R,6R,8S)-6,8-Bis(tert-butyldimethylsilyloxy)tricos-1-en-4-ol (32): oil; $[\alpha]_{\mathrm{D}}+20\left(c \quad 1.4, \mathrm{CHCl}_{3}\right) .{ }^{1} \mathrm{H}$ NMR (500 MHz) $\delta 5.80(\mathrm{~m}, 1 \mathrm{H}), 5.10-5.05(\mathrm{~m}, 2 \mathrm{H}), 4.05(\mathrm{~m}, 1 \mathrm{H}), 3.80(\mathrm{~m}, 1 \mathrm{H}), 3.67(\mathrm{~m}, 1 \mathrm{H}), 3.30$ (br s, 1H, OH), $2.25(\mathrm{~m}, 2 \mathrm{H}), 1.80-1.65(\mathrm{~m}, 2 \mathrm{H}), 1.60-1.40(\mathrm{~m}, 3 \mathrm{H}), 1.35-1.20(\mathrm{br} \mathrm{m}, 27 \mathrm{H}), 0.90(\mathrm{~s}$, 9H), 0.88 (s, 9H), 0.87 (t, overlapped, 3H), $0.11(\mathrm{~s}, 6 \mathrm{H}), 0.08(\mathrm{~s}, 3 \mathrm{H}), 0.07(\mathrm{~s}, 3 \mathrm{H}) .{ }^{13} \mathrm{C}$ NMR $(125$ $\mathrm{MHz}) \delta$ 18.0, $17.8(\mathrm{C}), 134.9,70.6,70.1,69.5(\mathrm{CH}), 117.3,45.6,42.5,42.3,37.9,32.0,29.8,29.7$ (several overlapped peaks), 29.6, 29.4, 24.7, $22.7\left(\mathrm{CH}_{2}\right), 25.9$ (x 3), 25.8 (x 3), 14.1, -4.0, -4.1, -4.5, $-4.7\left(\mathrm{CH}_{3}\right)$. IR $v_{\max } 3350(\mathrm{br}, \mathrm{OH}) \mathrm{cm}^{-1}$. 


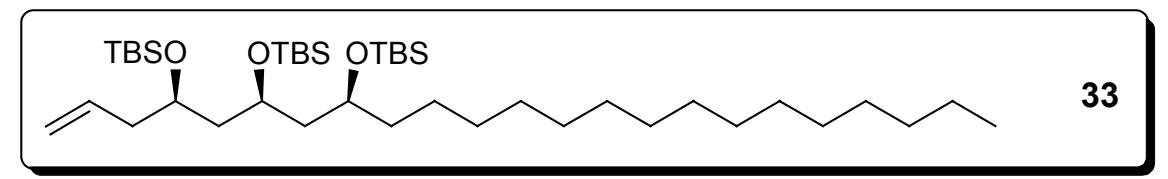

(4R,6S,8S)-4,6,8-Tris(tert-butyldimethylsilyloxy)tricos-1-ene (33): oil; $[\alpha]_{\mathrm{D}}-8.8\left(\mathrm{c} 1.3, \mathrm{CHCl}_{3}\right) .{ }^{1} \mathrm{H}$ NMR (500 MHz) $\delta 5.80(\mathrm{~m}, 1 \mathrm{H}), 5.07-5.00(\mathrm{~m}, 2 \mathrm{H}), 3.85-3.75(\mathrm{~m}, 3 \mathrm{H}), 2.30(\mathrm{~m}, 1 \mathrm{H}), 2.15(\mathrm{~m}, 1 \mathrm{H})$, 1.70-1.45 (m, 4H), 1.40-1.20 (br m, 28H), 0.90 (s, 27H), 0.87 (t, overlapped, 3H), $0.07(\mathrm{~s}, 18 \mathrm{H}) .{ }^{13} \mathrm{C}$ NMR (125 MHz) $\delta 18.2,18.1,18.0(\mathrm{C}), 135.2,69.7,69.3,67.3(\mathrm{CH}), 116.9,46.0,45.8,42.1,37.4$, 32.0, 30.0, 29.7 (several overlapped peaks), 29.5, 25.2, $22.7\left(\mathrm{CH}_{2}\right), 26.0$ (x 9), 14.1, -4.1, -4.2, -4.3 (x 3), -4.4 $\left(\mathrm{CH}_{3}\right)$. HR EIMS m/z (\% rel. int.) $697.5816\left(\mathrm{M}^{+}-\mathrm{Me}, 1\right), 655$ (6), 523 (10), 429 (35), 355 (100), 185 (66). Calcd. for $\mathrm{C}_{41} \mathrm{H}_{88} \mathrm{O}_{3} \mathrm{Si}_{3}-\mathrm{Me}$, 697.5806. Anal. Calcd. for $\mathrm{C}_{41} \mathrm{H}_{88} \mathrm{O}_{3} \mathrm{Si}_{3}$ : C, 69.03; $\mathrm{H}$, 12.43. Found, C, 68.91; H, 12.29.

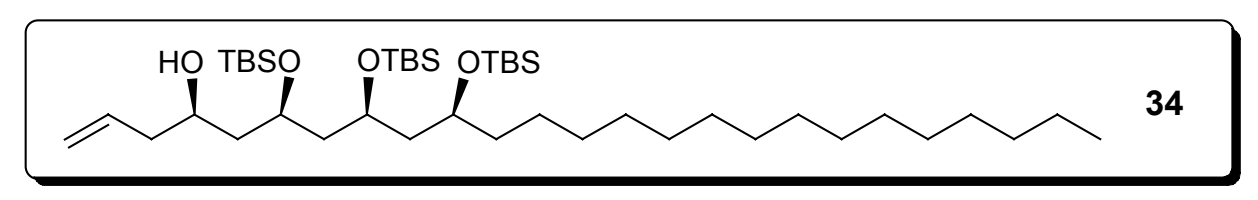

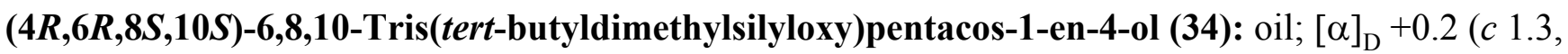
$\left.\mathrm{CHCl}_{3}\right) .{ }^{1} \mathrm{H}$ NMR $(500 \mathrm{MHz}) \delta 5.80(\mathrm{~m}, 1 \mathrm{H}), 5.10-5.05(\mathrm{~m}, 2 \mathrm{H}), 4.10(\mathrm{~m}, 1 \mathrm{H}), 3.80(\mathrm{~m}, 2 \mathrm{H}), 3.64(\mathrm{~m}$, 1H), 3.20 (br s, 1H, OH), 2.25 (m, 1H), 2.17 (m, 1H), 1.80 (br d, 14 Hz, 1H), 1.70-1.60 (m, 2H), 1.501.35 (br m, 3H), 1.35-1.20 (br m, 28H), 0.89 (s, 18H), 0.88 (s, 9H), 0.88 (t, overlapped, 3H), $0.11(\mathrm{~s}$, $6 \mathrm{H}), 0.04(\mathrm{~s}, 9 \mathrm{H}), 0.00$ (s, 3H). ${ }^{13} \mathrm{C} \mathrm{NMR}(125 \mathrm{MHz}) \delta 18.1,18.0,17.9(\mathrm{C}), 134.9,71.2,70.7,69.6$, $67.1(\mathrm{CH}), 117.5,46.4,46.2,42.3$ (x 2), 37.9, 32.0, 29.9, 29.7 (several overlapped peaks), 29.4, 24.9, $22.7\left(\mathrm{CH}_{2}\right), 26.0$ (x 3), 25.9 (x 6), 14.1, -3.8, -3.9, -4.0, -4.2, -4.5, -4.6 ( $\left.\mathrm{CH}_{3}\right)$. IR $v_{\max } 3350(\mathrm{br}, \mathrm{OH})$ $\mathrm{cm}^{-1}$. HR EIMS $m / z$ (\% rel. int.) $699.5534\left(\mathrm{M}^{+}-t \mathrm{Bu}, 2\right), 567$ (7), 429 (18), 355 (99), 185 (100), 73 (95). Calcd. for $\mathrm{C}_{43} \mathrm{H}_{92} \mathrm{O}_{4} \mathrm{Si}_{3}-t \mathrm{Bu}, 699.5599$.

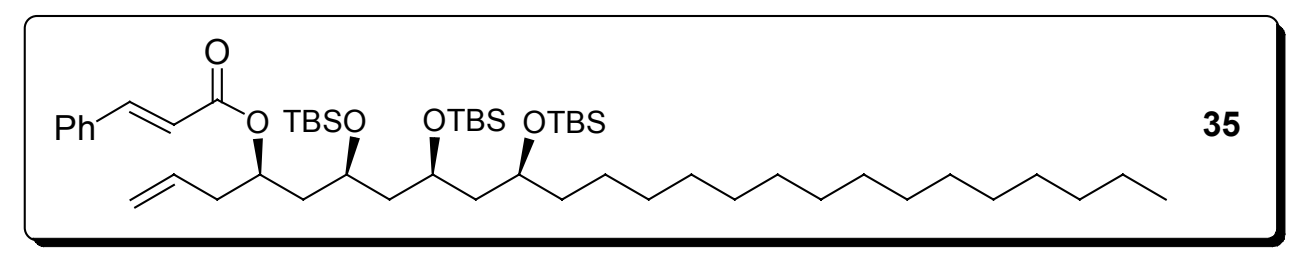

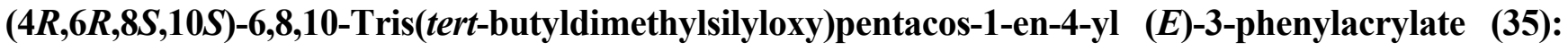
amorphous solid; $[\alpha]_{\mathrm{D}}-22.8\left(c\right.$ 1.3, $\left.\mathrm{CHCl}_{3}\right) .{ }^{1} \mathrm{H} \mathrm{NMR}(500 \mathrm{MHz}) \delta 7.67(\mathrm{~d}, 16 \mathrm{~Hz}, 1 \mathrm{H}), 7.50(\mathrm{~m}, 2 \mathrm{H})$, 
$7.37(\mathrm{~m}, 3 \mathrm{H}), 6.39(\mathrm{~d}, 16 \mathrm{~Hz}, 1 \mathrm{H}), 5.80(\mathrm{~m}, 1 \mathrm{H}), 5.18(\mathrm{~m}, 1 \mathrm{H}), 5.10-5.05(\mathrm{~m}, 2 \mathrm{H}), 3.80(\mathrm{~m}, 3 \mathrm{H}), 2.43$ (m, 1H), $2.33(\mathrm{~m}, 1 \mathrm{H}), 1.85$ (ddd, 14, 8, $5.3 \mathrm{~Hz}, 1 \mathrm{H}), 1.77$ (ddd, 14, 6.5, $5 \mathrm{~Hz}, 1 \mathrm{H}), 1.65(\mathrm{t}, 6 \mathrm{~Hz}, 1 \mathrm{H})$, 1.57 (t, $6 \mathrm{~Hz}, 1 \mathrm{H}), 1.55-1.40$ (m, 2H), 1.40-1.20 (br m, 28H), 0.92 (s, 9H), 0.90 (s, 9H), 0.89 (s, 9H), 0.88 (t, overlapped, 3H), $0.10(\mathrm{~s}, 3 \mathrm{H}), 0.08(\mathrm{~s}, 3 \mathrm{H}), 0.07(\mathrm{~s}, 3 \mathrm{H}), 0.05(\mathrm{~s}, 3 \mathrm{H}), 0.04(\mathrm{~s}, 6 \mathrm{H}) .{ }^{13} \mathrm{C}$ NMR (125 MHz) $\delta 166.1,134.6,18.1,18.0,17.9$ (C), 144.7, 133.6, 130.2, 128.8 (x 2), 128.1 (x 2), 118.5, 70.7, 69.7, 67.1, $66.9(\mathrm{CH}), 117.9,46.1,45.6,41.9,39.3,37.0,32.0,29.9,29.7$ (several overlapped peaks), 29.4, 25.4, $22.7\left(\mathrm{CH}_{2}\right), 26.0$ (x 9), 14.1, -4.1 (x 2), -4.2 (x 2), -4.3 (x 2) $\left(\mathrm{CH}_{3}\right)$. IR $v_{\max } 1715$ $(\mathrm{C}=\mathrm{O}), 1637 \mathrm{~cm}^{-1}$. HR FABMS $m / z 887.6810\left(\mathrm{M}+\mathrm{H}^{+}\right)$. Calcd. for $\mathrm{C}_{52} \mathrm{H}_{99} \mathrm{O}_{5} \mathrm{Si}_{3}, 887.6795$.

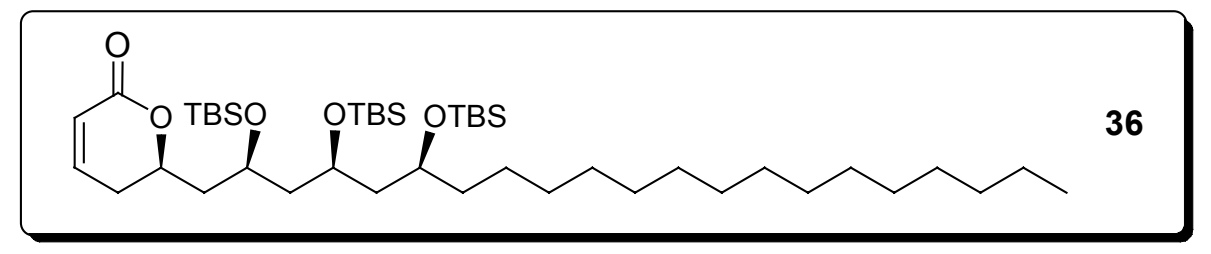

(R)-5,6-Dihydro-6-[(2R,4S,6S)-2,4,6-tris(tert-butyldimethylsilyloxy)henicosyl]pyran-2-one (36): amorphous solid; $[\alpha]_{\mathrm{D}}+15.5\left(c 0.9, \mathrm{CHCl}_{3}\right) .{ }^{1} \mathrm{H}$ NMR $(500 \mathrm{MHz}) \delta 6.85(\mathrm{~m}, 1 \mathrm{H}), 6.00(\mathrm{dd}, 9.8,2 \mathrm{~Hz}$, 1H), $4.63(\mathrm{~m}, 1 \mathrm{H}$ ), 4.00 (quint, $6 \mathrm{~Hz}, 1 \mathrm{H}$ ), 3.83 (quint, $6 \mathrm{~Hz}, 1 \mathrm{H}$ ), 3.75 (quint, $6 \mathrm{~Hz}, 1 \mathrm{H}$ ), 2.42 (ddd, 18, 5.5, $4.5 \mathrm{~Hz} 1 \mathrm{H}$ ), 2.30 (ddt, 18, 11, $1.5 \mathrm{~Hz}, 1 \mathrm{H}), 2.00$ (dt, 14, $7 \mathrm{~Hz}, 1 \mathrm{H}), 1.88$ (ddd, 14, 7, $5 \mathrm{~Hz}, 1 \mathrm{H}$ ), 1.75-1.45 (br m, 5H), 1.40-1.20 (br m, 29H), 0.89 (s, 9H), 0.88 (s, 18H), 0.87 (t, overlapped, 3H), 0.09 (s, 3H), 0.07 (s, 3H), 0.06 (s, 9H), 0.05 (s, 3H). ${ }^{13} \mathrm{C}$ NMR (125 MHz) $\delta 164.1,18.1,18.0$ (x 2) (C), 144.6, 121.7, 75.3, 69.5, 67.0, 66.2 (CH), 45.8, 45.7, 41.9, 37.5, 32.0, 29.8, 29.7 (several overlapped peaks), 29.4, 25.2, $22.7\left(\mathrm{CH}_{2}\right), 25.9$ (x 6), 25.8 (x 3), 14.1, -4.2 (x 3), -4.3 (x 2), -4.4 $\left(\mathrm{CH}_{3}\right)$. IR $v_{\max }$ $1738(\mathrm{C}=\mathrm{O}) \mathrm{cm}^{-1}$.

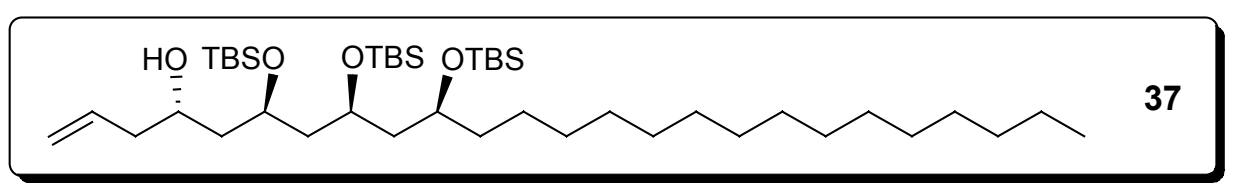

(4S,6R,8S,10S)-6,8,10-Tris(tert-butyldimethylsilyloxy)pentacos-1-en-4-ol (37): oil; $[\alpha]_{\mathrm{D}}+7.9(c$ 1.8, $\left.\mathrm{CHCl}_{3}\right) .{ }^{1} \mathrm{H}$ NMR $(500 \mathrm{MHz}) \delta 5.80(\mathrm{~m}, 1 \mathrm{H}), 5.10-5.05(\mathrm{~m}, 2 \mathrm{H}), 4.18(\mathrm{~m}, 1 \mathrm{H}), 4.00(\mathrm{~m}, 1 \mathrm{H}), 3.72(\mathrm{~m}$, $2 \mathrm{H}), 2.20(\mathrm{~m}, 2 \mathrm{H}), 1.83(\mathrm{~m}, 1 \mathrm{H}), 1.70-1.60(\mathrm{~m}, 2 \mathrm{H}), 1.60-1.50(\mathrm{~m}, 2 \mathrm{H}), 1.45-1.20(\mathrm{br} \mathrm{m}, 28 \mathrm{H}), 0.88(\mathrm{~s}$, $18 \mathrm{H}), 0.87$ (s, 9H), 0.87 (t, overlapped, 3H), 0.10 (s, 3H), 0.09 (s, 3H), 0.03 (s, 12H). ${ }^{13} \mathrm{C}$ NMR $(125$ $\mathrm{MHz}) \delta 18.1,18.0$ (x 2) (C), 135.0, 69.7, 68.9, 67.7, $67.2(\mathrm{CH}), 117.3,46.0,44.4,42.5,40.9,37.6$, 32.0, 29.9, 29.7 (several overlapped peaks), 29.4, 25.1, $22.7\left(\mathrm{CH}_{2}\right), 26.0$ (x 3), 25.9 (x 6), 14.1, -4.1, -4.2 (x 2), $-4.3,-4.4,-4.7\left(\mathrm{CH}_{3}\right)$. IR $v_{\max } 3350(\mathrm{br}, \mathrm{OH}) \mathrm{cm}^{-1}$. 


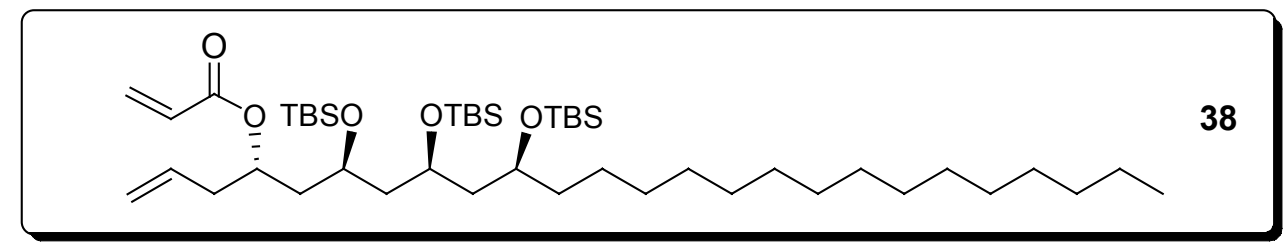

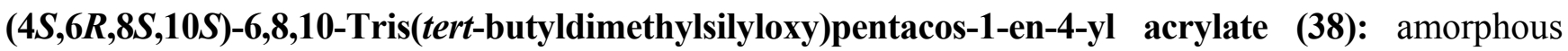
solid; $[\alpha]_{\mathrm{D}}+21.9\left(c\right.$ 0.3, $\left.\mathrm{CHCl}_{3}\right) .{ }^{1} \mathrm{H}$ NMR $(500 \mathrm{MHz}) \delta 6.39$ (br d, $\left.17.3 \mathrm{~Hz}, 1 \mathrm{H}\right), 6.09$ (dd, 17.3, 10.4 $\mathrm{Hz}, 1 \mathrm{H}), 5.80-5.70(\mathrm{~m}, 2 \mathrm{H}), 5.10-5.00(\mathrm{~m}, 3 \mathrm{H}), 3.90(\mathrm{~m}, 1 \mathrm{H}), 3.79(\mathrm{~m}, 1 \mathrm{H}), 3.68(\mathrm{~m}, 1 \mathrm{H}), 2.39(\mathrm{~m}$, 2H), $1.92(\mathrm{~m}, 1 \mathrm{H}), 1.70-1.40(\mathrm{~m}, 5 \mathrm{H}), 1.40-1.20$ (br m, 28H), 0.89 (s, 27H), 0.88 (t, overlapped, 3H), $0.08(\mathrm{~s}, 6 \mathrm{H}), 0.07(\mathrm{~s}, 6 \mathrm{H}), 0.03(\mathrm{~s}, 3 \mathrm{H}), 0.00(\mathrm{~s}, 3 \mathrm{H}) .{ }^{13} \mathrm{C} \mathrm{NMR}(125 \mathrm{MHz}) \delta 165.8,18.1,18.0,17.9(\mathrm{C})$, 133.3, 129.0, 71.0, 69.7, 67.0, 66.1 (CH), 130.3, 117.9, 46.5, 46.0, 41.2, 39.2, 37.7, 32.0, 29.9, 29.7 (several overlapped peaks), 29.4, 25.0, $22.7\left(\mathrm{CH}_{2}\right), 26.0$ (x 9), 14.1, -4.1 (x 3), -4.3 (x 2), -4.7 $\left(\mathrm{CH}_{3}\right)$. IR $v_{\max } 1723(\mathrm{C}=\mathrm{O}), 1639 \mathrm{~cm}^{-1}$. HR EIMS $m / z$ (\% rel. int.) $753.5757\left(\mathrm{M}^{+}-t \mathrm{Bu}, 1\right), 355$ (100), 211 (47). Calcd. for $\mathrm{C}_{46} \mathrm{H}_{94} \mathrm{O}_{5} \mathrm{Si}_{3}-t \mathrm{Bu}, 753.5705$.

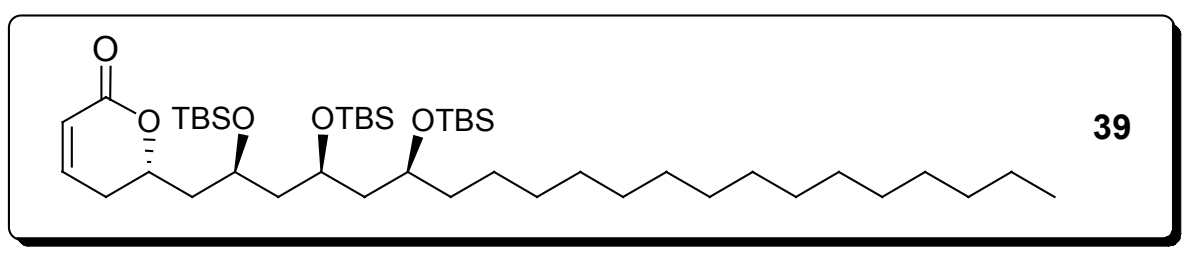

(S)-5,6-Dihydro-6-[(2R,4S,6S)-2,4,6-tris(tert-butyldimethylsilyloxy)henicosyl]pyran-2-one (39): amorphous solid; $[\alpha]_{\mathrm{D}}+6.3\left(c\right.$ 1, $\left.\mathrm{CHCl}_{3}\right) .{ }^{1} \mathrm{H}$ NMR $(500 \mathrm{MHz}) \delta 6.86(\mathrm{~m}, 1 \mathrm{H}), 6.00(\mathrm{dt}, 9.8,1.5 \mathrm{~Hz}$, 1H), $4.60(\mathrm{~m}, 1 \mathrm{H}), 4.18(\mathrm{~m}, 1 \mathrm{H}), 3.83(\mathrm{~m}, 1 \mathrm{H}), 3.72$ (quint, $6 \mathrm{~Hz}, 1 \mathrm{H}), 2.30(\mathrm{~m}, 2 \mathrm{H}), 2.08$ (ddd, 14, 9.7, $2.5 \mathrm{~Hz}, 1 \mathrm{H}), 1.75-1.45$ (br m, 5H), 1.40-1.20 (br m, 28H), $0.89(\mathrm{~s}, 9 \mathrm{H}), 0.88(\mathrm{~s}, 18 \mathrm{H}), 0.87(\mathrm{t}$, overlapped, 3H), $0.11(\mathrm{~s}, 3 \mathrm{H}), 0.10(\mathrm{~s}, 3 \mathrm{H}), 0.07(\mathrm{~s}, 6 \mathrm{H}), 0.05(\mathrm{~s}, 6 \mathrm{H}) .{ }^{13} \mathrm{C} \mathrm{NMR}(125 \mathrm{MHz}) \delta 164.0$, 18.1, 18.0, 17.9 (C), 144.7, 121.8, 74.5, 69.7, 66.8, $65.3(\mathrm{CH}), 46.3,45.8,42.9,37.7,32.0,30.1,29.9$, 29.7 (several overlapped peaks), 29.4, 25.0, $22.7\left(\mathrm{CH}_{2}\right), 26.0$ (x 9), 14.1, -4.1 (x 3), -4.2 (x 2), -4.6 $\left(\mathrm{CH}_{3}\right)$. IR $v_{\max } 1735(\mathrm{C}=\mathrm{O}) \mathrm{cm}^{-1}$. 


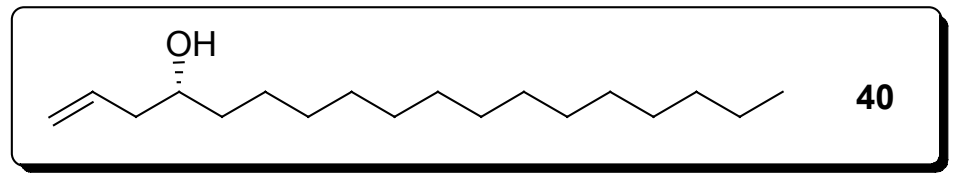

(R)-Octadec-1-en-4-ol (40): solid, mp 47-49 ${ }^{\circ} \mathrm{C}$ (from hexanes); $[\alpha]_{\mathrm{D}}+3.1\left(c\right.$ 1.5, $\left.\mathrm{CHCl}_{3}\right)$. IR and NMR data identical to those of $\mathbf{1 9}$.

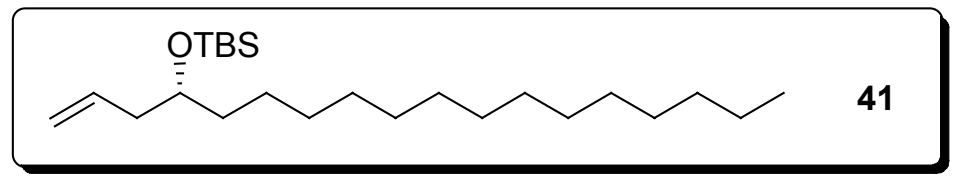

(R)-4-(Tert-butyldimethylsilyloxy)octadec-1-ene (41): oil; $[\alpha]_{\mathrm{D}}+7.7\left(c 2, \mathrm{CHCl}_{3}\right)$. IR and NMR data identical to those of $\mathbf{2 0}$.

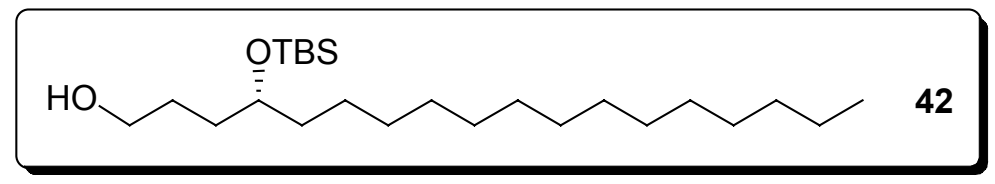

(R)-4-(Tert-butyldimethylsilyloxy)octadecanol (42): oil; $[\alpha]_{\mathrm{D}}-3.2\left(\right.$ c 1.1, $\left.\mathrm{CHCl}_{3}\right)$. IR and NMR data identical to those of $\mathbf{2 1}$.

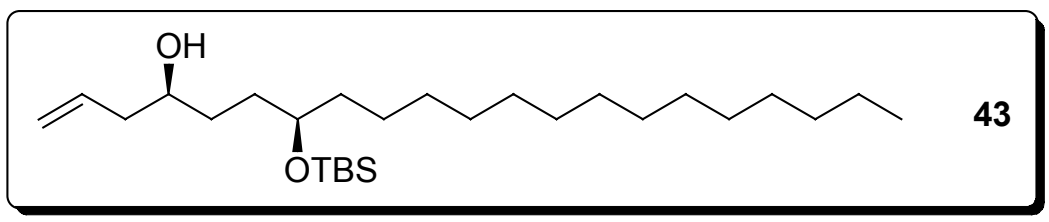

(4S,7R)-7-(Tert-butyldimethylsilyloxy)henicos-1-en-4-ol (43): oil; $[\alpha]_{\mathrm{D}}-3.2\left(c\right.$ 1.6, $\left.\mathrm{CHCl}_{3}\right) .{ }^{1} \mathrm{H} \mathrm{NMR}$ $(500 \mathrm{MHz}) \delta 5.83(\mathrm{~m}, 1 \mathrm{H}), 5.15-5.10(\mathrm{~m}, 2 \mathrm{H}), 3.70-3.60(\mathrm{~m}, 2 \mathrm{H}), 2.30(\mathrm{~m}, 1 \mathrm{H}), 2.18(\mathrm{~m}, 1 \mathrm{H}), 1.80(\mathrm{br}$ $\mathrm{s}, 1 \mathrm{H}, \mathrm{OH}), 1.60-1.50(\mathrm{~m}, 4 \mathrm{H}), 1.45(\mathrm{~m}, 2 \mathrm{H}), 1.35-1.20$ (br m, 24H), 0.89 (s, 9H), 0.88 (t, overlapped, $3 \mathrm{H}) ; 0.05(\mathrm{~s}, 6 \mathrm{H}) .{ }^{13} \mathrm{C}$ NMR $(125 \mathrm{MHz}) \delta 18.1(\mathrm{C}), 135.0,72.2,70.8(\mathrm{CH}), 41.9,36.9,32.5,32.0,29.8$, 29.7 (several overlapped peaks), 29.6, 29.4, 25.4, $22.7\left(\mathrm{CH}_{2}\right), 25.9$ (x 3), 14.1, -4.4 (x 2) $\left(\mathrm{CH}_{3}\right)$. $v_{\max } 3400(\mathrm{br}, \mathrm{OH}) \mathrm{cm}^{-1}$. HR FABMS m/z $423.4045\left(\mathrm{M}+\mathrm{H}^{+}-\mathrm{H}_{2} \mathrm{O}\right)$. Calcd. for $\mathrm{C}_{27} \mathrm{H}_{57} \mathrm{O}_{2} \mathrm{Si}_{-}-\mathrm{H}_{2} \mathrm{O}$, 423.4022 . 


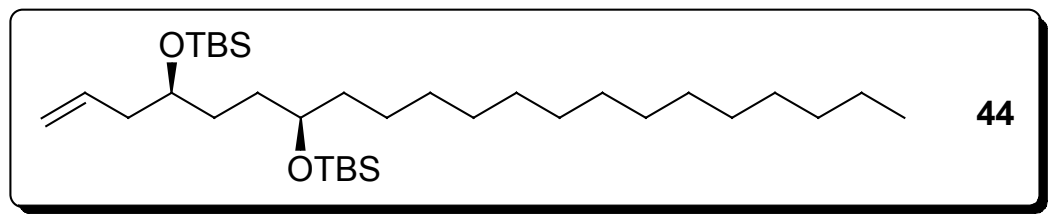

(4S,7R)-4,7-Bis(tert-butyldimethylsilyloxy)henicos-1-ene (44): oil; $[\alpha]_{\mathrm{D}}-2$ (c 1.4, $\left.\mathrm{CHCl}_{3}\right) .{ }^{1} \mathrm{H} \mathrm{NMR}$ $(500 \mathrm{MHz}) \delta 5.83(\mathrm{~m}, 1 \mathrm{H}), 5.15-5.10(\mathrm{~m}, 2 \mathrm{H}), 3.70$ (quint, $6 \mathrm{~Hz}, 1 \mathrm{H}), 3.65$ (quint, $6 \mathrm{~Hz}, 1 \mathrm{H}), 2.20(\mathrm{~m}$, 2H), 1.65-1.40 (m, 4H), 1.35-1.20 (br m, 25H), $0.90(\mathrm{~s}, 18 \mathrm{H}), 0.89$ (t, overlapped, 3H); $0.09(\mathrm{~s}, 3 \mathrm{H})$, 0.08 (s, 3H), 0.07 (s, 3H), $0.06(\mathrm{~s}, 3 \mathrm{H}) .{ }^{13} \mathrm{C} \mathrm{NMR}(125 \mathrm{MHz}) \delta 18.2,18.1(\mathrm{C}), 135.3,72.5,72.3(\mathrm{CH})$, 116.7, 40.1, 37.2, 32.5, 32.0, 29.9, 29.7 (several overlapped peaks), 29.5, 25.4, $22.7\left(\mathrm{CH}_{2}\right), 26.0$ (x 3), 25.9 (x 3), 14.1, -4.3, -4.4 (x 2), -4.5 ( $\left.\mathrm{CH}_{3}\right)$. HR EIMS $m / z$ (\% rel. int.) $497.4127\left(\mathrm{M}^{+}-t \mathrm{Bu}, 25\right), 415$ (24), 381 (100), 365 (56). Calcd. for $\mathrm{C}_{33} \mathrm{H}_{70} \mathrm{O}_{2} \mathrm{Si}_{2}-t \mathrm{Bu}, 497.4204$.

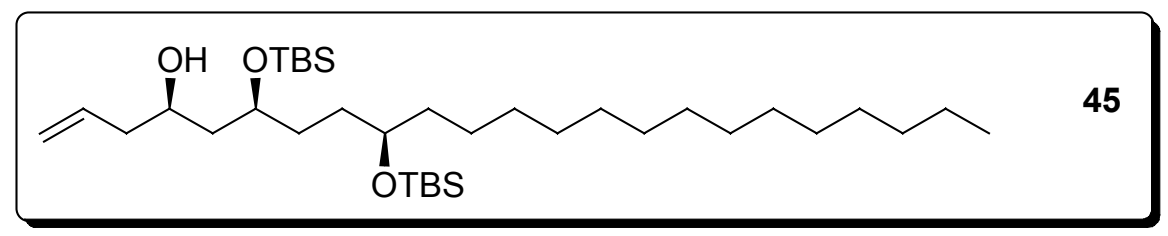

(4R,6S,9R)-6,9-Bis(tert-butyldimethylsilyloxy)tricos-1-en-4-ol (45): oil; $[\alpha]_{\mathrm{D}}+16.2$ (c 1.2, $\left.\mathrm{CHCl}_{3}\right)$. ${ }^{1} \mathrm{H}$ NMR $(500 \mathrm{MHz}) \delta 5.84(\mathrm{~m}, 1 \mathrm{H}), 5.15-5.05(\mathrm{~m}, 2 \mathrm{H}), 3.90(\mathrm{~m}, 1 \mathrm{H}), 3.80(\mathrm{~m}, 1 \mathrm{H}), 3.60$ (quint, $6 \mathrm{~Hz}$, $1 \mathrm{H}), 3.00$ (br s, 1H, OH), 2.23 (t, $6.5 \mathrm{~Hz}, 2 \mathrm{H}), 1.65-1.40(\mathrm{~m}, 6 \mathrm{H}), 1.35-1.20$ (br m, 26H), $0.90(\mathrm{~s}, 9 \mathrm{H})$, $0.88(\mathrm{~s}, 9 \mathrm{H}), 0.88(\mathrm{t}$, overlapped, $3 \mathrm{H}), 0.11(\mathrm{~s}, 3 \mathrm{H}), 0.10(\mathrm{~s}, 3 \mathrm{H}), 0.04(\mathrm{~s}, 6 \mathrm{H}) .{ }^{13} \mathrm{C} \mathrm{NMR}(125 \mathrm{MHz}) \delta$ 18.1, 18.0 (C), 134.9, 73.0, 72.4, $70.0(\mathrm{CH}), 117.4,42.5,42.2,37.3,33.8,32.0,29.9,29.7$ (several overlapped peaks), 29.4, 25.2, $22.7\left(\mathrm{CH}_{2}\right), 25.9$ (x 3), 25.8 (x 3), 14.1, -4.1, -4.4 (x 2), -4.7 ( $\left.\mathrm{CH}_{3}\right) . \mathrm{IR}$ $v_{\max } 3500(\mathrm{br}, \mathrm{OH}) \mathrm{cm}^{-1}$. HR FABMS $m / z 599.5310\left(\mathrm{M}+\mathrm{H}^{+}\right)$. Calcd. for $\mathrm{C}_{35} \mathrm{H}_{75} \mathrm{O}_{3} \mathrm{Si}_{2}, 599.5249$.

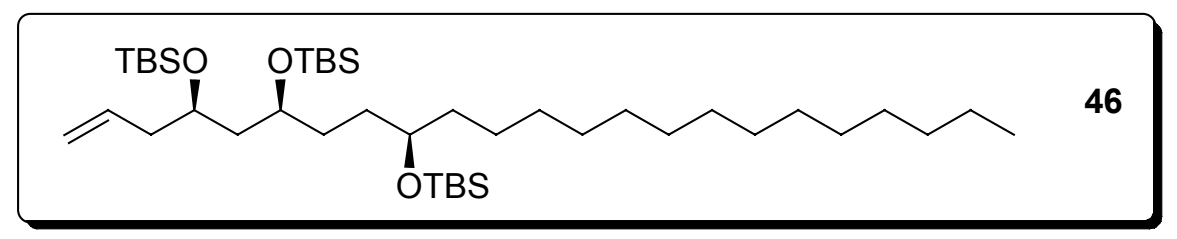

(4R,6S,9R)-4,6,9-Tris(tert-butyldimethylsilyloxy)tricos-1-ene (46): oil; $[\alpha]_{\mathrm{D}}-2$ (c 1.6, $\left.\mathrm{CHCl}_{3}\right) .{ }^{1} \mathrm{H}$ NMR (500 MHz) $\delta 5.83(\mathrm{~m}, 1 \mathrm{H}), 5.15-5.05(\mathrm{~m}, 2 \mathrm{H}), 3.83$ (quint, $6 \mathrm{~Hz}, 1 \mathrm{H}), 3.77$ (quint, $6 \mathrm{~Hz}, 1 \mathrm{H}$ ), 3.62 (quint, $6 \mathrm{~Hz}, 1 \mathrm{H}), 2.28(\mathrm{~m}, 1 \mathrm{H}), 2.18(\mathrm{~m}, 1 \mathrm{H}), 1.65-1.55(\mathrm{~m}, 2 \mathrm{H}), 1.50-1.40(\mathrm{~m}, 2 \mathrm{H}), 1.35-1.20$ (br 
m, 28H), 0.90 (s, 27H), 0.88 (t, overlapped, 3H), 0.09 (s, 3H), $0.08(\mathrm{~s}, 6 \mathrm{H}), 0.07$ (s, 6H), 0.05 (s, 3H). ${ }^{13} \mathrm{C}$ NMR $(125 \mathrm{MHz}) \delta 18.2,18.1,18.0(\mathrm{C}), 135.0,72.7,69.7,69.4(\mathrm{CH}), 117.0,44.6,42.1,37.2,32.9$, 32.0, 29.9, 29.7 (several overlapped peaks), 29.4, 25.3, $22.7\left(\mathrm{CH}_{2}\right), 26.0$ (x 9), 14.1, -4.3 (x 2), -4.4 (x 3), $-4.5\left(\mathrm{CH}_{3}\right)$. HR EIMS $m / z$ (\% rel. int.) $655.5334\left(\mathrm{M}^{+}-t \mathrm{Bu}, 15\right), 523(20), 455(50), 381$ (84), 185 (88), 73 (100). Calcd. for $\mathrm{C}_{41} \mathrm{H}_{88} \mathrm{O}_{3} \mathrm{Si}_{3}-t \mathrm{Bu}, 655.5337$.

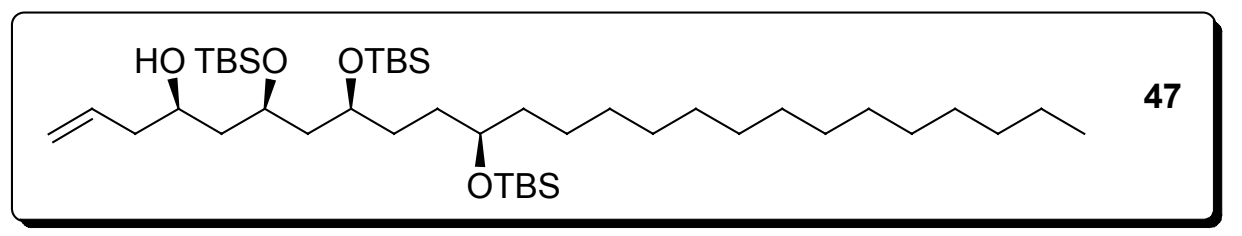

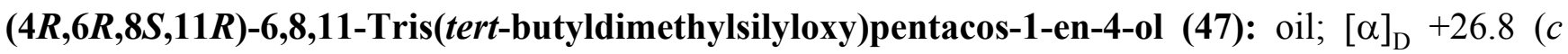
1.9, $\left.\mathrm{CHCl}_{3}\right) .{ }^{1} \mathrm{H}$ NMR $(500 \mathrm{MHz}) \delta 5.83(\mathrm{~m}, 1 \mathrm{H}), 5.15-5.05(\mathrm{~m}, 2 \mathrm{H}), 4.06$ (quint, $\left.6 \mathrm{~Hz}, 1 \mathrm{H}\right), 3.80(\mathrm{~m}$, $1 \mathrm{H}), 3.67$ (m, 1H), 3.60 (quint, $6 \mathrm{~Hz}, 1 \mathrm{H}), 3.30$ (br s, 1H, OH), 2.30-2.20 (m, 2H), 1.80-1.70 (m, 2H), 1.55-1.40 (m, 4H), 1.35-1.20 (br m, 28H), $0.90(\mathrm{~s}, 9 \mathrm{H}), 0.88(\mathrm{~s}, 18 \mathrm{H}), 0.87$ (t, overlapped, 3H), $0.11(\mathrm{~s}$, $6 \mathrm{H}), 0.04(\mathrm{~s}, 9 \mathrm{H}), 0.03$ (s, 3H). ${ }^{13} \mathrm{C} \mathrm{NMR}(125 \mathrm{MHz}) \delta 18.1,18.0,17.9(\mathrm{C}), 135.0,72.5,70.9,70.4$, $69.6(\mathrm{CH}), 117.4,45.7,42.4,42.3,37.2,33.8,32.0,31.9,29.9$, 29.7 (several overlapped peaks), 29.4, 25.2, $22.7\left(\mathrm{CH}_{2}\right), 26.0$ (x 3), 25.9 (x 3), 25.8 (x 3), 14.1, -4.0, -4.1, -4.4 (x 2), -4.5, -4.6 ( $\left.\mathrm{CH}_{3}\right)$. IR $v_{\max } 3500$ (br, OH) $\mathrm{cm}^{-1}$. HR EIMS $m / z$ (\% rel. int.) $699.5695\left(\mathrm{M}^{+}-t \mathrm{Bu}, 1\right), 567$ (27), 435 (55), 381 (74), 341 (47), 73 (100). Calcd. for $\mathrm{C}_{43} \mathrm{H}_{92} \mathrm{O}_{4} \mathrm{Si}_{3}-t \mathrm{Bu}, 699.5599$.

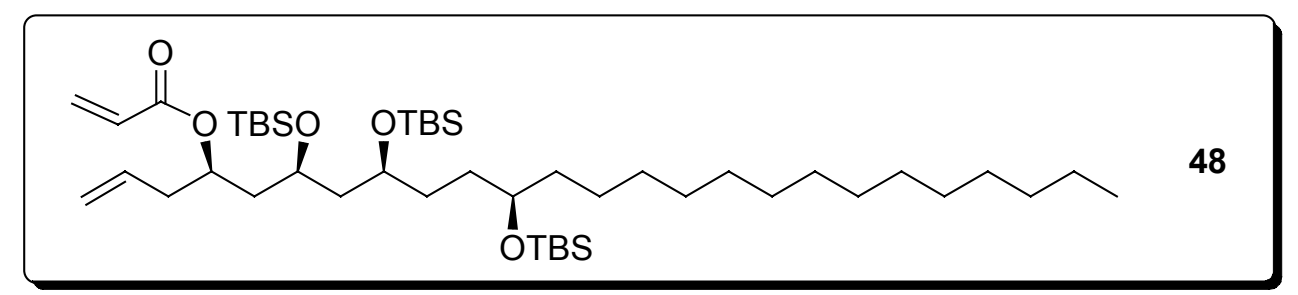

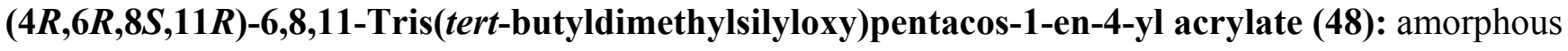
solid; $[\alpha]_{\mathrm{D}}-9.2\left(c\right.$ 2.2, $\left.\mathrm{CHCl}_{3}\right) .{ }^{1} \mathrm{H}$ NMR $(500 \mathrm{MHz}) \delta 6.39(\mathrm{dd}, 17.3,1.5 \mathrm{~Hz}, 1 \mathrm{H}), 6.09(\mathrm{dd}, 17.3,10.5$ $\mathrm{Hz}, 1 \mathrm{H}), 5.80-5.70(\mathrm{~m}, 2 \mathrm{H}), 5.15(\mathrm{~m}, 1 \mathrm{H}), 5.10-5.05(\mathrm{~m}, 2 \mathrm{H}), 3.80(\mathrm{~m}, 1 \mathrm{H}), 3.76(\mathrm{~m}, 1 \mathrm{H}), 3.60(\mathrm{~m}$, 1H), $2.40(\mathrm{~m}, 1 \mathrm{H}), 2.33(\mathrm{~m}, 1 \mathrm{H}), 1.82(\mathrm{ddd}, 14,8,4.7 \mathrm{~Hz}, 1 \mathrm{H}), 1.75$ (ddd, 14, 6.8, $4.7 \mathrm{~Hz}, 1 \mathrm{H}), 1.60$ $(\mathrm{m}, 2 \mathrm{H}), 1.55-1.40(\mathrm{~m}, 2 \mathrm{H}), 1.35-1.20($ br m, 27H) $0.90(\mathrm{~s}, 9 \mathrm{H}), 0.88(\mathrm{~s}, 18 \mathrm{H}), 0.88$ (t, overlapped, $3 \mathrm{H}), 0.07(\mathrm{~s}, 3 \mathrm{H}), 0.05(\mathrm{~s}, 9 \mathrm{H}), 0.04(\mathrm{~s}, 3 \mathrm{H}), 0.03(\mathrm{~s}, 3 \mathrm{H}) .{ }^{13} \mathrm{C}$ NMR $(125 \mathrm{MHz}) \delta 165.4,18.2,18.1$, 18.0 (C), 133.5, 128.8, 72.6, 70.7, 69.5, 66.9 (CH), 130.3, 117.9, 44.8, 41.5, 39.2, 37.0, 32.5, 32.4, 32.0, 29.9, 29.7 (several overlapped peaks), 29.4, 25.3, $22.7\left(\mathrm{CH}_{2}\right), 26.0$ (x 3), 25.9 (x 3), 25.8 (x 3), $14.1,-4.3$ (x 2), -4.4 (x 2), -4.5 (x 2) $\left(\mathrm{CH}_{3}\right)$. IR $v_{\max } 1729(\mathrm{C}=\mathrm{O}) \mathrm{cm}^{-1}$. FABMS m/z $811\left(\mathrm{M}+\mathrm{H}^{+}\right)$. HR 
EIMS $m / z$ (\% rel. int.) $753.5706\left(\mathrm{M}^{+}-t \mathrm{Bu}, 1\right), 381$ (100), 343 (61). Calcd. for $\mathrm{C}_{46} \mathrm{H}_{94} \mathrm{O}_{5} \mathrm{Si}_{3}-t \mathrm{Bu}_{\text {, }}$ 753.5704 .

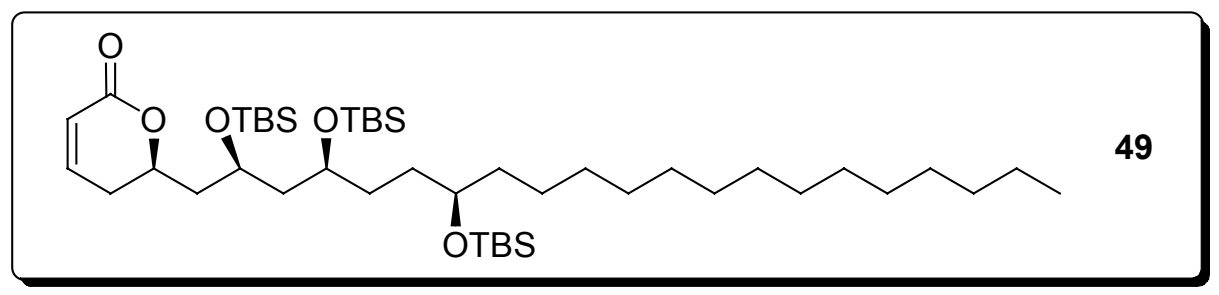

(R)-5,6-Dihydro-6-[(2R,4S,7R)-2,4,7-tris(tert-butyldimethylsilyloxy)henicosyl]pyran-2-one (49): amorphous solid; $\left.[\alpha]_{\mathrm{D}}+26(c), \mathrm{CHCl}_{3}\right) .{ }^{1} \mathrm{H} \mathrm{NMR}(500 \mathrm{MHz}) \delta 6.86(\mathrm{~m}, 1 \mathrm{H}), 6.02(\mathrm{dd}, 9.8,2 \mathrm{~Hz}, 1 \mathrm{H})$, $4.60(\mathrm{~m}, 1 \mathrm{H}), 4.01$ (quint, $6 \mathrm{~Hz}, 1 \mathrm{H}), 3.75(\mathrm{~m}, 1 \mathrm{H}), 3.60$ (quint, $6 \mathrm{~Hz}, 1 \mathrm{H}), 2.40$ (dt, 18, $5 \mathrm{~Hz}, 1 \mathrm{H}), 2.30$ (ddt, 18, 11.5, $2.5 \mathrm{~Hz}, 1 \mathrm{H}), 2.02$ (dt, 14, $6 \mathrm{~Hz}, 1 \mathrm{H}), 1.85$ (dt, 14, $6 \mathrm{~Hz}, 1 \mathrm{H}), 1.70-1.60$ (m, 2H), 1.50$1.40(\mathrm{~m}, 3 \mathrm{H}), 1.35-1.20$ (br m, 27H), 0.89 (s, 18H), $0.88(\mathrm{~s}, 9 \mathrm{H}), 0.88$ (t, overlapped, 3H), $0.08(\mathrm{~s}, 3 \mathrm{H})$, $0.07(\mathrm{~s}, 6 \mathrm{H}), 0.06(\mathrm{~s}, 3 \mathrm{H}), 0.05(\mathrm{~s}, 6 \mathrm{H}) .{ }^{13} \mathrm{C}$ NMR $(125 \mathrm{MHz}) \delta 164.2,18.1,18.0,17.9(\mathrm{C}), 144.7$, 121.7, 75.2, 72.6, 69.5, $66.3(\mathrm{CH}), 44.5,41.8,37.1,33.3,32.3,32.0,29.9,29.7$ (several overlapped peaks), 29.4, 25.3, $22.7\left(\mathrm{CH}_{2}\right), 26.0$ (x 3), 25.9 (x 3), 25.8 (x 3), 14.1, -4.1, -4.3 (x 2), -4.4 (x 2), -4.5 $\left(\mathrm{CH}_{3}\right)$. IR $v_{\max } 1739(\mathrm{C}=\mathrm{O}) \mathrm{cm}^{-1}$. FABMS $m / z 782\left(\mathrm{M}^{+}\right)$.

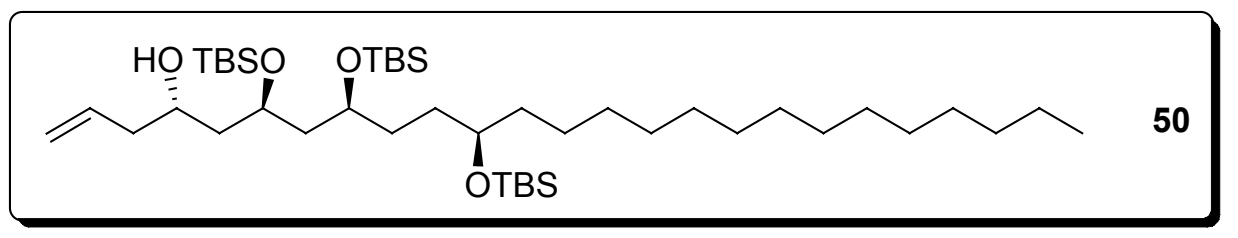

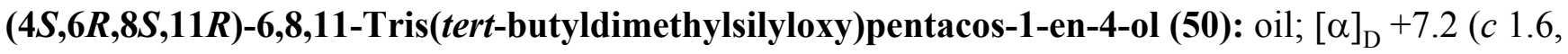
$\left.\mathrm{CHCl}_{3}\right) .{ }^{1} \mathrm{H}$ NMR $(500 \mathrm{MHz}) \delta 5.83(\mathrm{~m}, 1 \mathrm{H}), 5.15-5.05(\mathrm{~m}, 2 \mathrm{H}), 4.18(\mathrm{~m}, 1 \mathrm{H}), 3.98(\mathrm{~m}, 1 \mathrm{H}), 3.60(\mathrm{~m}$, 2H), 3.50 (br s, 1H, OH), 2.27 (m, 1H), $2.17(\mathrm{~m}, 1 \mathrm{H}), 1.80-1.70(\mathrm{~m}, 2 \mathrm{H}), 1.70-1.60(\mathrm{~m}, 2 \mathrm{H}), 1.55$ (m, 1H), 1.35-1.20 (br m, 29H), $0.90(\mathrm{~s}, 9 \mathrm{H}), 0.89(\mathrm{~s}, 9 \mathrm{H}), 0.88(\mathrm{~s}, 9 \mathrm{H}), 0.87$ (t, overlapped, 3H), $0.11(\mathrm{~s}$, $3 \mathrm{H}), 0.10$ (s, 3H), 0.05 (br s, 12H). ${ }^{13} \mathrm{C}$ NMR (125 MHz) $\delta 18.1,18.0,17.9$ (C), 135.0, 72.5, 69.8, 69.2, $67.8(\mathrm{CH}), 117.3,43.2,42.4,40.5,37.2,33.7,32.2,31.9,29.9,29.7$ (several overlapped peaks), 29.4, 25.3, $22.7\left(\mathrm{CH}_{2}\right), 26.0$ (x 3), 25.9 (x 3), 25.8 (x 3), 14.1, -4.1, -4.4 (x 2), -4.6 (x 2), -4.7 ( $\left.\mathrm{CH}_{3}\right)$. IR $v_{\max } 3500(\mathrm{br}, \mathrm{OH}) \mathrm{cm}^{-1}$. HR EIMS $m / z$ (\% rel. int.) $699.5555\left(\mathrm{M}^{+}-t \mathrm{Bu}, 1\right), 567$ (27), 435 (50), 381 (44), 341 (37), 73 (100). Calcd. for $\mathrm{C}_{43} \mathrm{H}_{92} \mathrm{O}_{4} \mathrm{Si}_{3}-t \mathrm{Bu}, 699.5599$. 


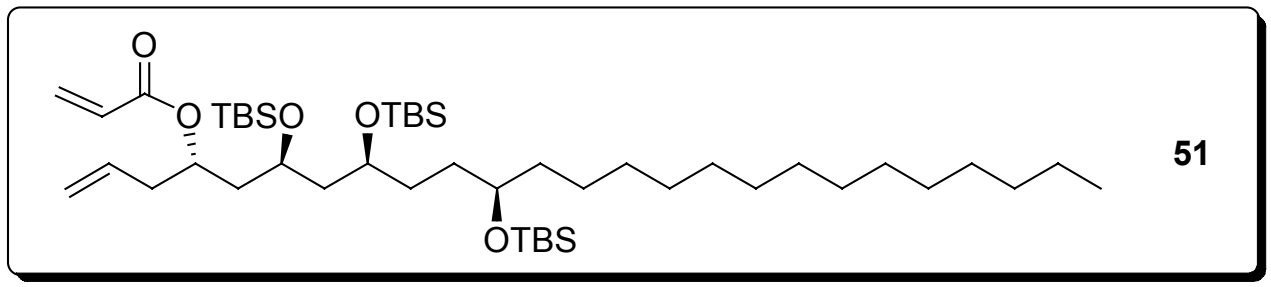

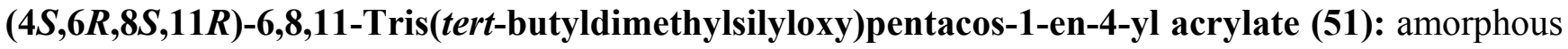
solid; $[\alpha]_{\mathrm{D}}+24.4\left(c\right.$ 1.9, $\left.\mathrm{CHCl}_{3}\right) .{ }^{1} \mathrm{H}$ NMR $(500 \mathrm{MHz}) \delta 6.39(\mathrm{dd}, 17.3,1.5 \mathrm{~Hz}, 1 \mathrm{H}), 6.09(\mathrm{dd}, 17.3$, $10.5 \mathrm{~Hz}, 1 \mathrm{H}), 5.80-5.70(\mathrm{~m}, 2 \mathrm{H}), 5.10(\mathrm{~m}, 1 \mathrm{H}), 5.10-5.05(\mathrm{~m}, 2 \mathrm{H}), 3.89(\mathrm{~m}, 1 \mathrm{H}), 3.70(\mathrm{~m}, 1 \mathrm{H}), 3.60$ (quint, $5.5 \mathrm{~Hz}, 1 \mathrm{H}), 2.40(\mathrm{~m}, 2 \mathrm{H}), 1.88(\mathrm{ddd}, 14.5,9.5,3 \mathrm{~Hz}, 1 \mathrm{H}), 1.72$ (ddd, 14.5, 7.5, $4.5 \mathrm{~Hz}, 1 \mathrm{H})$, 1.60-1.40 (m, 4H), 1.35-1.20 (br m, 28H), 0.90 (s, 18H), $0.88(\mathrm{~s}, 9 \mathrm{H}), 0.88$ (t, overlapped, 3H), 0.07 (s, $3 \mathrm{H}), 0.06(\mathrm{~s}, 3 \mathrm{H}), 0.05(\mathrm{~s}, 6 \mathrm{H}), 0.04(\mathrm{~s}, 3 \mathrm{H}), 0.00(\mathrm{~s}, 3 \mathrm{H}) .{ }^{13} \mathrm{C} \mathrm{NMR}(125 \mathrm{MHz}) \delta 165.7,18.2,18.1$ (x 2)(C), 133.5, 129.0, 72.6, 71.0, 69.5, $66.4(\mathrm{CH}), 130.2,117.9,45.8,41.2,39.1,37.2,33.6,32.2,32.0$, 29.9, 29.7 (several overlapped peaks), 29.4, 25.3, $22.7\left(\mathrm{CH}_{2}\right), 26.0$ (x 9), 14.1, -4.1, -4.2, -4.3 (x 3), $-4.7\left(\mathrm{CH}_{3}\right)$. IR $v_{\max } 1729(\mathrm{C}=\mathrm{O}) \mathrm{cm}^{-1}$. FABMS $m / z 811\left(\mathrm{M}+\mathrm{H}^{+}\right)$. HR EIMS $m / z$ (\% rel. int.) 753.5794 $\left(\mathrm{M}^{+}-t \mathrm{Bu}, 1\right), 381$ (100), 343 (41). Calcd. for $\mathrm{C}_{46} \mathrm{H}_{94} \mathrm{O}_{5} \mathrm{Si}_{3}-t \mathrm{Bu}, 753.5704$.

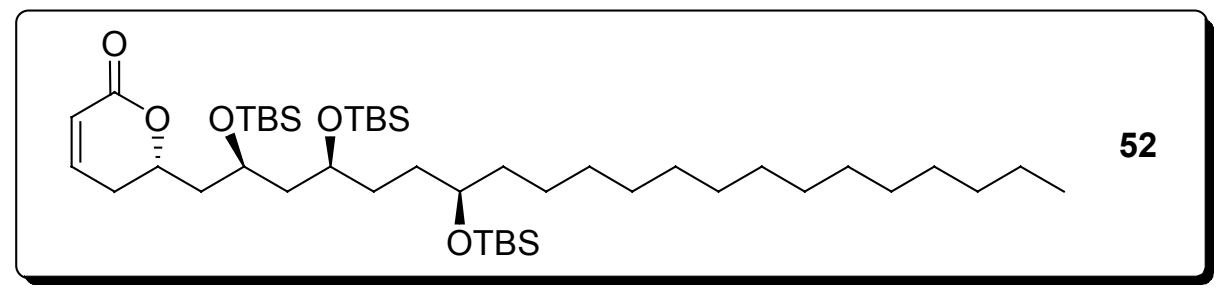

(S)-5,6-Dihydro-6-[(2R,4S,7R)-2,4,7-tris(tert-butyldimethylsilyloxy)henicosyl]pyran-2-one $\quad(52):$ amorphous solid; $[\alpha]_{\mathrm{D}}+4\left(c\right.$ 2.2, $\left.\mathrm{CHCl}_{3}\right) .{ }^{1} \mathrm{H}$ NMR $(500 \mathrm{MHz}) \delta 6.86(\mathrm{dt}, 9.8,4.2 \mathrm{~Hz}, 1 \mathrm{H}), 6.01(\mathrm{dt}$, 9.8, $2 \mathrm{~Hz}, 1 \mathrm{H}), 4.60(\mathrm{~m}, 1 \mathrm{H}), 4.15(\mathrm{~m}, 1 \mathrm{H}), 3.73(\mathrm{~m}, 1 \mathrm{H}), 3.60$ (quint, $6 \mathrm{~Hz}, 1 \mathrm{H}), 2.30(\mathrm{~m}, 2 \mathrm{H}), 2.02$ (ddd, 14, 9.5, $2.5 \mathrm{~Hz}, 1 \mathrm{H}), 1.73$ (ddd, 14, 7.5, 4.2 Hz, 1H), 1.65-1.40 (m, 5H), 1.35-1.20 (br m, 27H), $0.90(\mathrm{~s}, 9 \mathrm{H}), 0.89(\mathrm{~s}, 9 \mathrm{H}), 0.88(\mathrm{~s}, 9 \mathrm{H}), 0.88(\mathrm{t}$, overlapped, 3H), $0.10(\mathrm{~s}, 3 \mathrm{H}), 0.09(\mathrm{~s}, 3 \mathrm{H}), 0.07(\mathrm{~s}, 3 \mathrm{H})$, 0.06 (s, 3H), 0.05 (s, 6H). ${ }^{13} \mathrm{C}$ NMR (125 MHz) $\delta 164.0,18.1,18.0$ (x 2) (C), 144.8, 121.7, 74.5, 72.5, 69.4, $65.5(\mathrm{CH}), 45.7,42.8,37.3,33.7,32.4,32.0,30.2,29.9,29.7$ (several overlapped peaks), 29.4, 25.3, $22.7\left(\mathrm{CH}_{2}\right), 26.0$ (x 9), 14.1, -4.2 (x 2), -4.3, -4.4 (x 2), -4.7 $\left(\mathrm{CH}_{3}\right)$. IR $v_{\max } 1739(\mathrm{C}=\mathrm{O}) \mathrm{cm}^{-1}$. FABMS $m / z 782\left(\mathrm{M}^{+}\right)$. 


\section{Analytical data of target lactones (including NMR spectra) and derivatives}

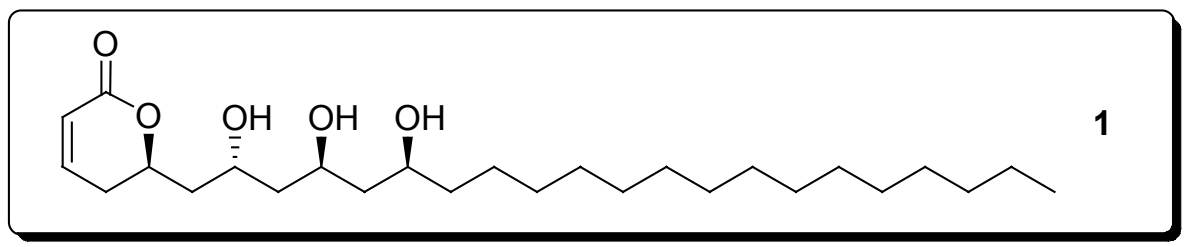

(R)-5,6-Dihydro-6-[(2R,4S,6S)-2,4,6-trihydroxyhenicosyl]pyran-2-one (1): colorless solid, mp 103$105^{\circ} \mathrm{C}$ (from EtOAc-MeOH); $[\alpha]_{\mathrm{D}}+16.7$ (c 1.3; MeOH); $[\alpha]_{\mathrm{D}}+28.1$ (c 1.3; $\left.\mathrm{CHCl}_{3}\right) .{ }^{1} \mathrm{H}$ NMR $(500$ MHz) $\delta 6.87$ (ddd, 9.8, 5, 3.5 Hz, 1H), 5.98 (br d, 9.8 Hz, 1H), 4.74 (br t, 9.7 Hz, 1H), 4.50 (br s, $1 \mathrm{H}$, $\mathrm{OH}), 4.26(\mathrm{~m}, 1 \mathrm{H}), 4.16(\mathrm{~m}, 1 \mathrm{H}), 4.00$ (br s, 1H, OH), $3.82(\mathrm{~m}, 1 \mathrm{H}), 3.50$ (br s, 1H, OH), $2.35(\mathrm{~m}, 2 \mathrm{H})$, $1.82(\mathrm{~m}, 1 \mathrm{H}), 1.72(\mathrm{~m}, 1 \mathrm{H}), 1.65-1.30$ (br m, 8H), 1.30-1.20 (br m, 24H), 0.85 (t, $7 \mathrm{~Hz}, 3 \mathrm{H}) ;{ }^{13} \mathrm{C} \mathrm{NMR}$ $(125 \mathrm{MHz}) \delta 164.9(\mathrm{C}), 145.7,121.2,75.1,73.1,70.3,64.1(\mathrm{CH}), 43.6,42.7,42.6,38.3,31.9,30.0$, 29.7 (several overlapped peaks), 29.3, 25.4, $22.7\left(\mathrm{CH}_{2}\right), 14.1\left(\mathrm{CH}_{3}\right)$. IR $v_{\max } 3300$ (br, OH), 1720 (br, $\mathrm{C}=\mathrm{O}) \mathrm{cm}^{-1}$. HR EIMS m/z (\% rel. int.) $441.3580\left(\mathrm{M}+\mathrm{H}^{+}, 1\right), 422$ (2), 404 (5), 193 (100), 141 (66). Calcd. for $\mathrm{C}_{26} \mathrm{H}_{49} \mathrm{O}_{5}$, 441.3580. Anal. Calcd. for $\mathrm{C}_{26} \mathrm{H}_{48} \mathrm{O}_{5}$ : C, 70.87; H, 10.98. Found, C, 71.00; H, 11.11 .

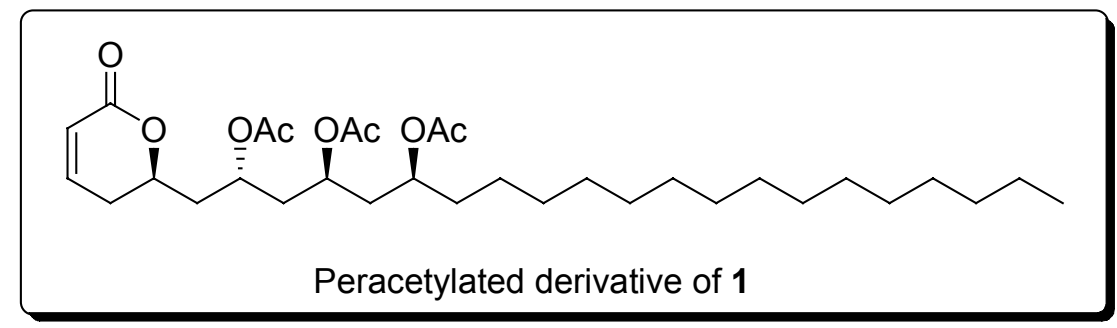

Oil; $[\alpha]_{\mathrm{D}}+13.2\left(c\right.$ 1.8; $\left.\mathrm{CHCl}_{3}\right) .{ }^{1} \mathrm{H}$ NMR $(400 \mathrm{MHz}) \delta 6.84(\mathrm{ddd}, 9.8,5.5,3 \mathrm{~Hz}, 1 \mathrm{H}), 6.00(\mathrm{ddd}, 9.8$, 2.5, $1.2 \mathrm{~Hz}, 1 \mathrm{H}), 5.11$ (hept, $4.5 \mathrm{~Hz}, 1 \mathrm{H}), 4.93$ (m, 1H), 4.87 (m, 1H), 4.45 (m, 1H), 2.35 (m, 2H), 2.03 (s, 3H), 2.01 (s, 3H), 2.00 (s, 3H), 1.98 (ddd, 14, 8.5, 4.5 Hz, 1H), 1.95-1.85 (br m, 4H), 1.70 (m, 2H), 1.52 (br m, 2H), 1.30-1.20 (br m, 25H), 0.86 (t, $7 \mathrm{~Hz}, 3 \mathrm{H}) ;{ }^{13} \mathrm{C}$ NMR (100 MHz) $\delta 170.7,170.5,170.4$, 163.7 (C), 144.6, 121.6, 74.8, 71.0, 67.3, 67.1 (CH), 40.3, 39.3, 39.0, 34.4, 31.9, 29.7 (several overlapped peaks), 29.6, 29.5, 29.4, 29.3, 25.2, $22.7\left(\mathrm{CH}_{2}\right), 21.2$ (two carbons), 21.0, $14.1\left(\mathrm{CH}_{3}\right) . \mathrm{IR}$ $v_{\max } 1735(\mathrm{br}, \mathrm{C}=\mathrm{O}) \mathrm{cm}^{-1}$. 


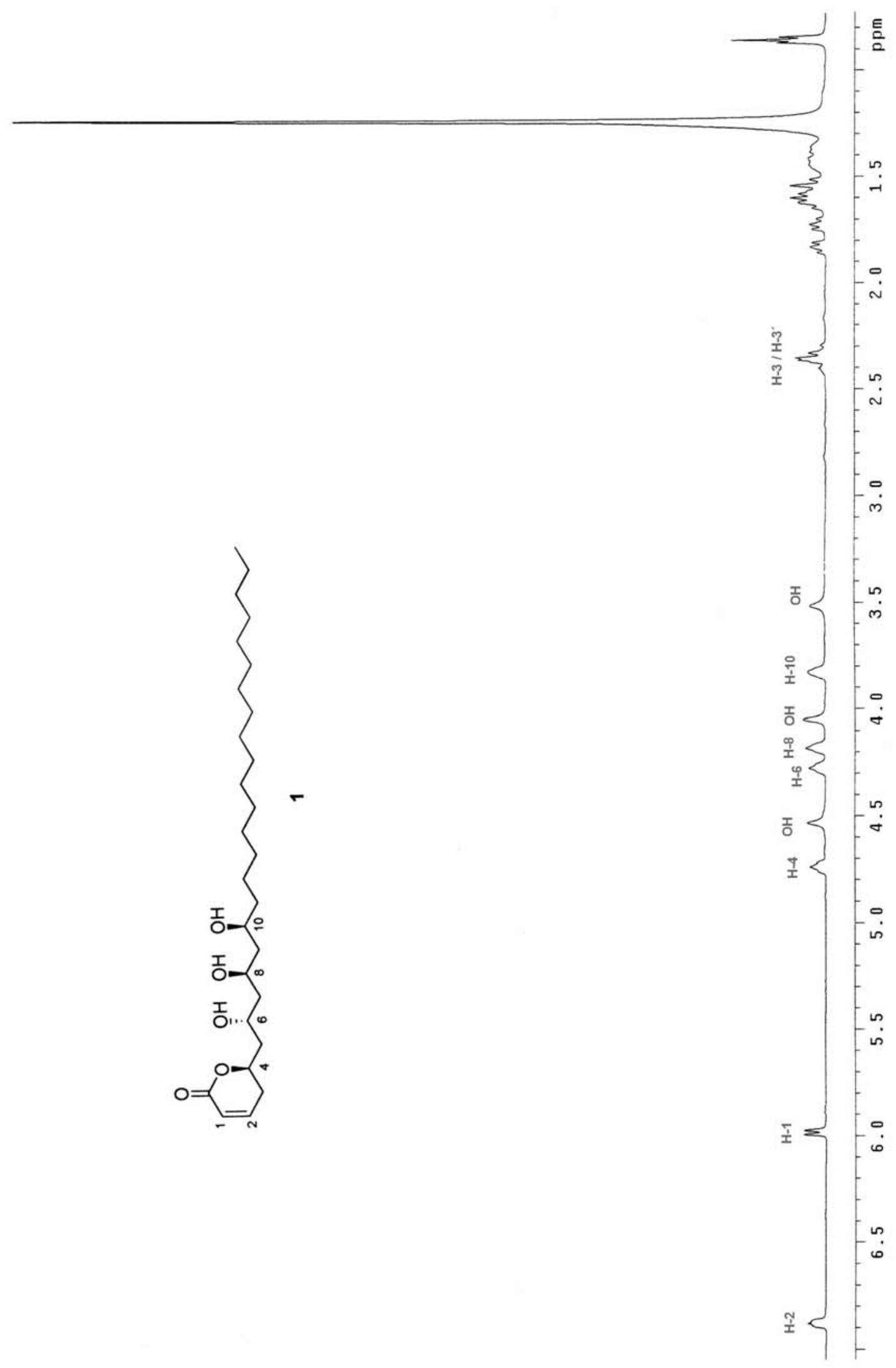




$$
14
$$




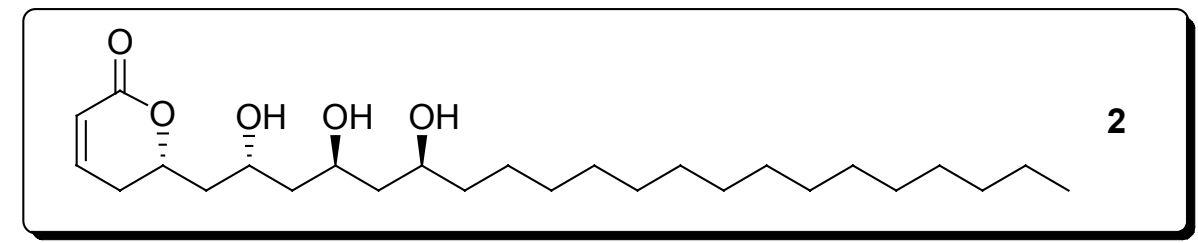

(S)-5,6-Dihydro-6-[(2R,4S,6S)-2,4,6-trihydroxyhenicosyl]pyran-2-one (2): colorless solid, mp 113$114^{\circ} \mathrm{C}$ (from EtOAc-MeOH); $[\alpha]_{\mathrm{D}}-15.8$ (c 1.4; MeOH). ${ }^{1} \mathrm{H}$ NMR (500 MHz) $\delta 6.89$ (ddd, 9.6, 5, 3.5 $\mathrm{Hz}, 1 \mathrm{H}), 6.00$ (br d, $9.6 \mathrm{~Hz}, 1 \mathrm{H}), 4.68$ (m, 1H), 4.40 (br s, 1H, OH), 4.19 (m, 2H), 3.87 (m, 1H), 3.75 (br s, 1H, OH), $2.44(\mathrm{~m}, 2 \mathrm{H}), 2.04(\mathrm{~m}, 1 \mathrm{H}), 1.80-1.30$ (br m, 10H), 1.30-1.20 (br m, 24H), 0.87 (t, 7 $\mathrm{Hz}, 3 \mathrm{H}) ;{ }^{13} \mathrm{C}$ NMR $(125 \mathrm{MHz}) \delta 164.4(\mathrm{C}), 145.6,121.2,76.7,73.1,70.2,65.8(\mathrm{CH}), 43.2,42.6,42.0$, 38.4, 31.9, 29.7 (several overlapped peaks), 29.5, 29.4, 29.3, 25.4, $22.7\left(\mathrm{CH}_{2}\right), 14.1\left(\mathrm{CH}_{3}\right)$. IR $v_{\max }$ 3350 (br, OH), 1720 (br, C=O) cm c $^{-1}$ HR EIMS m/z (\% rel. int.) $441.3567\left(\mathrm{M}+\mathrm{H}^{+}, 1\right), 422$ (6), 404 (8), 193 (88), 141 (100). Calcd. for $\mathrm{C}_{26} \mathrm{H}_{49} \mathrm{O}_{5}, 441.3580$. Anal. Calcd. for $\mathrm{C}_{26} \mathrm{H}_{48} \mathrm{O}_{5}$ : C, 70.87; H, 10.98. Found, C, 70.80; H, 11.09.

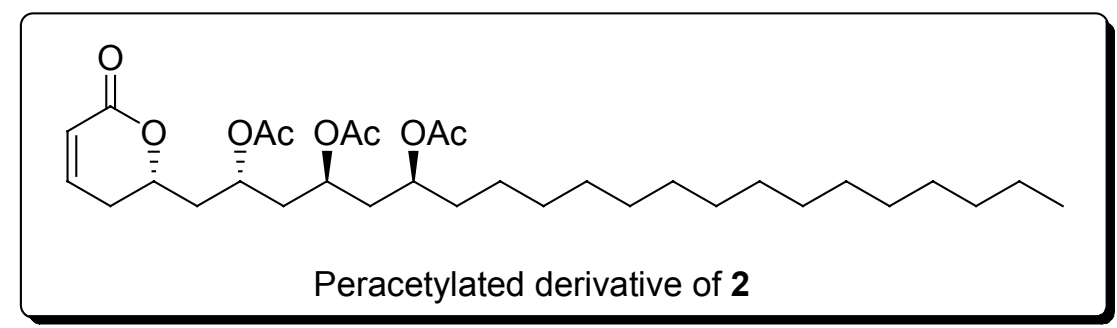

Oil; $[\alpha]_{\mathrm{D}}-15.6\left(c\right.$ 1.6; $\left.\mathrm{CHCl}_{3}\right) .{ }^{1} \mathrm{H}$ NMR $(400 \mathrm{MHz}) \delta 6.85(\mathrm{ddd}, 9.7,6.2,2.3 \mathrm{~Hz}, 1 \mathrm{H}), 6.00$ (br dd, 9.7, $2.5 \mathrm{~Hz}, 1 \mathrm{H}), 5.08(\mathrm{~m}, 1 \mathrm{H}), 4.95(\mathrm{~m}, 1 \mathrm{H}), 4.88(\mathrm{~m}, 1 \mathrm{H}), 4.46(\mathrm{~m}, 1 \mathrm{H}), 2.48(\mathrm{ddd}, 18.2,6.2,3.8 \mathrm{~Hz}, 1 \mathrm{H})$, 2.28 (ddt, 18.2, 11.6, $2.5 \mathrm{~Hz}, 1 \mathrm{H}), 2.13$ (ddd, 14.5, 8, $6.3 \mathrm{~Hz}, 1 \mathrm{H}), 2.04$ (s, 6H), 2.02 (s, 3H), 2.00-1.70 (br m, 6H), 1.52 (br m, 2H), 1.30-1.20 (br m, 25H), 0.87 (t, $7 \mathrm{~Hz}, 3 \mathrm{H}$ ); ${ }^{13} \mathrm{C}$ NMR (100 MHz) $\delta 170.8$, 170.7, 170.5, 163.8 (C), 144.7, 121.5, 74.9, 71.0, 67.2, $66.6(\mathrm{CH}), 40.0,39.3,38.8,34.5,32.0,29.7$ (several overlapped peaks), 29.6, 29.5, 29.4, 29.3, 25.2, $22.7\left(\mathrm{CH}_{2}\right), 21.2,21.1,21.0,14.1\left(\mathrm{CH}_{3}\right) . \mathrm{IR}$ $v_{\max } 1735(\mathrm{br}, \mathrm{C}=\mathrm{O}) \mathrm{cm}^{-1}$. 


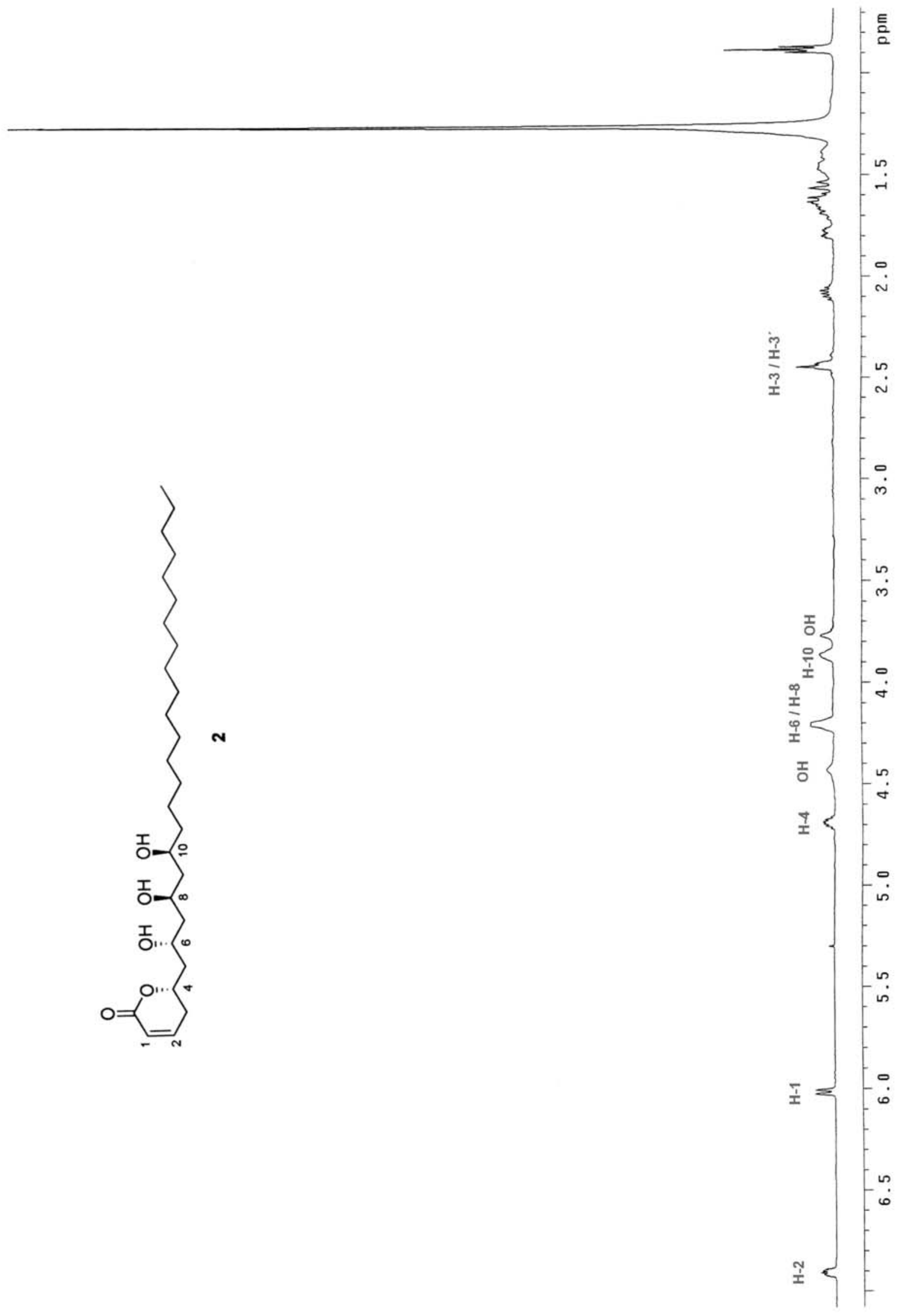




$$
17
$$




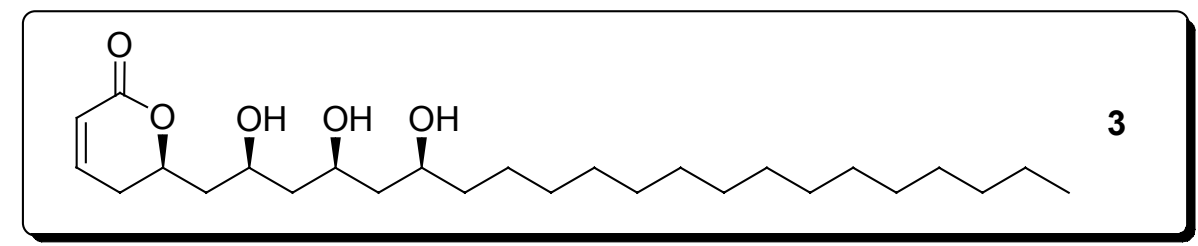

(R)-5,6-Dihydro-6-[(2S,4S,6S)-2,4,6-trihydroxyhenicosyl]pyran-2-one (3): colorless solid, mp 100$101^{\circ} \mathrm{C}$ (from EtOAc-MeOH), lit. mp $101^{\circ} \mathrm{C} ;[\alpha]_{\mathrm{D}}+19.3(c 1.5 ; \mathrm{MeOH}) ;[\alpha]_{\mathrm{D}}+27.4\left(c 1.7 ; \mathrm{CHCl}_{3}\right) .{ }^{1} \mathrm{H}$ NMR (500 MHz) $\delta 6.88(\mathrm{ddd}, 9.8,5.5,3 \mathrm{~Hz}, 1 \mathrm{H}), 6.00(\mathrm{br} \mathrm{d}, 9.8 \mathrm{~Hz}, 1 \mathrm{H}), 4.68(\mathrm{~m}, 1 \mathrm{H}), 4.50$ (br s, $1 \mathrm{H}$, $\mathrm{OH}), 4.14(\mathrm{~m}, 2 \mathrm{H}), 4.00(\mathrm{br} \mathrm{s}, 1 \mathrm{H}, \mathrm{OH}), 3.86(\mathrm{~m}, 1 \mathrm{H}), 2.80(\mathrm{br} \mathrm{s}, 1 \mathrm{H}, \mathrm{OH}), 2.43(\mathrm{~m}, 2 \mathrm{H}), 2.00(\mathrm{~m}, 1 \mathrm{H})$, 1.80-1.40 (br m, 6H), 1.40-1.20 (br m, 27H), 0.87 (t, $7 \mathrm{~Hz}, 3 \mathrm{H}) ;{ }^{13} \mathrm{C}$ NMR $(125 \mathrm{MHz}) \delta 164.3(\mathrm{C})$, 145.4, 121.3, 76.2, 73.6, 73.2, $69.3(\mathrm{CH}), 43.5,43.4,42.3,38.4,31.9,29.7$ (several overlapped peaks), 29.4, 25.3, $22.7\left(\mathrm{CH}_{2}\right)$, $14.1\left(\mathrm{CH}_{3}\right)$. IR $v_{\max } 3300$ (br, OH), $1720(\mathrm{br}, \mathrm{C}=\mathrm{O}) \mathrm{cm}^{-1}$. Anal. Calcd. for $\mathrm{C}_{26} \mathrm{H}_{48} \mathrm{O}_{5}$ : C, 70.87; H, 10.98. Found, C, 71.03; H, 10.88.

Herz, W.; Ramakrishnan, G. Phytochemistry 1978, 17, 1327-1332.

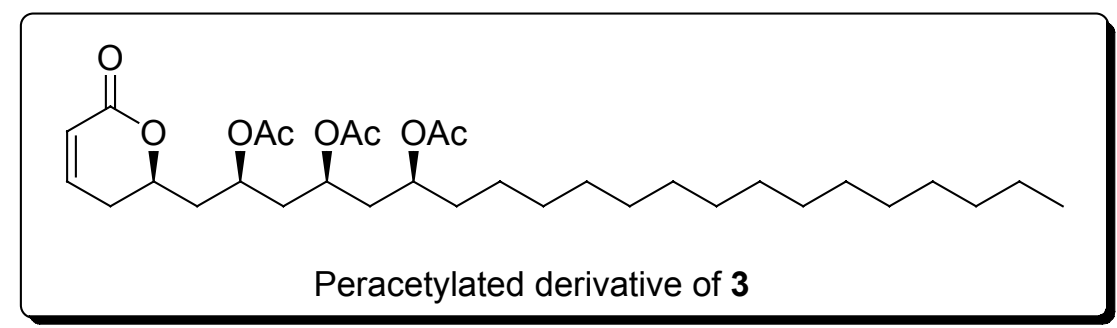

Oil; $[\alpha]_{\mathrm{D}}+26.7\left(c\right.$ 1.5; $\left.\mathrm{CHCl}_{3}\right) .{ }^{1} \mathrm{H}$ NMR $(500 \mathrm{MHz}) \delta 6.85(\mathrm{~m}, 1 \mathrm{H}), 6.00(\mathrm{br} \mathrm{d}, 9.7 \mathrm{~Hz}, 1 \mathrm{H}), 5.05(\mathrm{~m}$, 1H), 4.96 (quint, $6 \mathrm{~Hz}, 1 \mathrm{H}), 4.89$ (quint, $6 \mathrm{~Hz}, 1 \mathrm{H}), 4.47$ (m, 1H), 2.44 (br d, $18 \mathrm{~Hz}, 1 \mathrm{H}), 2.30$ (br dd, 18, $11.5 \mathrm{~Hz}, 1 \mathrm{H}), 2.13(\mathrm{~m}, 1 \mathrm{H}), 2.06(\mathrm{~s}, 3 \mathrm{H}), 2.05(\mathrm{~s}, 3 \mathrm{H}), 2.04(\mathrm{~s}, 3 \mathrm{H}), 2.00-1.85$ (br m, 3H), $1.80(\mathrm{~m}$, 2H), 1.50 (m, 1H), 1.30-1.20 (br m, 27H), 0.87 (t, $7 \mathrm{~Hz}, 3 \mathrm{H}) ;{ }^{13} \mathrm{C} \mathrm{NMR}(125 \mathrm{MHz}) \delta 170.7,170.6$, 170.5, 163.7 (C), 144.7, 121.4, 74.9, 71.0, 68.3, $67.7(\mathrm{CH}), 39.1,38.9$, 38.7, 34.2, 31.9, 29.7 (several overlapped peaks), 29.6, 29.5, 29.4, 29.3, 29.1, 25.1, $22.7\left(\mathrm{CH}_{2}\right), 21.2,21.1,21.0,14.1\left(\mathrm{CH}_{3}\right)$. IR $v_{\max }$ 1735 (br, $\mathrm{C}=\mathrm{O}) \mathrm{cm}^{-1}$. 


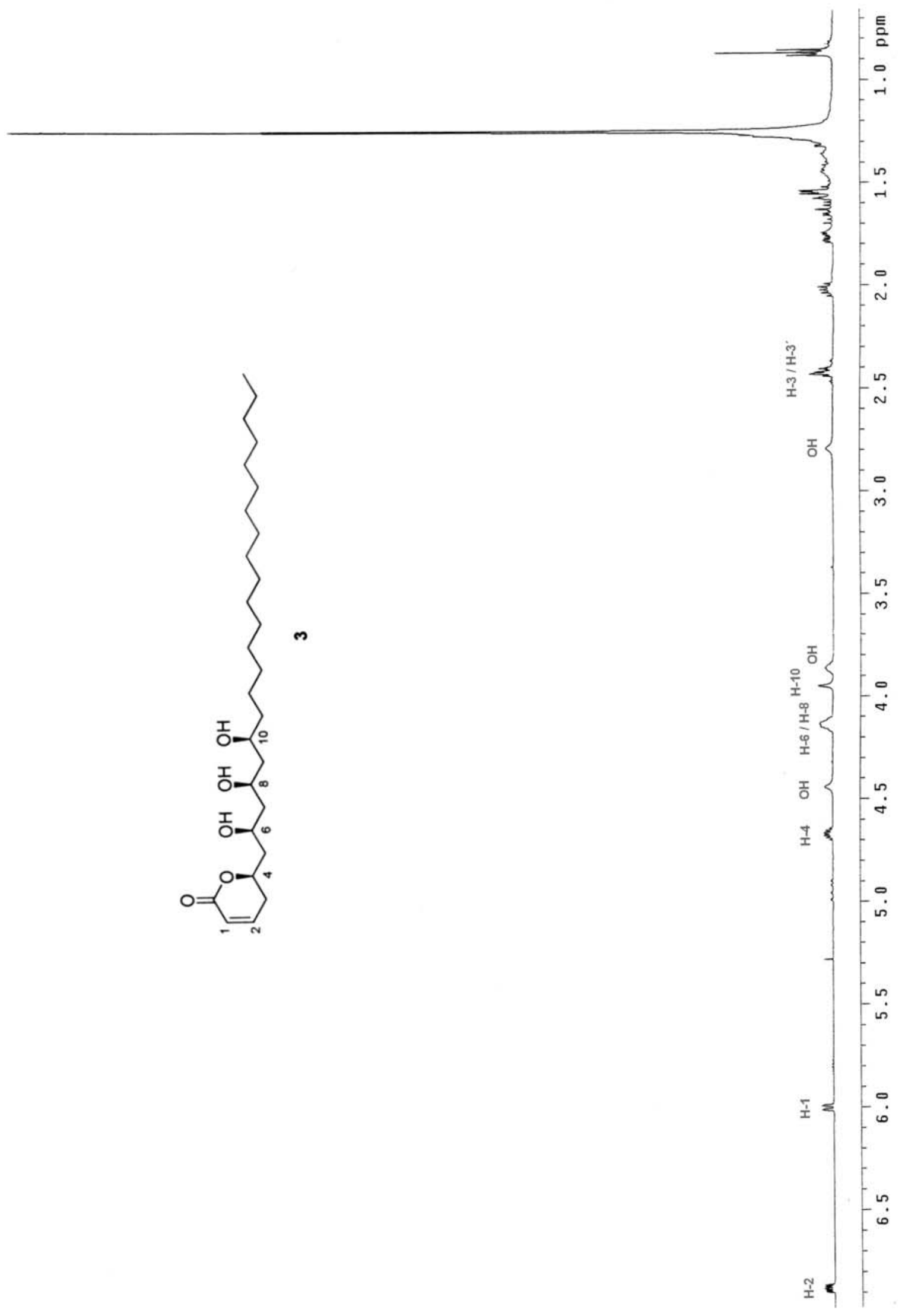

S-31 


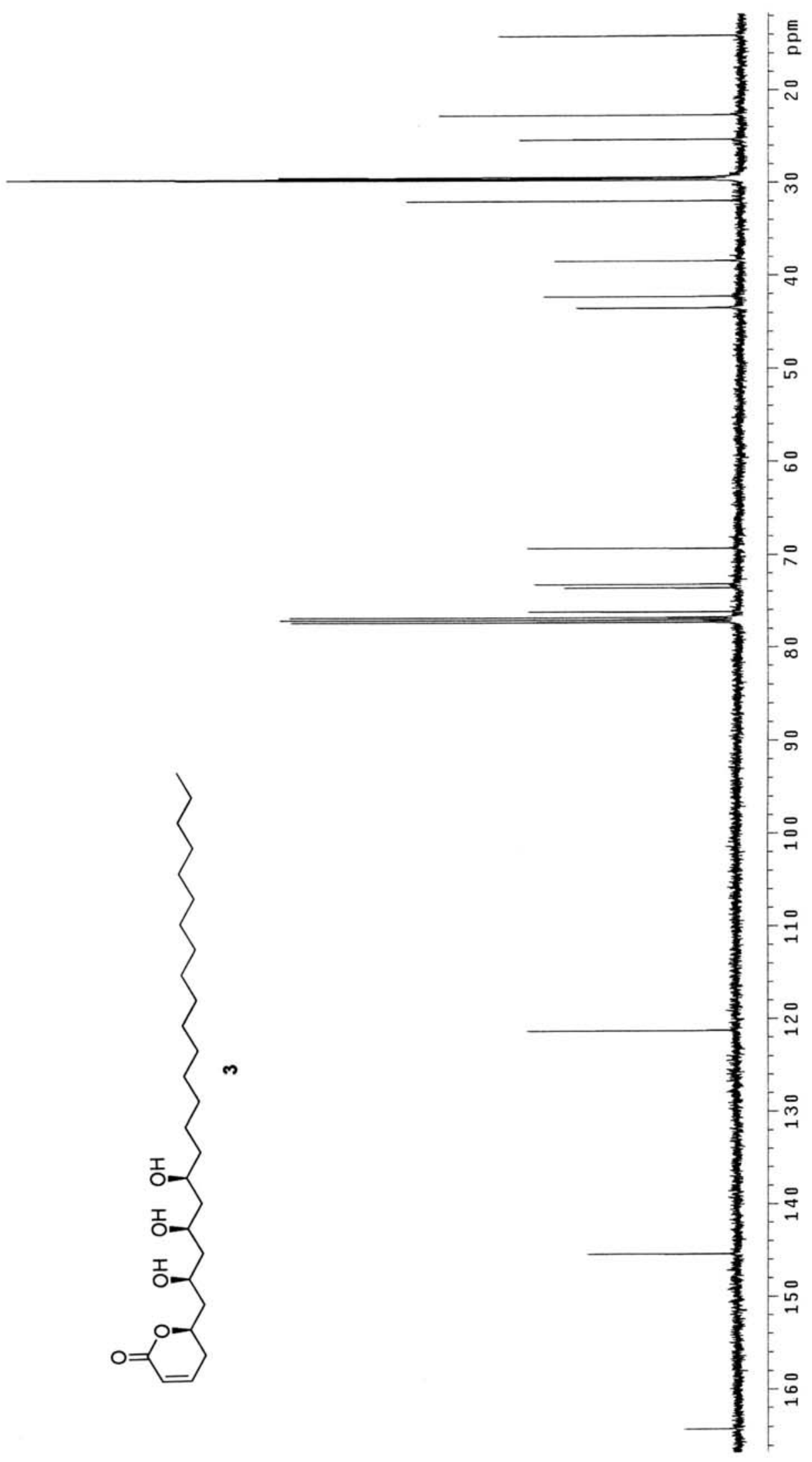

S-32 


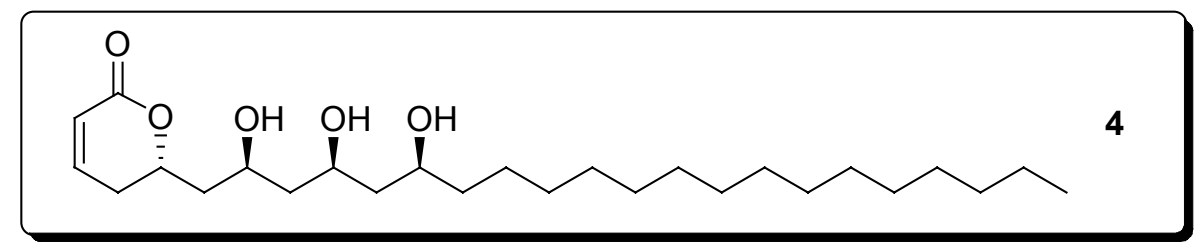

(S)-5,6-Dihydro-6-[(2S,4S,6S)-2,4,6-trihydroxyhenicosyl]pyran-2-one (4): colorless solid, mp 99$100^{\circ} \mathrm{C}$ (from EtOAc-MeOH); $[\alpha]_{\mathrm{D}}-14.4(c$ 1.4; $\mathrm{MeOH}) ;[\alpha]_{\mathrm{D}}-23.5\left(c\right.$ 1.7; $\left.\mathrm{CHCl}_{3}\right) .{ }^{1} \mathrm{H}$ NMR $(500$ MHz) $\delta 6.88(\mathrm{ddd}, 9.8,5.7,2.8 \mathrm{~Hz}, 1 \mathrm{H}), 6.00(\mathrm{dd}, 9.8,2.8 \mathrm{~Hz}, 1 \mathrm{H}), 4.73(\mathrm{~m}, 1 \mathrm{H}), 4.40$ (br s, 1H, OH), 4.24 (tt, 9.6, $2.5 \mathrm{~Hz}, 1 \mathrm{H}), 4.15(\mathrm{~m}, 1 \mathrm{H}), 4.00$ (br s, $1 \mathrm{H}, \mathrm{OH}), 3.88(\mathrm{~m}, 1 \mathrm{H}), 2.60$ (br s, 1H, OH), 2.402.30 (m, 2H), 1.85 (ddd, 14, 9.3, $2.5 \mathrm{~Hz}, 1 \mathrm{H}), 1.70$ (ddd, 14, 9.6, $3 \mathrm{~Hz}, 1 \mathrm{H}), 1.60-1.40$ (br m, 4H), 1.401.20 (br m, 28H), 0.87 (t, $7 \mathrm{~Hz}, 3 \mathrm{H}) ;{ }^{13} \mathrm{C}$ NMR (125 MHz) $\delta 164.5$ (C), 145.3, 121.4, 74.9, 73.7, 73.3, $68.0(\mathrm{CH}), 44.1,43.5,43.1,38.5,31.9,30.0,29.7$ (several overlapped peaks), 29.4, 25.3, $22.7\left(\mathrm{CH}_{2}\right)$, $14.1\left(\mathrm{CH}_{3}\right)$. IR $v_{\max } 3350(\mathrm{br}, \mathrm{OH}), 1720(\mathrm{br}, \mathrm{C}=\mathrm{O}) \mathrm{cm}^{-1}$. Anal. Calcd. for $\mathrm{C}_{26} \mathrm{H}_{48} \mathrm{O}_{5}: \mathrm{C}, 70.87 ; \mathrm{H}$, 10.98. Found, C, 70.99; H, 11.08. 


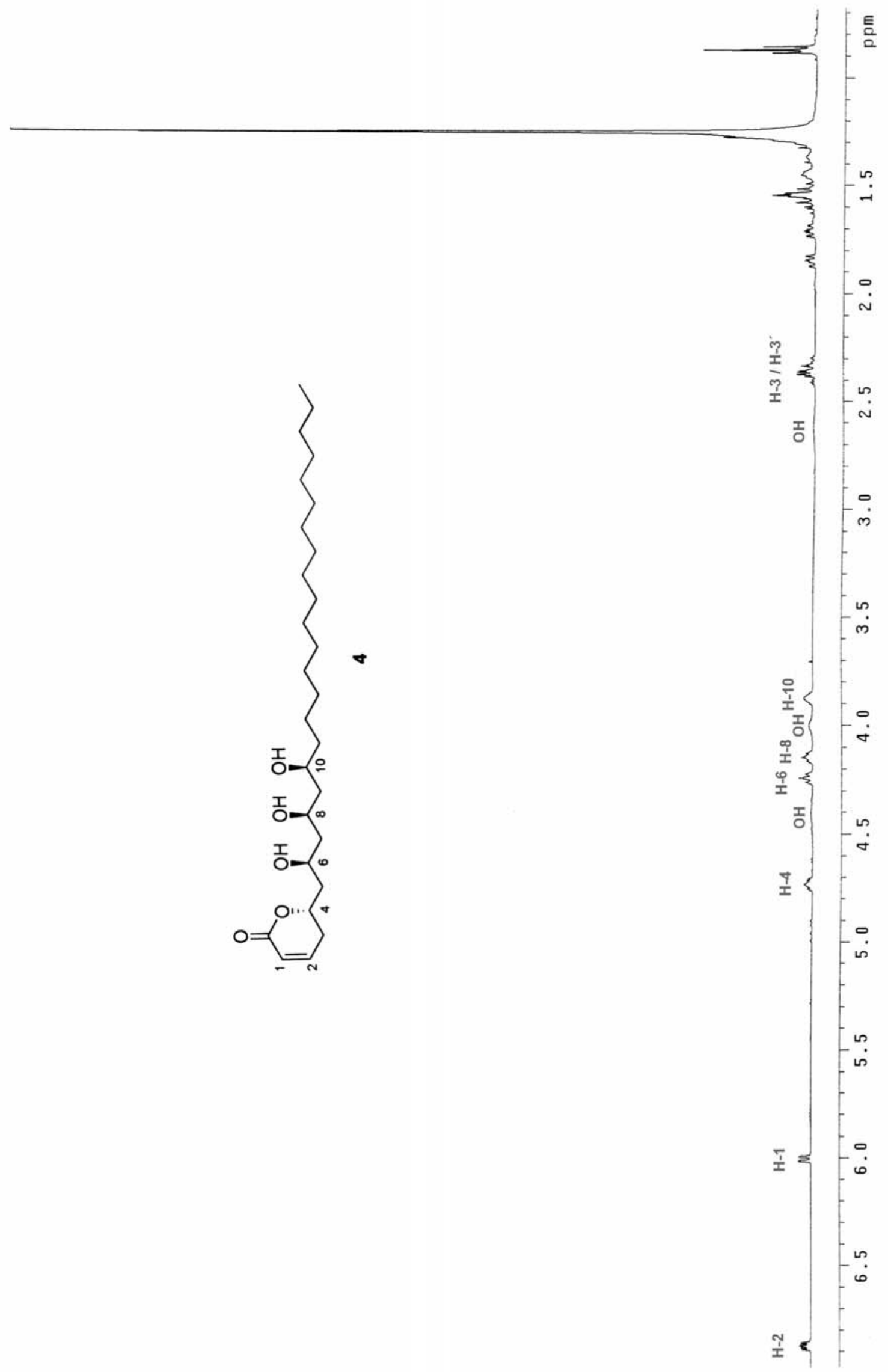




$$
A
$$




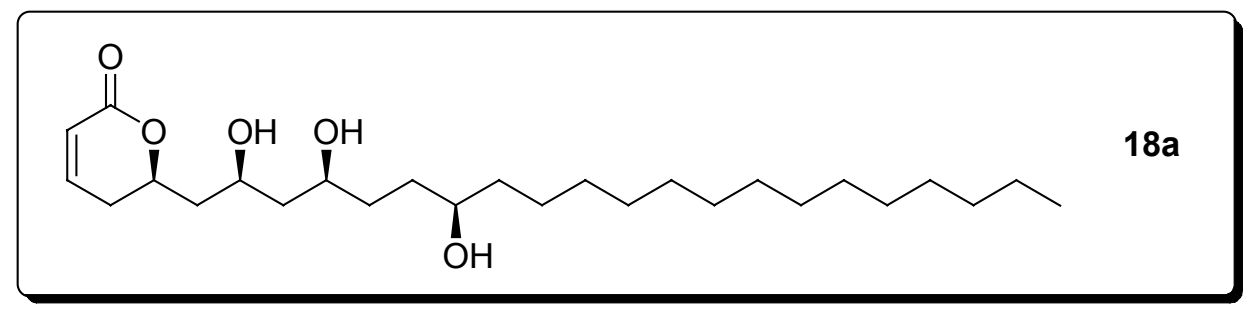

(R)-5,6-Dihydro-6-[(2S,4S,6R)-2,4,6-trihydroxyhenicosyl]pyran-2-one (18a): colorless solid, $\mathrm{mp}$ $80-81^{\circ} \mathrm{C}$ (from EtOAc-MeOH); $[\alpha]_{\mathrm{D}}+11.1\left(c\right.$ 0.7; MeOH). ${ }^{1} \mathrm{H}$ NMR $(500 \mathrm{MHz}) \delta 6.89$ (ddd, 9.7, 5.5, $3 \mathrm{~Hz}, 1 \mathrm{H}), 6.00$ (br d, $9.7 \mathrm{~Hz}, 1 \mathrm{H}), 4.67$ (sext, $6 \mathrm{~Hz}, 1 \mathrm{H}), 4.30$ (br s, OH, 1H), 4.11 (m, 1H), 3.90 (m, $1 \mathrm{H}), 3.60(\mathrm{~m}, 1 \mathrm{H}), 2.50-2.40(\mathrm{~m}, 2 \mathrm{H}), 2.04(\mathrm{dt}, \mathrm{J}=14,7.5 \mathrm{~Hz}, 1 \mathrm{H}), 1.78(\mathrm{dt}, \mathrm{J}=14,5.5 \mathrm{~Hz}, 1 \mathrm{H}), 1.70-$ 1.50 (br m, 4H), 1.50-1.40 (m, 2H), 1.35-1.20 (br m, 28H), 0.87 (t, $7 \mathrm{~Hz}, 3 \mathrm{H}) ;{ }^{13} \mathrm{C} \mathrm{NMR} \mathrm{(125} \mathrm{MHz)} \delta$ 164.3 (C), 145.5, 121.2, 76.2, 73.1, 72.4, $69.4(\mathrm{CH}), 43.1,42.3,37.9,35.2,33.8,31.9$, 29.7 (several overlapped peaks), 29.5, 29.4, 25.8, $22.7\left(\mathrm{CH}_{2}\right), 14.1\left(\mathrm{CH}_{3}\right)$. IR $v_{\max } 3330(\mathrm{br}, \mathrm{OH}), 1719(\mathrm{C}=\mathrm{O}) \mathrm{cm}^{-1}$. HR EIMS m/z (rel. int.) $441.3564\left(\mathrm{M}+\mathrm{H}^{+}, 1\right), 422\left(\mathrm{M}^{+}-\mathrm{H}_{2} \mathrm{O}, 2\right), 404\left(\mathrm{M}^{+}-2 \mathrm{H}_{2} \mathrm{O}, 6\right), 267$ (27), 225 (100), 141 (68). Calcd. for $\mathrm{C}_{26} \mathrm{H}_{49} \mathrm{O}_{5}$, 441.3580. Anal. Calcd. for $\mathrm{C}_{26} \mathrm{H}_{48} \mathrm{O}_{5}$ : C, 70.87; H, 10.98. Found, C, 71.02; H, 11.10. 


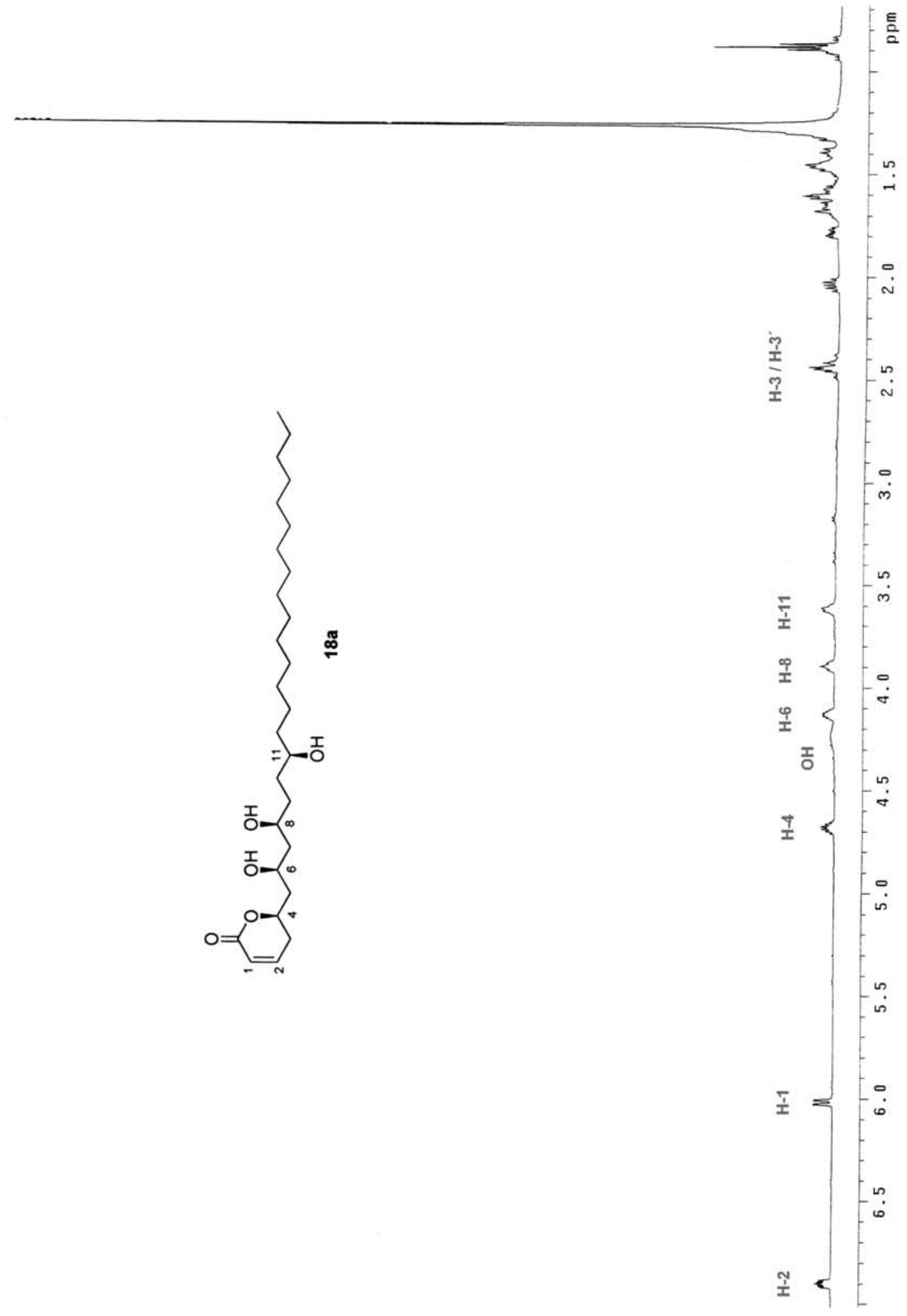




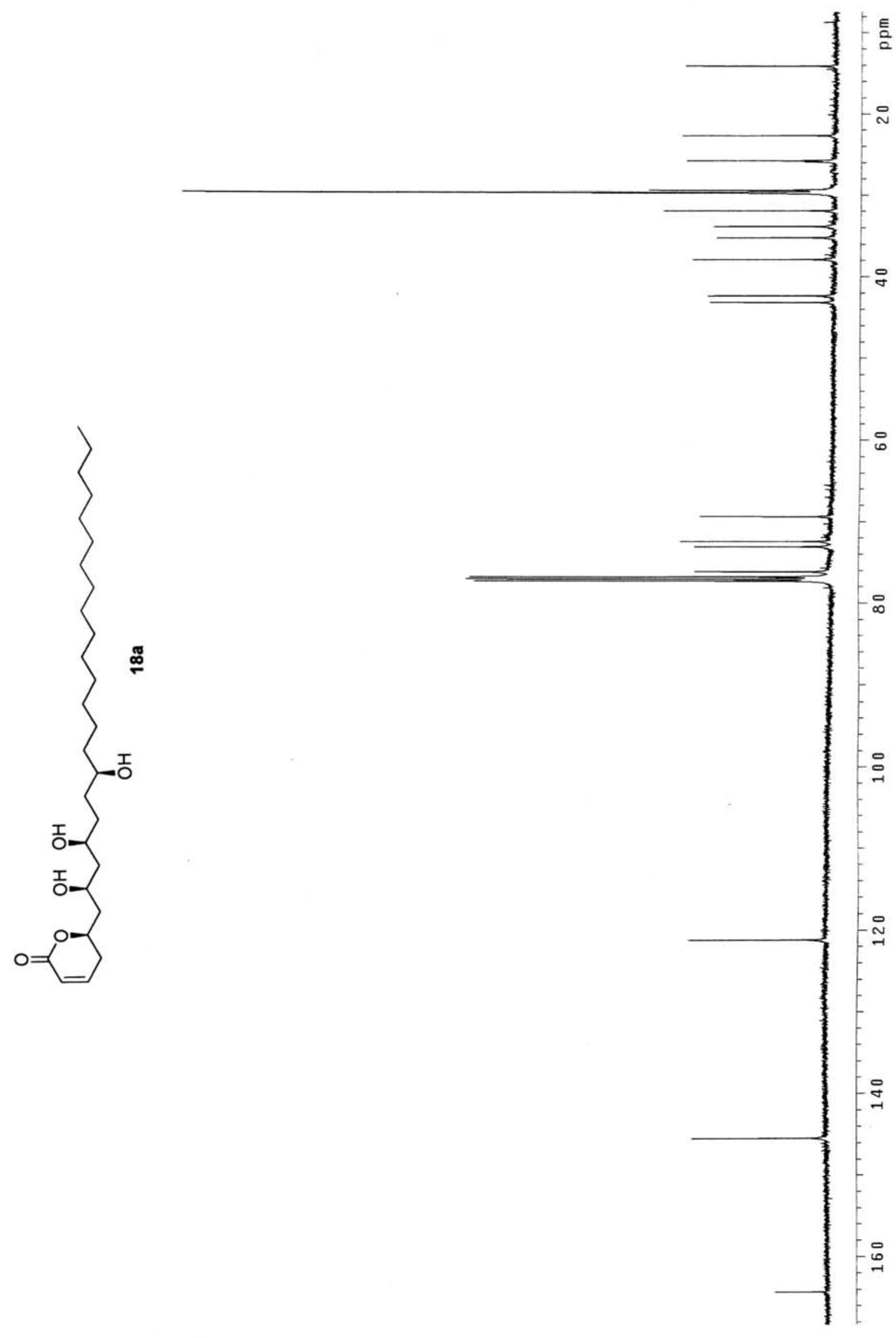




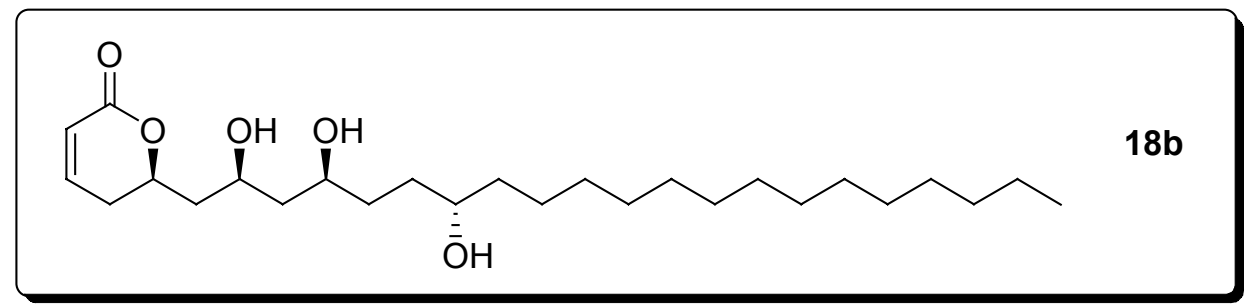

(R)-5,6-Dihydro-6-[(2S,4S,6S)-2,4,6-trihydroxyhenicosyl]pyran-2-one, passifloricin A (18b): colorless solid, mp $103-106^{\circ} \mathrm{C}$ (from EtOAc-MeOH), lit. for passifloricin A, mp $97^{\circ} \mathrm{C}$; $[\alpha]_{\mathrm{D}}+28.9(c$ 0.8; $\mathrm{MeOH})$, lit. for passifloricin $\mathrm{A},[\alpha]_{\mathrm{D}}+123.45(c 0.11 ; \mathrm{MeOH}) ;[\alpha]_{\mathrm{D}}+33.3\left(c 0.8 ; \mathrm{CHCl}_{3}\right)$ for $\mathbf{1 8 b}$; $[\alpha]_{\mathrm{D}}+34.1\left(\mathrm{c} 0.5 ; \mathrm{CHCl}_{3}\right)$ for a sample of natural passifloricin A. ${ }^{1} \mathrm{H} \mathrm{NMR}(500 \mathrm{MHz}) \delta 6.89(\mathrm{ddd}, 9.5$, 5.5, $3 \mathrm{~Hz}, 1 \mathrm{H}), 6.00$ (br d, $9.5 \mathrm{~Hz}, 1 \mathrm{H}), 4.66(\mathrm{sext}, 5.5 \mathrm{~Hz}, 1 \mathrm{H}), 4.30$ (br s, OH, 1H), $4.11(\mathrm{~m}, 1 \mathrm{H}), 3.95$ (m, 1H), $3.64(\mathrm{~m}, 1 \mathrm{H}), 2.50-2.40(\mathrm{~m}, 2 \mathrm{H}), 2.04(\mathrm{dt}, \mathrm{J}=14,7.5 \mathrm{~Hz}, 1 \mathrm{H}), 1.80(\mathrm{dt}, \mathrm{J}=14,5.5 \mathrm{~Hz}, 1 \mathrm{H})$, 1.70-1.50 (br m, 4H), 1.50-1.40 (m, 2H), 1.35-1.20 (br m, 28H), 0.87 (t, $7 \mathrm{~Hz}, 3 \mathrm{H}) ;{ }^{13} \mathrm{C}$ NMR $(125$ MHz) $\delta 164.4(\mathrm{C}), 145.5,121.2,76.2,72.5,71,9,69.5(\mathrm{CH}), 42.8,42.4,37.5,34.1,32.7,32.0,29.7$ (several overlapped peaks), 29.5, 29.4, 25.9, $22.7\left(\mathrm{CH}_{2}\right), 14.1\left(\mathrm{CH}_{3}\right) . \mathrm{IR} v_{\max } 3260$ (br, OH), 1715 $(\mathrm{C}=\mathrm{O}) \mathrm{cm}^{-1}$. HR EIMS $\mathrm{m} / z$ (rel. int.) $422.3411\left(\mathrm{M}^{+}-\mathrm{H}_{2} \mathrm{O}, 2\right), 404\left(\mathrm{M}^{+}-2 \mathrm{H}_{2} \mathrm{O}, 6\right), 267$ (32), 225 (100), 141 (66). Calcd. for $\mathrm{C}_{26} \mathrm{H}_{48} \mathrm{O}_{5}-\mathrm{H}_{2} \mathrm{O}, 422.3396$. The identity of natural and synthetic product was confirmed by the measurement of the NMR spectra of a mixture of both compounds. 


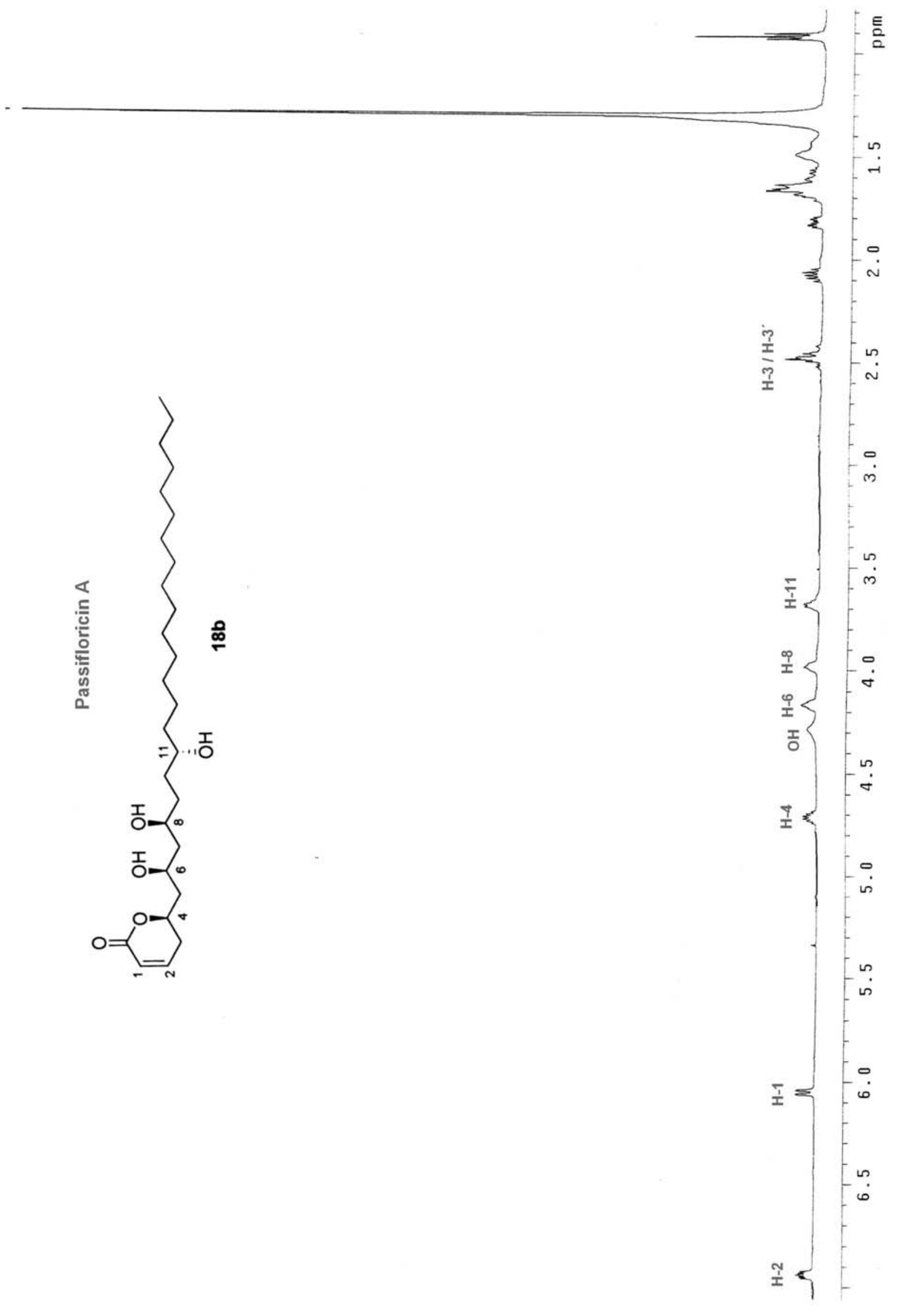




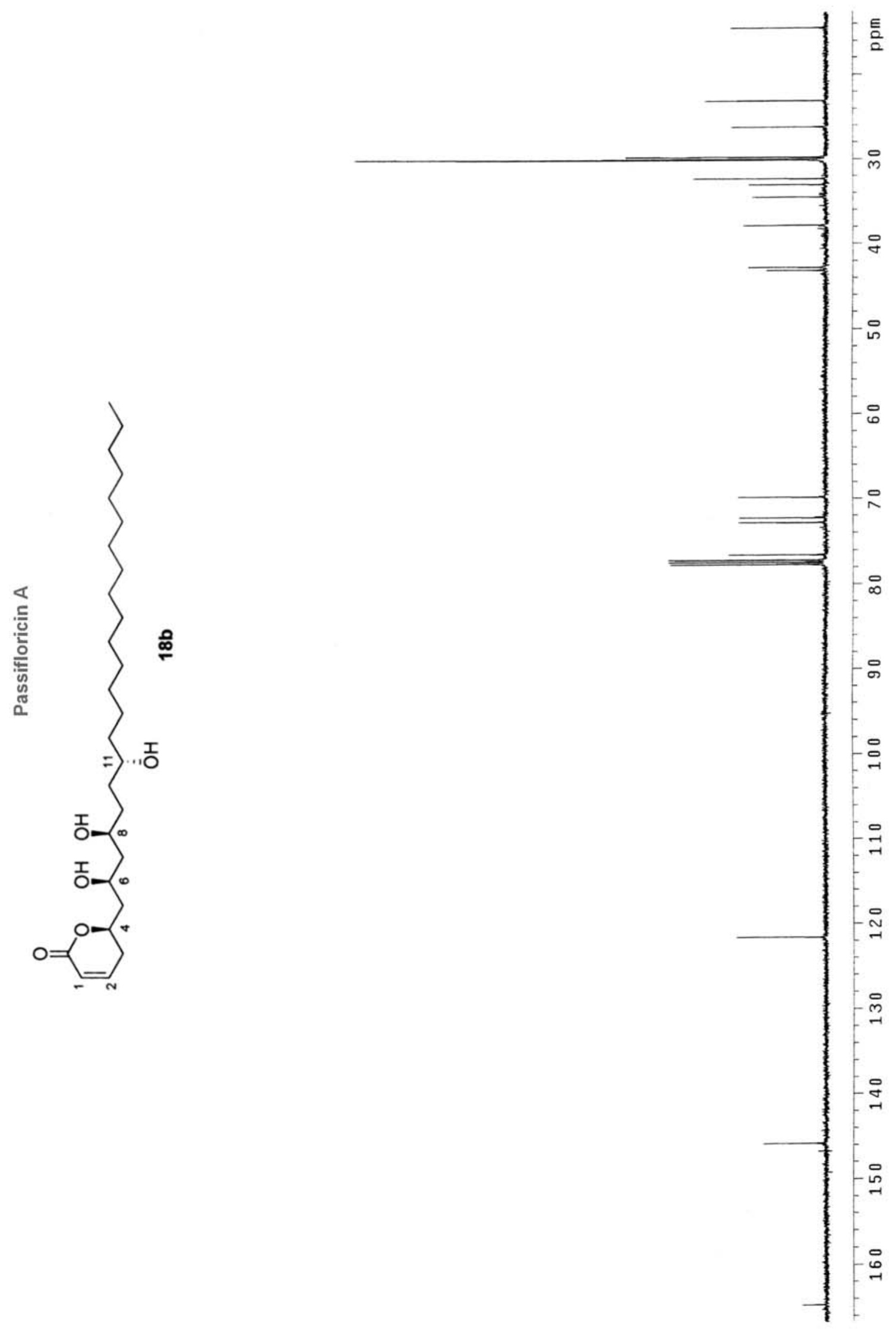




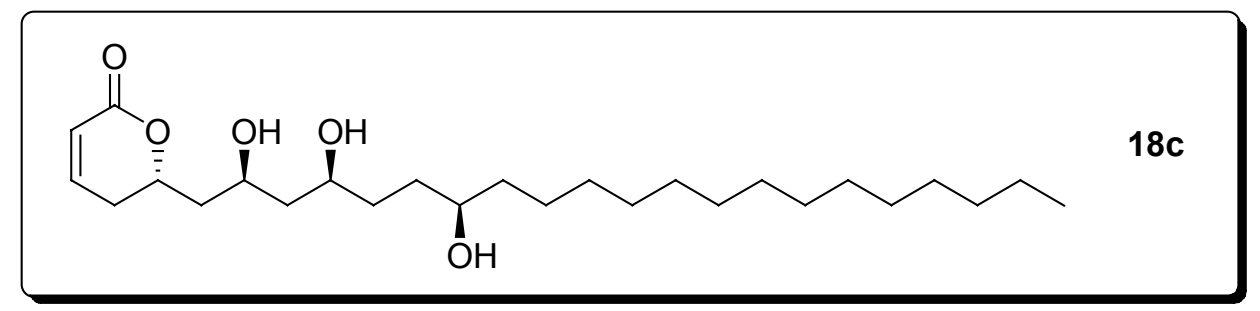

(S)-5,6-Dihydro-6-[(2S,4S,6R)-2,4,6-trihydroxyhenicosyl]pyran-2-one (18c): colorless solid, mp 82$83^{\circ} \mathrm{C}$ (from EtOAc-MeOH); $[\alpha]_{\mathrm{D}}-21.8$ (c 0.7; MeOH). ${ }^{1} \mathrm{H} \mathrm{NMR}(500 \mathrm{MHz}) \delta 6.89$ (ddd, 9.7, 5.6, 2.7 $\mathrm{Hz}, 1 \mathrm{H}), 6.01$ (dd, 9.7, $1 \mathrm{~Hz}, 1 \mathrm{H}), 4.76$ (m, 1H), 4.30 (br s, 1H, OH), 4.21 (m, 1H), 3.90 (m, 1H), 3.60 (m, 1H), 2.40-2.30 (m, 2H), 1.87 (ddd, 14, 9.5, $2.5 \mathrm{~Hz}, 1 \mathrm{H}), 1.80$ (br s, 1H, OH), 1.70-1.40 (br m, 6H), 1.35-1.20 (br m, 28H), 0.87 (t, $7 \mathrm{~Hz}, 3 \mathrm{H}) ;{ }^{13} \mathrm{C}$ NMR (125 MHz) $\delta 164.6$ (C), 145.5, 121.3, 74.9, 73.1, 72.5, $68.0(\mathrm{CH}), 43.7,43.2,37.9,35.3,33.9,31.9,30.0,29.7$ (several overlapped peaks), 29.4, 25.8, $22.7\left(\mathrm{CH}_{2}\right), 14.1\left(\mathrm{CH}_{3}\right)$. IR $v_{\max } 3330(\mathrm{br}, \mathrm{OH}), 1719(\mathrm{C}=\mathrm{O}) \mathrm{cm}^{-1}$. Anal. Calcd. for $\mathrm{C}_{26} \mathrm{H}_{48} \mathrm{O}_{5}: \mathrm{C}, 70.87$; H, 10.98. Found, C, 71.01; H, 10.90 . 


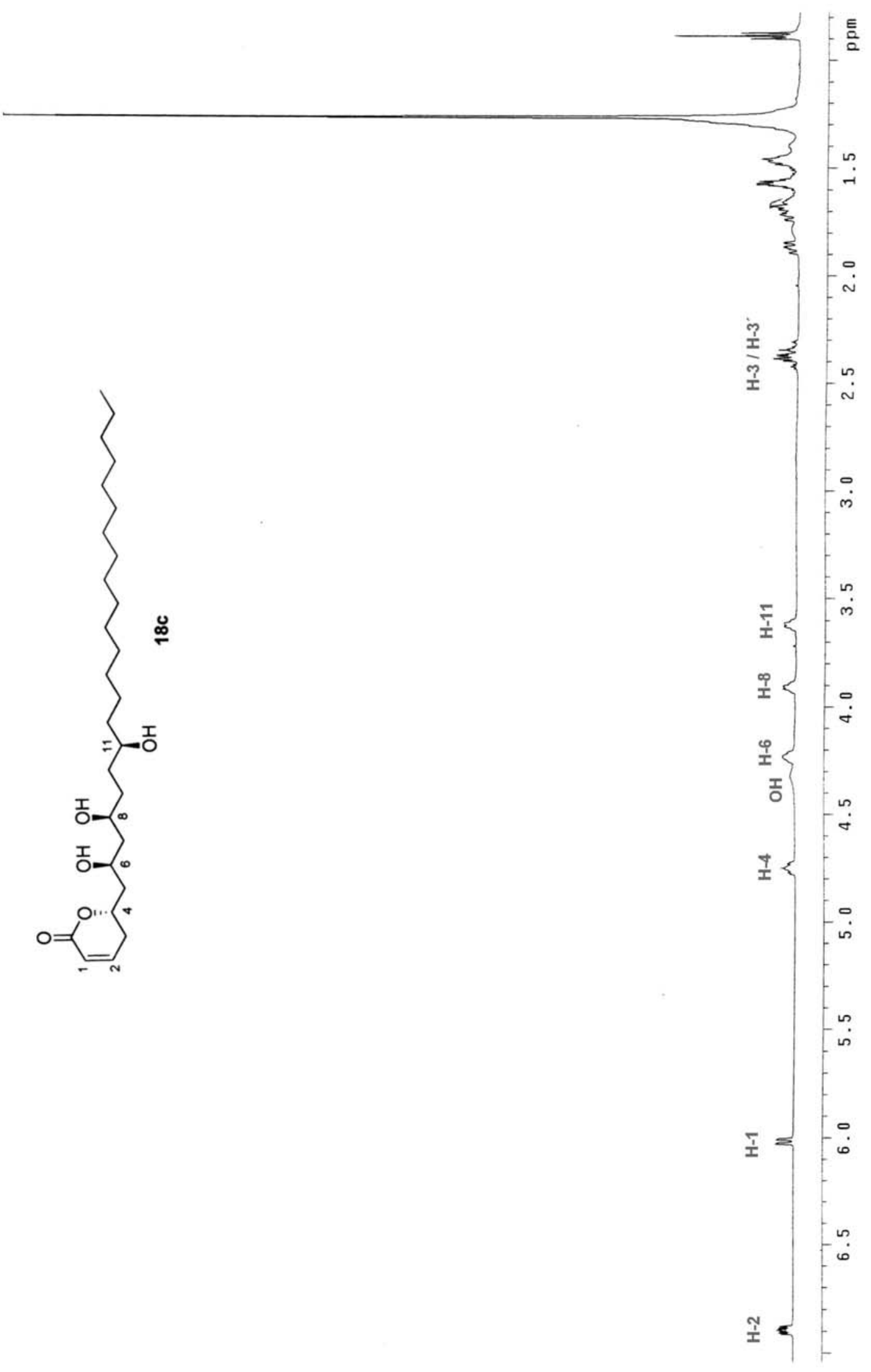




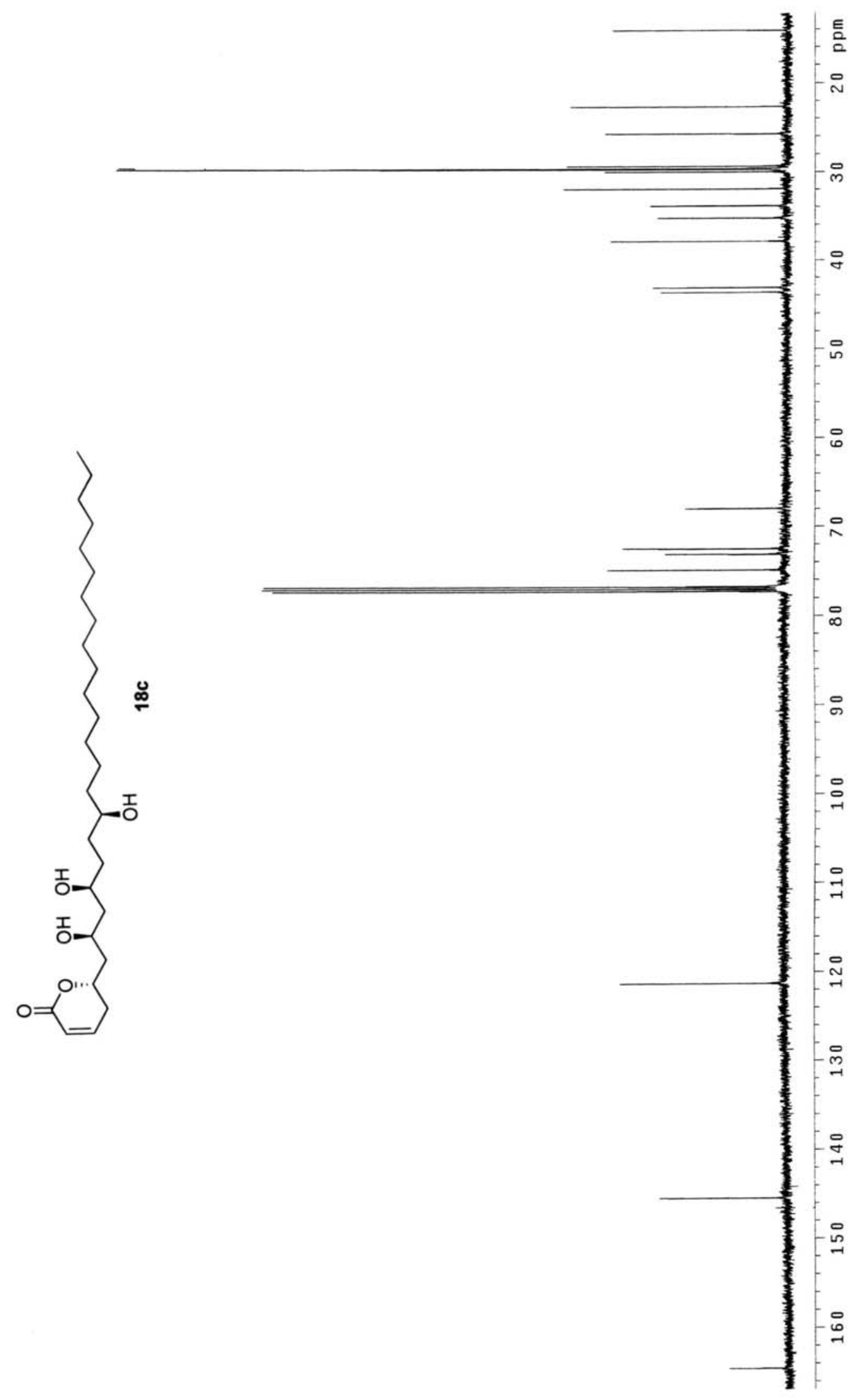




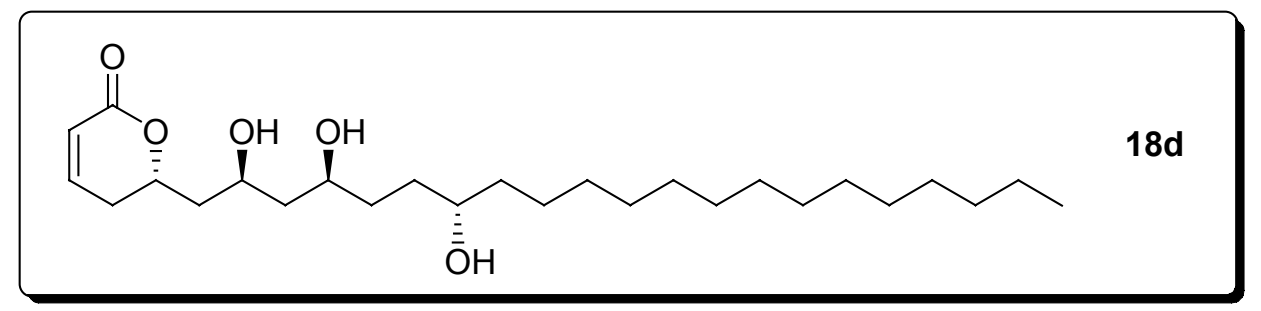

(S)-5,6-Dihydro-6-[(2S,4S,6S)-2,4,6-trihydroxyhenicosyl]pyran-2-one (18d): colorless solid, $\mathrm{mp}$ $118-119^{\circ} \mathrm{C}$ (from EtOAc-MeOH); $[\alpha]_{\mathrm{D}}+34.8\left(c\right.$ 0.45; $\left.\mathrm{CHCl}_{3}\right) .{ }^{1} \mathrm{H}$ NMR $(500 \mathrm{MHz}) \delta 6.89$ (ddd, 9.7 , 5.6, $2.7 \mathrm{~Hz}, 1 \mathrm{H}), 6.01(\mathrm{dd}, 9.7,1.5 \mathrm{~Hz}, 1 \mathrm{H}), 4.75(\mathrm{~m}, 1 \mathrm{H}), 4.21(\mathrm{~m}, 1 \mathrm{H}), 3.95(\mathrm{~m}, 1 \mathrm{H}), 3.64(\mathrm{~m}, 1 \mathrm{H})$, 2.40-2.30 (m, 2H), 1.86 (ddd, 14, 9.5, 2 Hz, 1H), 1.70 (ddd, 14, 10, $2.5 \mathrm{~Hz}, 1 \mathrm{H}), 1.65-1.40$ (br m, 6H), 1.35-1.20 (br m, 29H), 0.87 (t, $7 \mathrm{~Hz}, 3 \mathrm{H}) ;{ }^{13} \mathrm{C}$ NMR (125 MHz) $\delta 164.6$ (C), 145.5, 121.3, 74.9, 72.4, 71.9, $68.0(\mathrm{CH}), 43.3,43.2,37.4,34.1,32.6,31.9,30.0,29.7$ (several overlapped peaks), 29.4, 25.8, $22.7\left(\mathrm{CH}_{2}\right), 14.1\left(\mathrm{CH}_{3}\right)$. IR $v_{\max } 3330(\mathrm{br}, \mathrm{OH}), 1710$ (br, $\left.\mathrm{C}=\mathrm{O}\right) \mathrm{cm}^{-1}$. Anal. Calcd. for $\mathrm{C}_{26} \mathrm{H}_{48} \mathrm{O}_{5}: \mathrm{C}_{\text {, }}$ 70.87; H, 10.98. Found, C, 70.77; H, 11.11. 


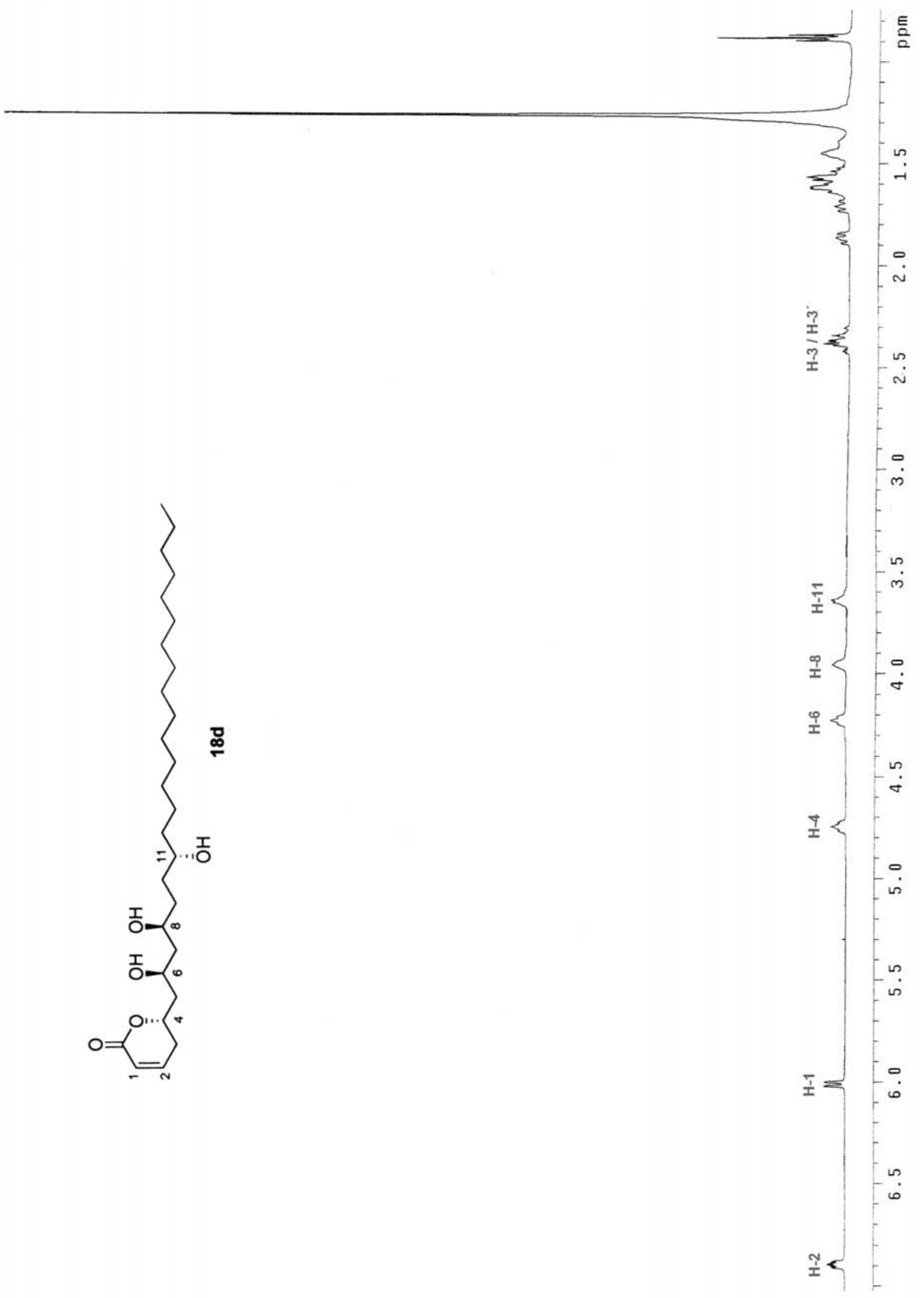




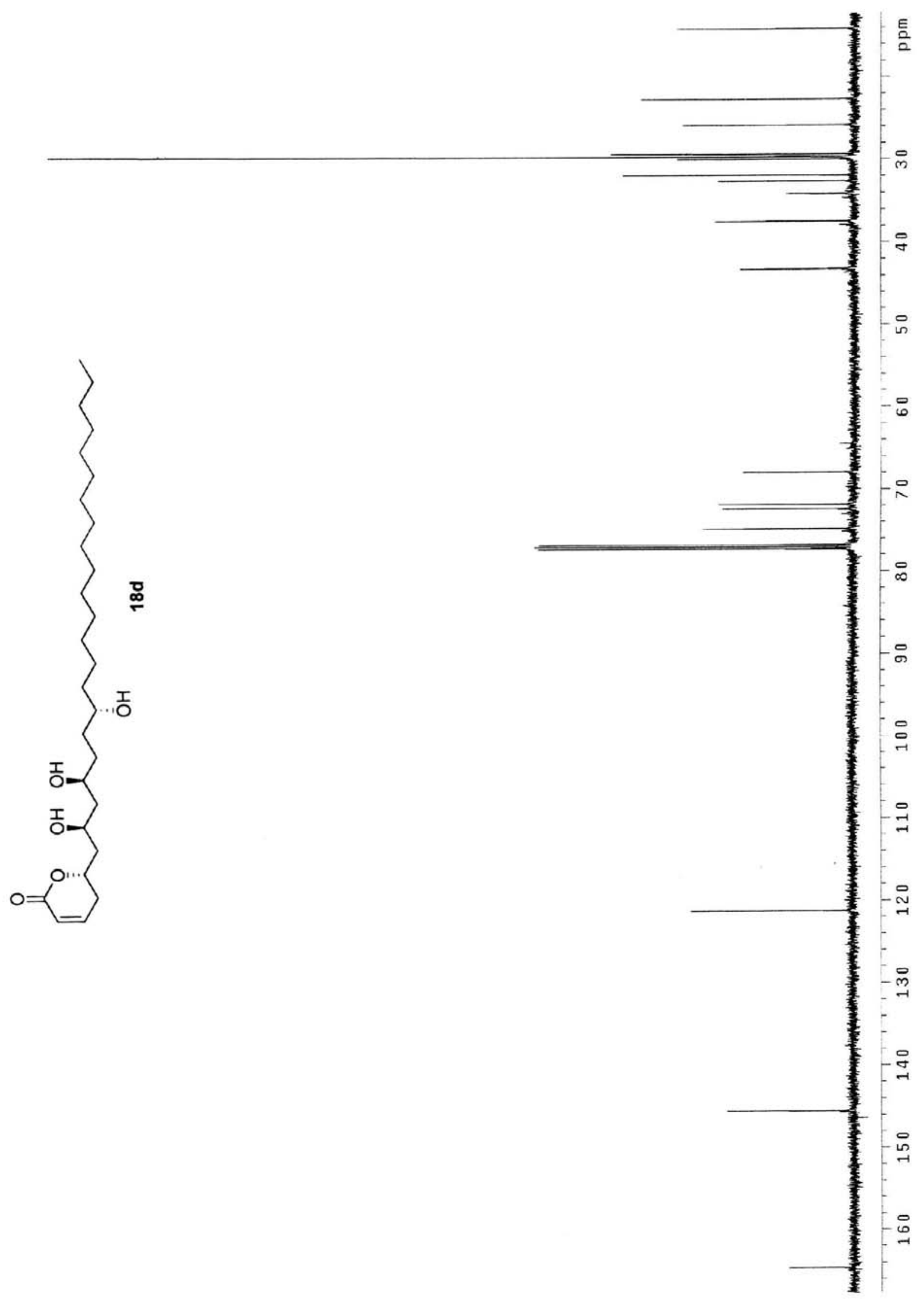

